\title{
Accelerated Prediction of Atomically Precise Cluster Structures Using On-the-fly Machine Learning
}

Yunzhe Wang, Shanping Liu, Peter Lile, Sam Norwood, Alberto Hernandez, Sukriti Manna, and Tim Mueller*

Department of Materials Science and Engineering, Johns Hopkins University, Baltimore, MD 21218

*Corresponding Author Email: tmueller@jhu.edu

KEYWORDS: nanocluster, genetic algorithm, machine learning interatomic potential, active learning, transferrable potential, structure search

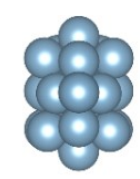

21

$\left(\mathrm{C}_{\mathrm{s}}\right)$

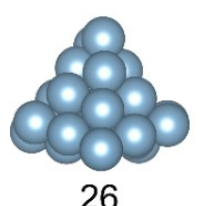

26

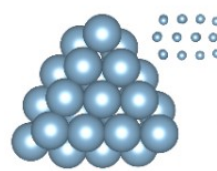

32

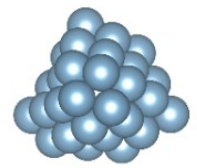

38

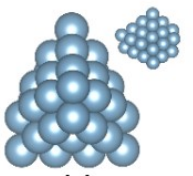

44

$\left(\mathrm{C}_{\mathrm{s}}\right)$

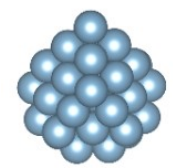

50

$\left(\mathrm{C}_{2}\right)$

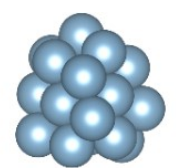

22_01
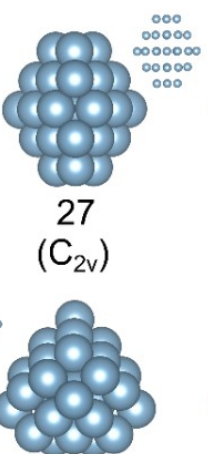

33

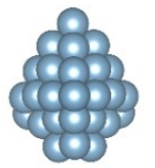

39

$\left(\mathrm{C}_{\mathrm{s}}\right)$
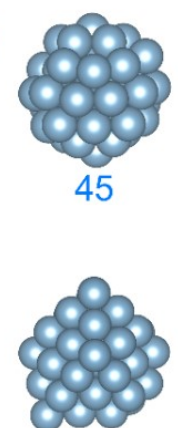

51

$\left(\mathrm{C}_{\mathrm{s}}\right)$

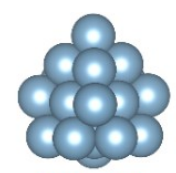

2202

$\left(\bar{C}_{\mathrm{s}}\right)$
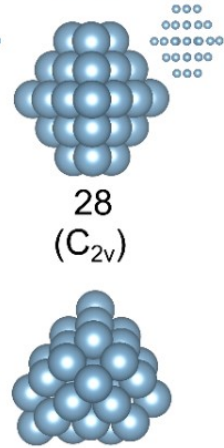

34

$\left(\mathrm{C}_{2}\right)$

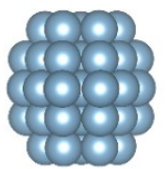

40

$\left(C_{2 v}\right)$

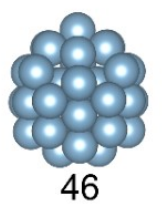

$\left(\mathrm{C}_{\mathrm{s}}\right)$

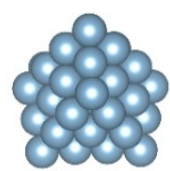

52

$\left(\mathrm{S}_{4}\right)$

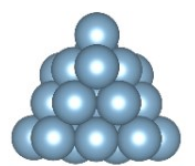

23

$\left(\mathrm{C}_{3 \mathrm{v}}\right)$

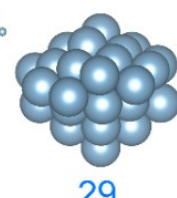

29

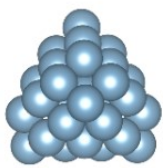

35

$\left(\mathrm{C}_{\mathrm{s}}\right)$

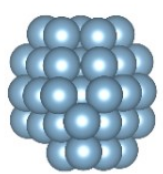

41

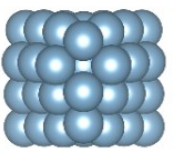

47

$\left(\mathrm{C}_{\mathrm{s}}\right)$

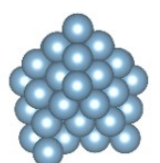

53

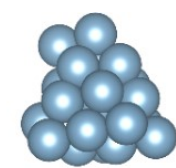

24

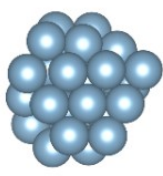

30

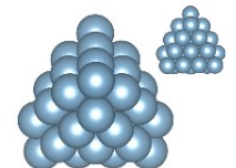

36

$\left(D_{2 d}\right)$

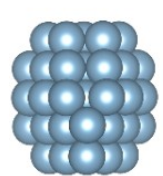

42

$\left(\mathrm{C}_{\mathrm{s}}\right)$

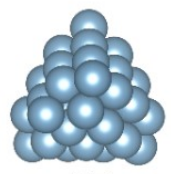

37

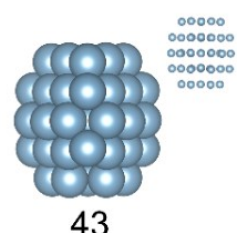

$\left(\mathrm{C}_{\mathrm{s}}\right)$
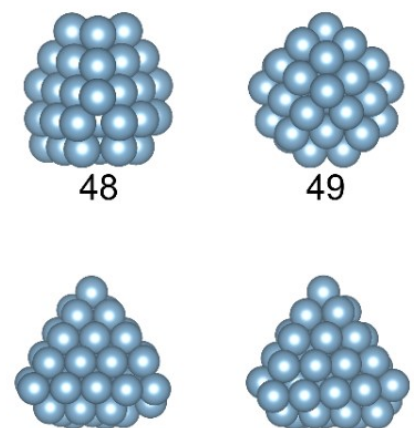

54

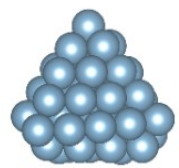

55 


\title{
Accelerated Prediction of Atomically Precise Cluster Structures Using On-the-fly Machine Learning
}

Yunzhe Wang, Shanping Liu, Peter Lile, Sam Norwood, Alberto Hernandez, Sukriti Manna, and Tim Mueller*

Department of Materials Science and Engineering, Johns Hopkins University, Baltimore, MD 21218

*Corresponding Author Email: tmueller@jhu.edu

KEYWORDS: nanocluster, genetic algorithm, machine learning interatomic potential, active learning, transferrable potential, structure search

\begin{abstract}
The chemical and structural properties of atomically precise nanoclusters are of great interest in numerous applications, but predicting the stable structures of clusters can be computationally expensive. In this work, we present a procedure for rapidly predicting low-energy structures of nanoclusters by combining a genetic algorithm with interatomic potentials actively learned on-the-fly. Applying this approach to aluminum clusters with 21 to 55 atoms, we have identified structures with lower energy than any reported in the literature for 25 out of the 35 sizes. Our benchmarks indicate that the active learning procedure accelerated the average search speed by more than an order of magnitude relative to genetic algorithm searches using only density functional calculations. This work demonstrates a feasible way to systematically discover stable structures for large nanoclusters and provides insights into the transferability of machine-learned interatomic potentials for nanoclusters.
\end{abstract}

\section{INTRODUCTION}

Nanoclusters have drawn much attention due to their special physical and chemical properties ${ }^{1,2}$ which are distinct from molecules or bulk crystal materials. These properties make them useful in diverse research fields including catalysis, ${ }^{3-6}$ chemical sensing, ${ }^{7}$ fluorescence,${ }^{8,9}$ and medicine. ${ }^{10}$ The unique properties of nanoclusters are largely the consequence of distinct size-dependent atomic structures, quantum finite size effects, and very large surface-to-volume ratios. ${ }^{11,12}$ These properties are generally not smooth functions of cluster sizes and can fluctuate with the addition or removal of a single atom.

Computational screening is a promising way to identify nanoclusters with desirable properties, but to predict the properties of a nanocluster from first principles it is necessary to first identify the low-energy atomic structures of the cluster. Many optimization methods have been proposed to perform global structure searches for nanoclusters, including the basin hopping method, ${ }^{13,14}$ unbiased random sampling ${ }^{15}$, particle swarm optimization, ${ }^{16,17}$ simulated annealing ${ }^{18,19}$ and genetic

algorithms (GA). ${ }^{20-24}$ Each of these methods involves the evaluation of the energies of a large number of candidate structures, which makes it critically important to evaluate structure energies with a method that is both fast and sufficiently accurate to distinguish between competing 
structures. Density functional theory (DFT) provides a high level of accuracy, but its speed and scalability typically limit the search to nanoclusters of sizes up to only several dozen atoms. ${ }^{25-28}$ Classical interatomic potentials, which typically have simple functional forms derived from fundamental physics, are several orders of magnitude faster than DFT and have been used to search for ground state structures with up to a few hundred atoms. ${ }^{13,29,30}$ However, classical interatomic potentials often lack the accuracy required to resolve the energy differences between competing candidate structures, especially for the low-lying local minima on the potential energy surface (PES) that are often only tens of meV apart. ${ }^{20,31}$

In recent years, an alternative type of interatomic potential has emerged in the form of machinelearned interatomic potentials (MLIPs) ${ }^{32-36}$ which are parameterized by fitting to a set of training data. Examples of MLIPs are neural network potentials, ${ }^{37-39}$ Gaussian approximation potentials (GAP), ${ }^{40-42}$ spectral neighbor analysis potentials (SNAP), ${ }^{43,44}$ moment tensor potentials (MTP), ${ }^{45-}$ ${ }^{47}$ the Atomic Cluster Expansion (ACE), ${ }^{48}$ and potentials found through symbolic regression. ${ }^{49}$ Although they may be slower than traditional interatomic potentials by an order of magnitude or more, MLIPs are generally more accurate and are still orders of magnitude faster than ab initio calculations. $^{50}$

Here we demonstrate how machine-learned interatomic potentials, in the form of moment tensor potentials, can be used to significantly accelerate a genetic algorithm search for low-energy cluster structures. Moment tensor potentials have been shown to have a good balance between accuracy and speed for bulk materials, ${ }^{50}$ and we demonstrate that they also work well for small atomic clusters. One of the challenges in using machine-learned interatomic potentials to search for ground state structures is that because the ground state is unknown, it is difficult to ensure that the potential is constructed in a way that will yield accurate ground state energies. To address this challenge we use active learning, in which the potential is trained adaptively with new data generated during the search. The idea of refining an interatomic potential model during the structural search was introduced by Hartke using classical interatomic potentials and proved to be capable of discovering new low-energy silicon clusters. ${ }^{51}$ Recently, similar strategies have also been successfully applied using MLIPs for global optimization of bulk crystalline materials ${ }^{52-55}$ and nanoclusters. ${ }^{56,57}$

We demonstrate our approach by searching for low-energy structures for aluminum clusters of between 21 and 55 atoms. Using this approach, we have discovered new cluster structures for 25 out of the 35 sizes that are at least $1 \mathrm{meV} /$ atom lower in DFT-calculated energy than the lowestenergy structures we have found in the literature..$^{28,29,58-60}$ New low-energy structures for an additional two sizes were discovered by DFT-only genetic algorithms used for benchmarking. Our approach, described in detail below, provides a template that can be used to significantly accelerate the computational design of atomic clusters, and paves the way for determining atomic structures of large nanoclusters. 


\section{RESULTS}

\section{Hyperparameter selection for Moment Tensor Potentials}

Existing benchmarks of MTP on bulk crystalline structures ${ }^{50,61}$ give generally good sets of parameters for training reliable MTP, but little information is available on good parameters for training clusters. To identify a good set of parameters for our calculations we used Al clusters with 24 atoms as a model system and tested various combinations of hyperparameters, including potential complexity (defined by the parameter $\left.l e v_{\max }\right),{ }^{47}$ the amount of training data, and the weight for force components (the "force weight") relative to the weight for energies. One quarter of the structures were randomly selected for validation, with the rest used to train the potential. Additional details about the construction of this dataset are provide in the Methods section and Section 2.1 of the Supplementary Information.

For a fixed training set, both energy and force errors decrease steadily as increasingly complex potentials are used (Figure 1b). However, such a gain is at the expense of an exponential growth in training costs (Figure 1c). Additional analysis of other combinations of hyperparameters (Figure S4 to Figure S7 of Supplementary Information) shows similar trends as in Figure 1. To balance accuracy and training costs we used and $l e v_{\max }=14$ and a force weight that was $1 / 1000$ that of the energy weight for all subsequent active learning genetic algorithm (GA_AL) runs. Using these parameters, we found that force and energy errors plateau after the training set exceeded about 1000 structures (Figure 1d). 

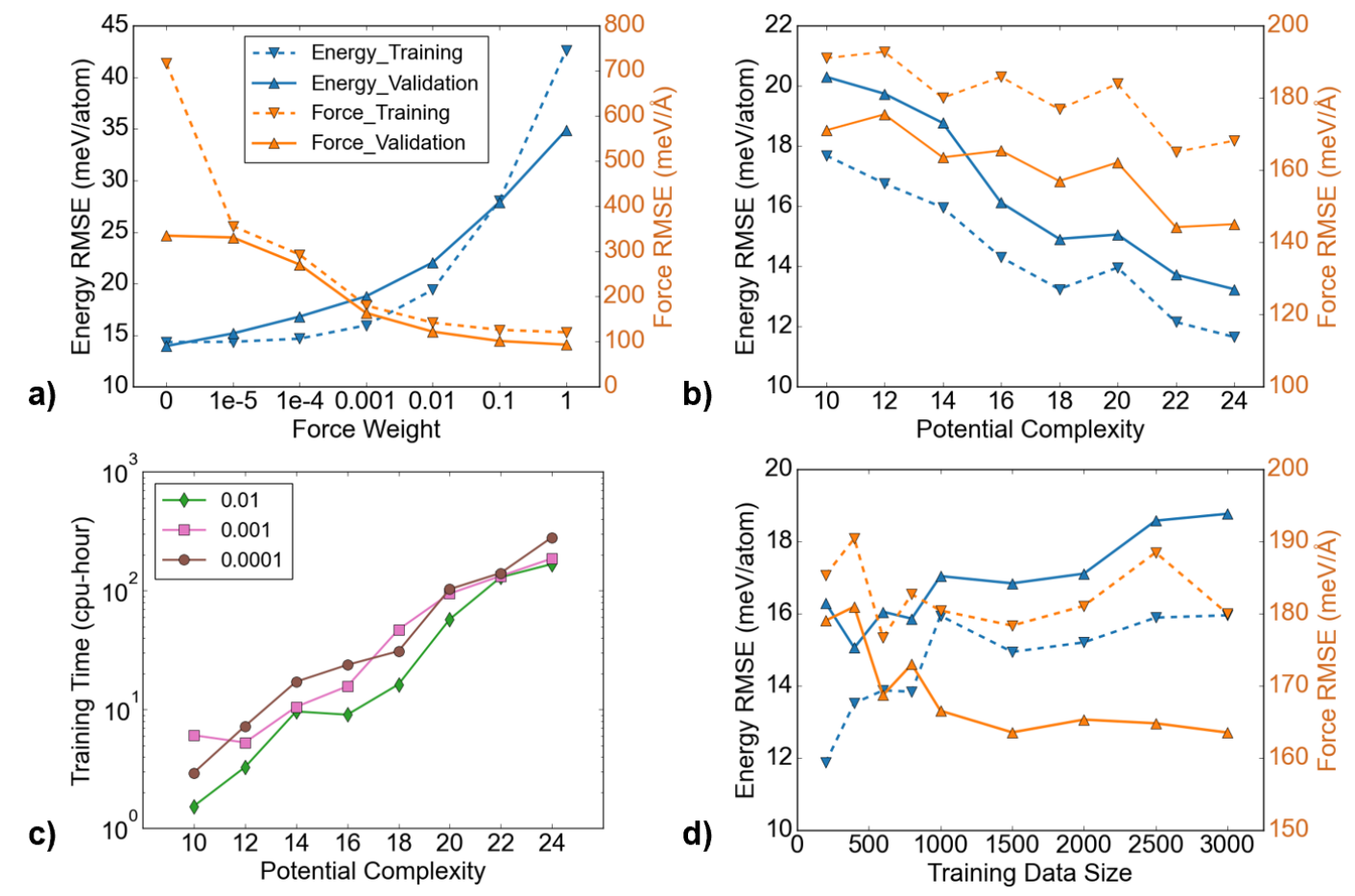

Figure 1. Benchmarks of hyperparameters for training MTP potentials. Training (dashed lines) and validation (solid lines) root-mean-squared errors for both energies and force components are plotted against a) force weights relative to the energy weight, b) potential complexity $l v_{\max }$, and d) the number of structures in the training set. a), b) and d) share the same legend as shown in a). Training costs of potentials with different relative force weights and complexities are shown

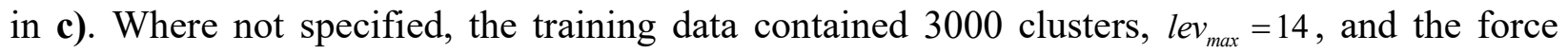
weight was $1 / 1000^{\text {th }}$ that of the energy weight.

\section{Prediction of cluster structures for Al clusters with $21-40$ atoms}

We evaluated our approach by predicting structures of aluminum nanoclusters with 21 to 40 atoms (Figure 2). The performance of the GA_AL algorithm was evaluated by comparing it with a genetic algorithm that used only DFT to calculate energies (GA_DFT), where both algorithms were run for the same amount of computing time. For our initial evaluation, GA_AL was initialized with untrained potentials and a new potential was trained at each cluster size. Details of how we performed the comparison are provided in the Methods section.

For 8 cluster sizes $(21,23,24,26,28,33,35$ and 36), mostly among the smaller clusters, GA_AL and GA_DFT found essentially the same lowest-energy clusters, with similarity scores, a measure of geometrical differences, below 0.3 (see the Methods section). For 10 out of the 20 sizes $(25,27$, $30-32,34,37-40)$, GA_AL found clusters that were lower in energy than those found by GA_DFT by at least $1 \mathrm{meV} /$ atom, with an average energy difference of $-5.06 \mathrm{meV} /$ atom (or $169.64 \mathrm{meV} / \mathrm{cluster}$ ). For clusters of 22 atoms, GA_AL identified a distinct cluster with a calculated energy within $0.1 \mathrm{meV} /$ atom of the lowest-energy cluster identified by GA_DFT. The 
lowest-energy 33-atom cluster found by GA_AL is $1.47 \mathrm{meV} /$ atom lower in energy than the one found by GA_DFT, but it is structurally similar based on both the similarity score and visual inspection (see Figure S14 of Supplementary Information). Therefore it is not counted as a new lowest-energy cluster. There was only one cluster size (29 atoms), for which GA_DFT found a distinct cluster with lower energy than that found by GA_AL. For this size the cluster found by GA_DFT was lower in energy by $3.61 \mathrm{meV} /$ atom (104.69 meV/cluster). On average, the energies of structures found by GA_AL are lower by $2.43 \mathrm{meV} /$ atom $(82.27 \mathrm{meV} / \mathrm{cluster})$.

To quantify how much more quickly the GA_AL approach finds low-energy structures, we define the "acceleration ratio" as the ratio of time it took GA_DFT to find its lowest-energy structure divided by the time it took GA_AL to find a structure with at least as low of the energy. In the case of size 29, GA_AL failed to discover better or equivalent configurations, so the ratio is set to 0 . The average acceleration ratio across all 20 sizes is 2.29 (Figure 2b). GA_DFT often did not find a cluster with energy as low as that found by GA_AL (Figure 2b), suggesting that if the acceleration ratio were based on the time required to find the lowest-energy structure it would be larger. Additional data illustrating the acceleration of GA_AL relative to GA_DFT are provided in Figure S11 and Figure S12 of the Supplementary Information. 

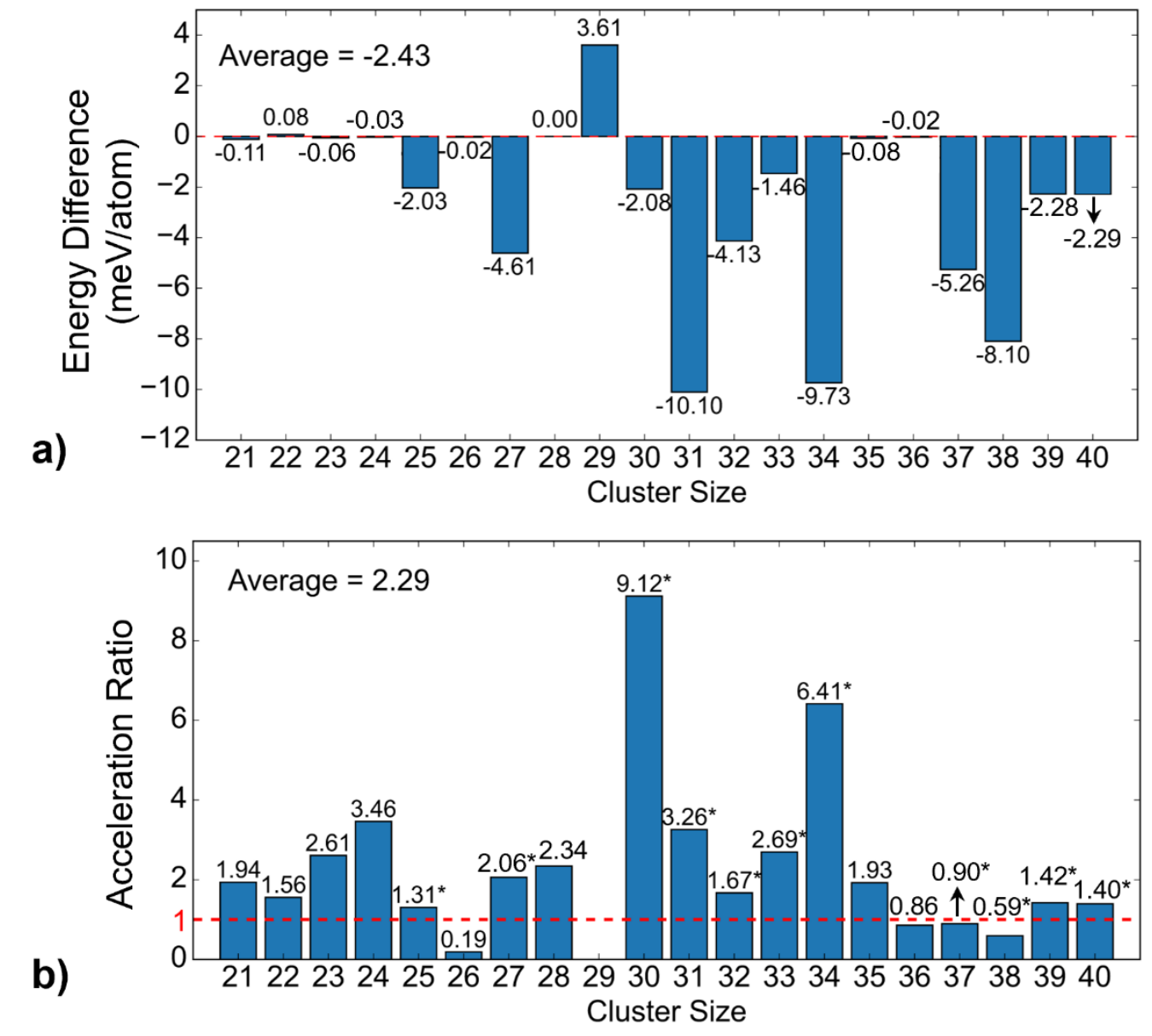

Figure 2. Performance benchmarks between GA_AL initialized with an untrained potential and GA_DFT. a) DFT-calculated energy differences between the lowest-energy structures found in GA_AL and GA_DFT. Negative values indicate GA_AL discovered structures with lower energies. b) Acceleration ratios of GA_AL relative to GA_DFT. The sizes for which GA_AL discovered better structures are marked with *, suggesting that they could be larger if GA_DFT were allowed to run for longer time.

\section{Size-transferable interatomic potentials for nanoclusters}

The results presented in the previous section were obtained by training a new potential at every cluster size, as there is a risk that a potential trained on one cluster size might not work well for clusters of another size due to the fact that the properties of atomic clusters can change discontinuously with the number of atoms in the cluster. However using a potential trained at one size to find structures of a different size could significantly speed up the structure search by reducing the total amount of training data that must be generated. In particular, using potentials trained with smaller clusters to predict the structures of larger clusters can have significant performance advantages, as the cost of generating training data using DFT typically scales as approximately the cube of the number of valence electrons in the cluster. ${ }^{62}$ 
We examined how accurately potentials trained on clusters of a range of small sizes are able to predict the energies of clusters with larger sizes. Training data was separated into a group of 3000 clusters with an even number of atoms $(22,26,30,34$, and 38) and another group of 3000 clusters with an odd number of atoms $(21,25,29,33$, and 37), as DFT calculations indicate that even-sized clusters and odd-sized clusters have distinct ground state magnetic moments (see Section 4 of the Supplementary Information). A third training set was created by combining the even and odd sets. The validation sets were composed of about 3000 clusters for each cluster size between 50-55 atoms. Details of the construction of the training and validation sets can be found in the Methods section.

All three mixed-size potentials predicted energies of the large clusters with validation errors $(\sim 10$ meV/atom) comparable to training errors (Figure $3 \mathrm{a}$ and Figure $3 \mathrm{~b}$ ). The errors in the predicted forces $(\sim 165 \mathrm{meV} / \AA)$ were slightly worse than the fitting errors. These errors are similar to the training and validation errors achieved when all of the training and validation data consisted of clusters of 24 atoms (Figure 1). Mixing training data with different magnetic moments did not have a significant adverse effect on model predictions (Figure 3 ). The potential trained on clusters with an odd number of atoms has slightly smaller prediction errors for both force and energy than potential trained on clusters with an even number of atoms regardless of whether the validation set contains even-sized or odd-sized clusters. The potential trained with both even and odd clusters has larger energy training errors than both of the even and odd potentials, but energy validation errors between the validation errors for even and odd potentials. For forces, the potential trained with both even and odd clusters has the lowest training and validation errors in all cases.

For comparison, we evaluated the ability of potentials trained on clusters of a single size. For each size, the training data consisted of 3000 dissimilar clusters, with exception of clusters with 21 atoms (the smallest size) for which our training set only had 2136 clusters after removing structurally similar clusters (see Methods section). For potentials trained on clusters of a single size, training and validation errors were similar for forces, and potentials trained on a single size may predict forces with significantly lower errors than the potentials trained on a mixed set of sizes. However validation errors for energies are notably worse than the training errors, especially for potentials trained on small clusters (Figure $3 \mathrm{a}$ and Figure $3 \mathrm{~b}$ ). The accuracy strongly depends on the size of the clusters in the training set, with larger sizes having the lowest errors. This suggests that quantum finite-size effects may be particularly pronounced for clusters with fewer than about 30 atoms, limiting the extent to which potential models trained at these sizes can be transferred to larger sizes. The training algorithm may also have a difficult time determining how the undercoordination of surface atoms in a cluster affects its energy when all clusters in the training set have approximately the same surface area. In contrast, training sets with a mixture of cluster sizes provide more information on how the energy is affected by the cluster surface area, which may improve the prediction accuracy for clusters of varying size.

Because of the particular importance of identifying the structures of low-energy clusters, we evaluated the potentials on the lowest-energy structures we found or collected from the literature with 50 to 55 atoms. The diversity of the training sets with mixed cluster sizes proved beneficial for identifying low-energy clusters, as the MTP extrapolation grade (see Methods section) for the 
lowest-energy clusters in the validation set is less than one, suggesting interpolation, with respect to the training sets of odd-sized and even-sized clusters. On the other hand, the low-energy structures had an extrapolation grade above 1, suggesting extrapolation, with respect to the training sets of single-sized clusters. Accordingly, the mixed-size potentials had much lower energy errors than the single-size ones (see Figure S14 of the Supplementary Information).

a)

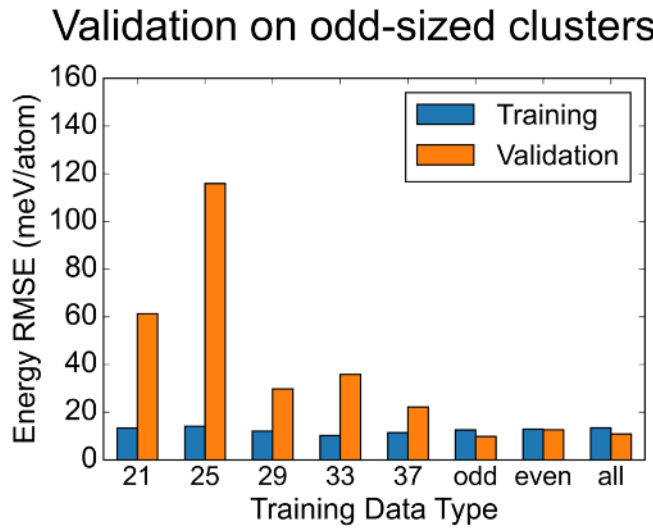

c)

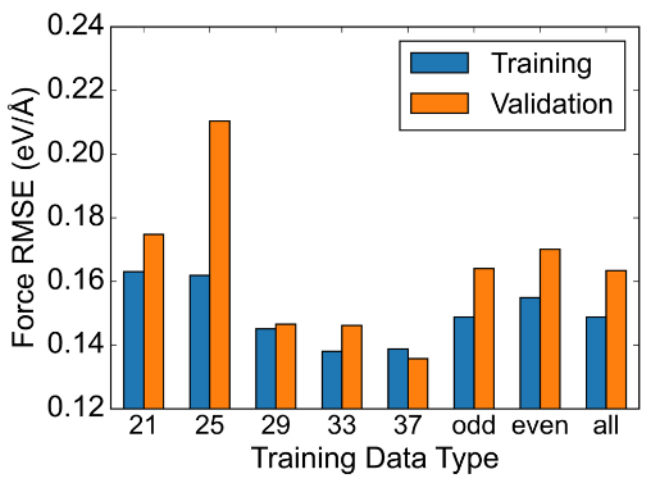

b)
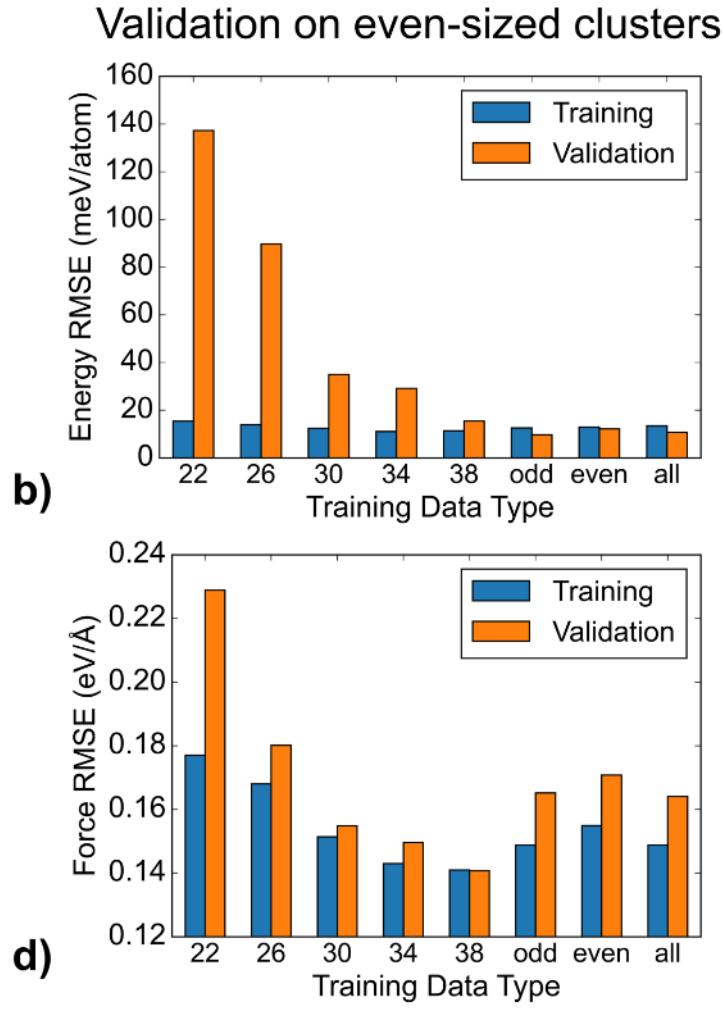

Figure 3. Training and validation root-mean-square errors in energy and force components for different potentials. The validation data consisted of clusters with 50-55 atoms. Plots in the left column show a) energy and c) force errors for potentials validated on odd-sized clusters, while the right column displays b) energy and d) force errors for potentials validated on even-sized structures. Potentials are labeled by the type of training data used to generate them. Numeric labels represent single-size potentials whose training sets contain exclusively clusters of the labelled sizes. The labels "odd", "even" and "all" represent potentials whose training sets are made up by clusters with odd, even, and mixture of odd and even number of atoms respectively.

\section{Prediction of structures for Al clusters with 41-55 atoms}

To identify low-energy structures with 41-55 atoms, we used the mixed-size potentials trained on clusters with an odd or even number of atoms to initialize GA_AL searches for clusters with an odd or even number of atoms respectively. Initializing the GA_AL algorithm with a pre-trained potential demonstrated significant performance advantages, with an average acceleration ratio of 12.1 compared to GA_DFT. For sizes 45 and 54, the acceleration ratio was set to 0 since they failed to discover better or equivalent configurations as GA_DFT. 
The sizable increase of acceleration ratios for GA_AL with pre-trained potentials can be credited to a significant reduction in the number of times DFT is called for learning on the fly in the early stages of the search. For GA_AL runs initialized with an untrained potential, clusters in the early stages of the runs tend to have high energies (Figure 4a), so training steps in the early stages are sampling a relatively high-energy region of configuration space. For GA_AL runs initialized with well-trained potentials, computational resources are more efficiently spent exploring the lowenergy configurations.
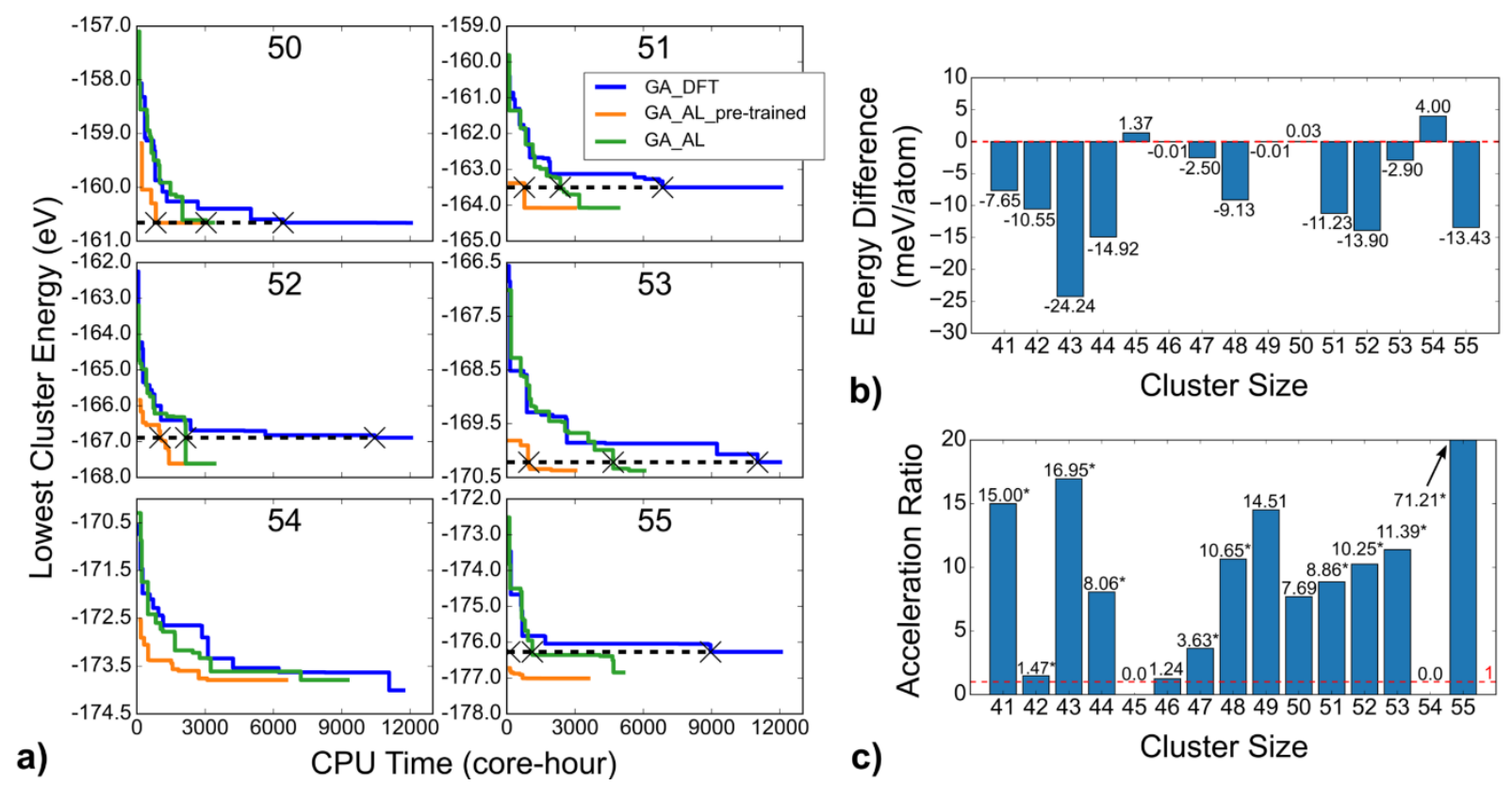

b)

Cluster Size

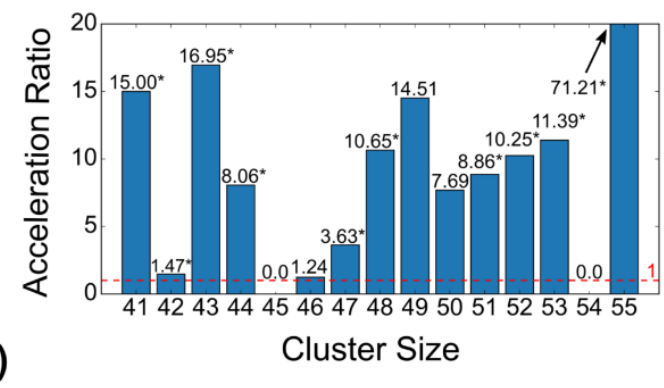

Figure 4. Performance benchmark of GA_AL against GA_DFT on clusters with 41 to 55 atoms. a) Energy evolution plots for GA_AL initialized with untrained potentials (green), GA_AL initialized with mixed-size potentials (orange) and GA_DFT (blue), for clusters with 50 to 55 atoms. The energy levels used to calculate acceleration ratios are marked by dashed lines. The CPU times used to compute acceleration ratios are marked by crosses. b) Energy differences between the lowest energies of GA_AL and GA_DFT at the end of simulations. c) Acceleration ratios of GA_AL relative to GA_DFT. The sizes for which GA_AL successfully discovered configurations with lower energy than the lowest of GA_DFT are marked by *.

\section{Literature comparison}

We examine the quality of the ground state configurations discovered using GA_AL for clusters of 21-55 atoms by comparing them with lowest-energy structures that have been previously reported for aluminum clusters. Here we only consider studies for which we were able to find the atomic coordinates of the discovered structures. ${ }^{28,29,58-60}$ All structures collected from the literature were reoptimized by DFT using the same settings as those used in GA_AL. For 25 of the 35 sizes, GA_AL found structures at least $1 \mathrm{meV} /$ atom lower in energy than the lowest-energy structure 
in the literature, and for another 7 sizes it identified the same lowest-energy structure as was available in the literature. For clusters of 22 atoms, GA_DFT found a structure that is structurally distinct from the lowest-energy structure reported in literature ${ }^{28}$ but has only slightly lower energy (by $0.15 \mathrm{meV} /$ atom). The GA_DFT algorithm discovered structures lower in energy than those discovered by GA_AL and the literature for 2 sizes. For clusters of 45 atoms, GA_DFT rediscovered the best-known structure from the literature but GA_AL did not. Detailed results are provided in Figure 5 and Table S4. A complete panel of lowest-energy clusters with 21 to 55 atoms is included in Figure S15 of the Supplementary Information and coordinates of these clusters are listed in a second Supplementary Information file.

Structures found using GA_AL have lower energies than the lowest-energy literature structures by an average of $16.81 \mathrm{meV} /$ atom, with a maximum of $51.81 \mathrm{meV} /$ atom at size $36(1.87 \mathrm{eV} / \mathrm{cluster})$. For the five sizes for which GA_AL did not discover the best structures (Figure 5a), the energies are no more than $4.0 \mathrm{meV} /$ atom above those of the structures with the lowest known energies. The preferred morphologies of the lowest-energy clusters of aluminum alter between layered closepacked structures with FCC stacking order and tetrahedrons. The cluster with 36 atoms is particularly stable relative to its neighboring sizes (see Section 7.2 of the Supplementary Information), suggesting that it may be particularly likely to be synthesizable. Experimentally, positively-charged $\mathrm{Al}$ clusters with around 36 atoms have been observed to have relatively high melting temperatures. ${ }^{63,64}$ 
a)

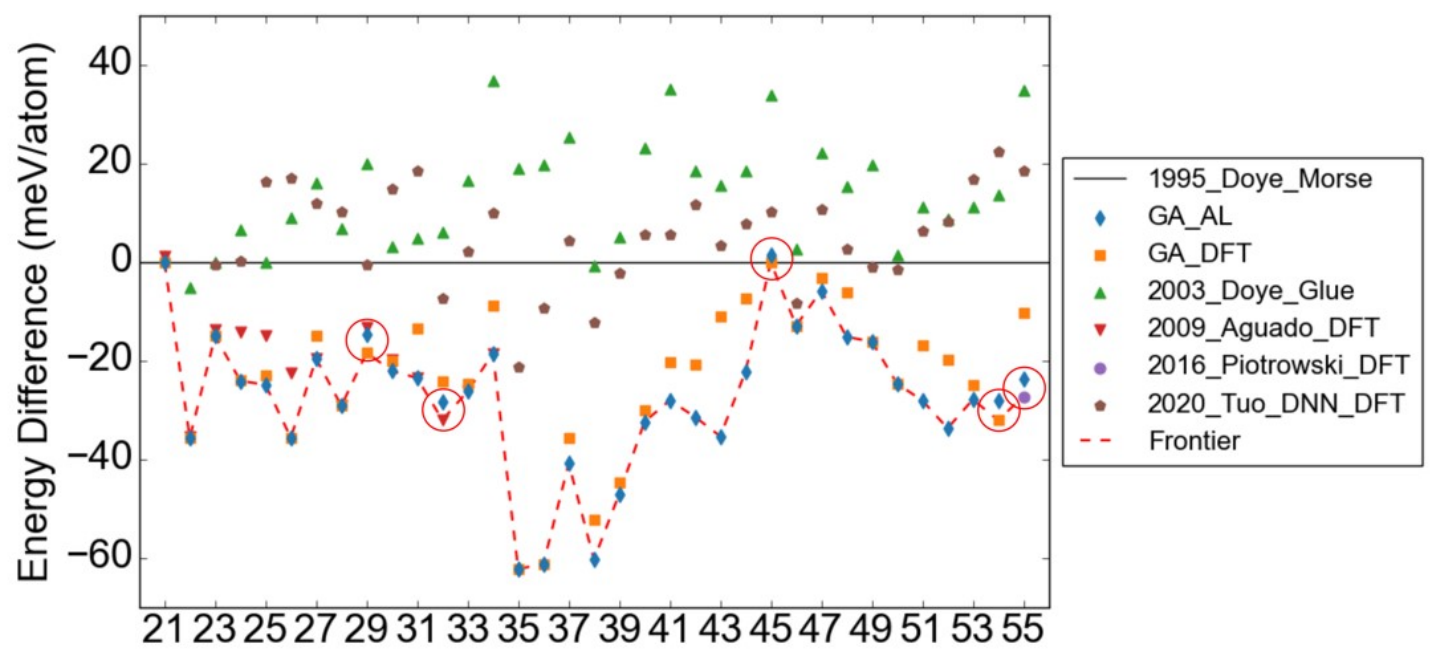

b)
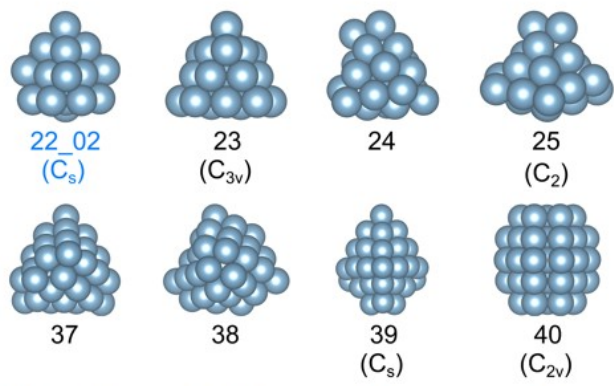

$\left(\mathrm{C}_{2}\right)$
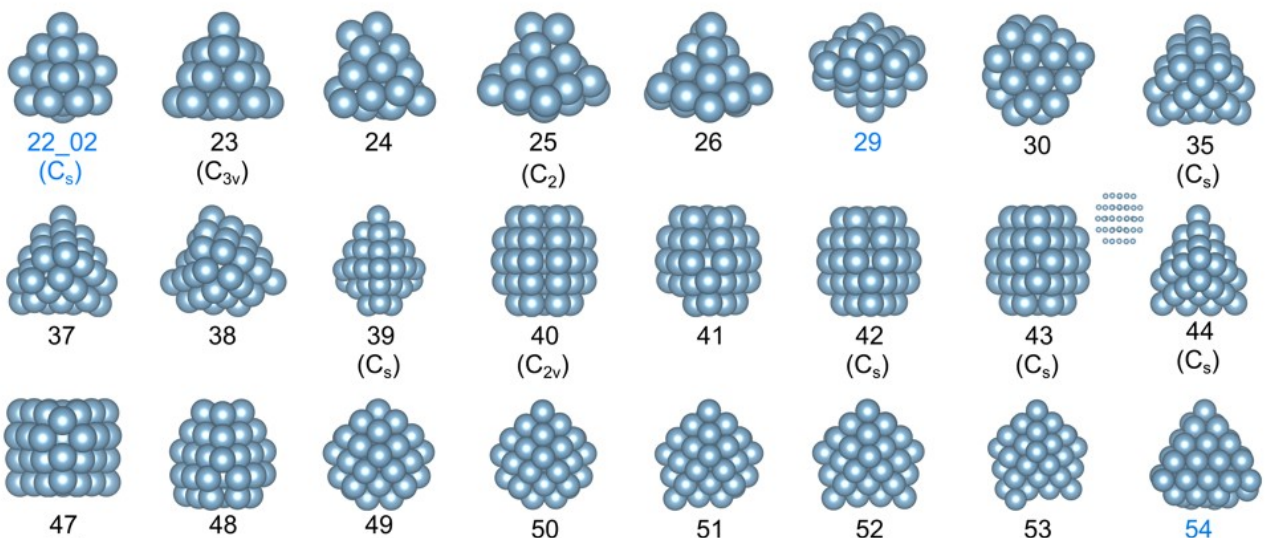

$\left(\mathrm{C}_{\mathrm{s}}\right)$
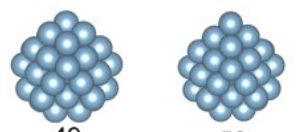

50
$\left(\mathrm{C}_{2}\right)$

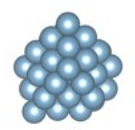

51

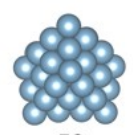

52
$\left(\mathrm{~S}_{4}\right)$

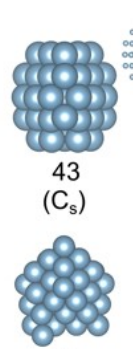

$\left(\mathrm{C}_{\mathrm{s}}\right)$

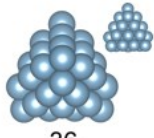

36

$\left(D_{2 d}\right)$

53

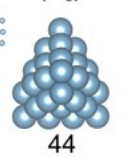

$\left(\mathrm{C}_{\mathrm{s}}\right)$

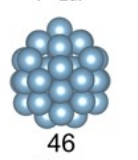

( $\left.\mathrm{C}_{\mathrm{s}}\right)$

Figure 5. Comparison between lowest-energy clusters reported in the literature and those discovered by GA_AL and GA_DFT. a) Energy differences of lowest-energy clusters from various methods relative to the energies of clusters reported by Doye and coworkers. ${ }^{58}$ The lowestenergy frontier is connected by a red dashed line, and the sizes for which GA_AL did not discover the best configurations are circled. "Morse", "Glue" and "DNN" in the legend represents the Morse potential, a glue potential and a deep neural network potential. b) New lowest-energy clusters discovered by GA_AL and GA_DFT. The labels of clusters identified by GA_DFT and not GA_AL are blue and corresponding symmetries are denoted in parentheses. Alternative views of representative layered close-packed structure (43) and tetrahedral clusters (36) are included above and to the right of the clusters.

\section{DISCUSSION}

Although the GA_AL approach presented here has clear advantages, including an order of magnitude acceleration compared to GA_DFT, there are potential areas for improvement. One is the relatively high energy prediction errors of MTP for nanoclusters compared with bulk systems. 
Benchmarks by Zuo and coworkers ${ }^{50}$ showed that MTP has energy errors generally less than 5 $\mathrm{meV} /$ atom and sometimes even lower than $1 \mathrm{meV} /$ atom for bulk elemental systems. However, for nanoclusters, as exhibited in Figure 3, validation energy errors are at the order of $10 \mathrm{meV} / \mathrm{atom}$ for small clusters with 24 atoms. They can be lowered by using smaller force weights, but the improvement comes at the expense of driving up force errors, which increases the possibility of creating artificial local minima. The relatively large energy errors increase the chance that the energy of the lowest-energy cluster is overestimated and never enters the pool. To mitigate this risk we used a relatively large pool size ( 25 clusters) to expand the energy window of pool clusters and raise the chance of the lowest-energy cluster being captured in the pool. A large pool has also been shown to increase the success rate of identifying the lowest-energy isomer due to the structural diversity of the pool, ${ }^{21}$ although at the expense of slowing convergence speed. ${ }^{20,21}$

We found that the energy window of pool clusters became narrower as the structure search continued, implying a high density of metastable states with energies close to the global minimum, especially for large clusters. This is not unexpected since the dimension of configuration space dramatically increases as system size grows. The relatively narrow energy window increases the chance of the pool missing the lowest-energy isomer, as the window size may be comparable to the error in MTP energy predictions. A possible workaround is to run GA_AL and then use the discovered low-energy clusters to seed a GA_DFT search. This would consume additional computational resources but decrease the uncertainty in the proposed lowest-energy structures.

Another area for improvement is the relationship between the extrapolation grades (used to identify structures that trigger retraining) and prediction errors. A high extrapolation grade normally implies an energy evaluation with high uncertainty, but a low grade does not necessarily guarantee an accurate prediction (see Figure S17 of Supplementary Information). In practice, we addressed this challenge by starting DFT re-optimization and retraining whenever the majority of clusters in the pool had MTP-calculated energies but not DFT-calculated energies. An alternative approach would be to implement similarity-based measurements of uncertainty, which might more accurately identify structures for which the prediction errors are likely to be large.

Although we have demonstrated that potentials trained on small clusters can be used to predict the structures of clusters about twice as large, it is not clear how well these potentials will work on significantly larger particles. If transferability can be retained up to larger clusters, the methods we have presented could be used to efficiently create a comprehensive datasets of cluster structures for small particles with structures that cannot be simply described as that of a truncated crystal.

\section{METHODS}

\section{Similarity measurement}

We quantify geometric similarity between two cluster structures of same size by a similarity score calculated using an approach based on the spectral decomposition of extended distance matrices. ${ }^{65}$ The score is non-negative and a smaller value implies higher similarity. Identical clusters have a score of 0 , and visually distinguishable clusters typically have a score above about 0.3 . The 
similarity measure is used to prevent geometrically similar clusters from being simultaneously included in the pool, which can improve the efficiency of $\mathrm{GA},{ }^{21}$ and to select diverse training data for the moment tensor potentials. ${ }^{55}$

\section{Genetic algorithm}

A genetic algorithm is a global optimization method inspired by the principles of natural selection. ${ }^{66}$ We developed our own code based off the pool-based Birmingham Parallel Genetic Algorithm ${ }^{23}$ with some variations. A pool of low-energy clusters of fixed size is maintained during the search. Initial clusters are generated by randomly distributing atoms in space. Once the pool is filled, genetic operations, namely, crossover and mutation, are applied to parent clusters selected from the pool to generate child clusters. Child clusters that are dissimilar to all pool clusters and have a lower energy than the pool cluster with the highest energy will replace the highest-energy pool cluster. Additional details of the genetic algorithm can be found in Section 1 of the Supplementary Information.

\section{Genetic algorithm with actively learned interatomic potentials}

To accelerate the genetic algorithm search for new stable nanoclusters, we use machine-learned interatomic potentials (to improve speed) trained on-the-fly using active learning (to maintain accuracy). We refer to this combination of genetic algorithms and active learning as "GA_AL". The active-learning query strategy uses the generalized D-Optimality criterion implemented in the MLIP package, ${ }^{46,47}$ which assigns unlabeled data an "extrapolation grade" based on a measure of the extent to which the unlabeled data is outside of the space spanned by the training data. An extrapolation grade above 1 implies extrapolation relative to the current training set and large errors should be expected, while a value below 1 indicates interpolation. ${ }^{47}$

The GA_AL runs batch retraining cycles and maintains a waitlist of structures to be included in the next cycle (Figure 6). Two extrapolation grade thresholds are used when determining whether a newly-generated cluster should be added to the waitlist. The first threshold, $\gamma_{\text {break }}$, is used to screen clusters before relaxation using MTP. The trained potential may struggle to relax clusters with extrapolation grades above this threshold, so they are automatically added to the waitlist. Structures with extrapolation grades below $\gamma_{\text {break }}$ are relaxed. If the extrapolation grade of the relaxed structure is greater than the second threshold, $\gamma_{\text {select }}$, then it too is added to the waitlist. In this work $\gamma_{\text {break }}$ was set to 10 for GA_AL initialized with untrained potential, as the default value recommend by MTP code. ${ }^{47} \mathrm{~A}$ looser value of 1000 was used for the pre-trained potential, as it is not as important to add training data to a potential that has already been trained. As-generated clusters with extrapolation grades about 1000 typically cannot be evaluated accurately by MTP potentials, but we found they could still be relaxed by MTP to reasonable configurations. Starting DFT relaxations from configurations pre-relaxed using MTP was used to reduce computational costs. The parameter $\gamma_{\text {select }}$ was set to 1.01 for all searches. When the waitlist reaches a user-defined value (here set to 5), the genetic algorithm is paused and a retraining cycle begins. All new clusters in the pool as well as clusters on the waitlist are relaxed using DFT and added to the training set. Before retraining the potential, a similarity screen is applied to select the most geometrically 
diverse set of configurations from all relaxation steps (discussed below), which maximizes structural diversity and reduces training cost.

Because of the uncertainty in MTP-predicted energies, there is a risk that the pool over time becomes polluted with structures with erroneously low MTP-predicted energies. To mitigate this risk, a retraining cycle is also started whenever a majority of the clusters in the pool $(>50 \%)$ have energies that were calculated using MTP and not DFT. GA_AL is considered to be converged when no cluster with an energy lower than the lowest-energy pool cluster has been found for 4000 new clusters.

When initializing GA_AL with pre-trained potentials, it is beneficial to switch off retraining at the beginning of the search. In this approach, extrapolating clusters are discarded and new ones are regenerated until they are interpolating, allowing the GA to more fully explore the potential energy surface of the pre-trained potential. We did this for the first 5000 clusters in GA_AL runs initialized with mixed-size potentials when generating clusters with 41 to 55 atoms. Additional discussion and justification for this approach are provided in Section 1.4 of the Supplementary Information.

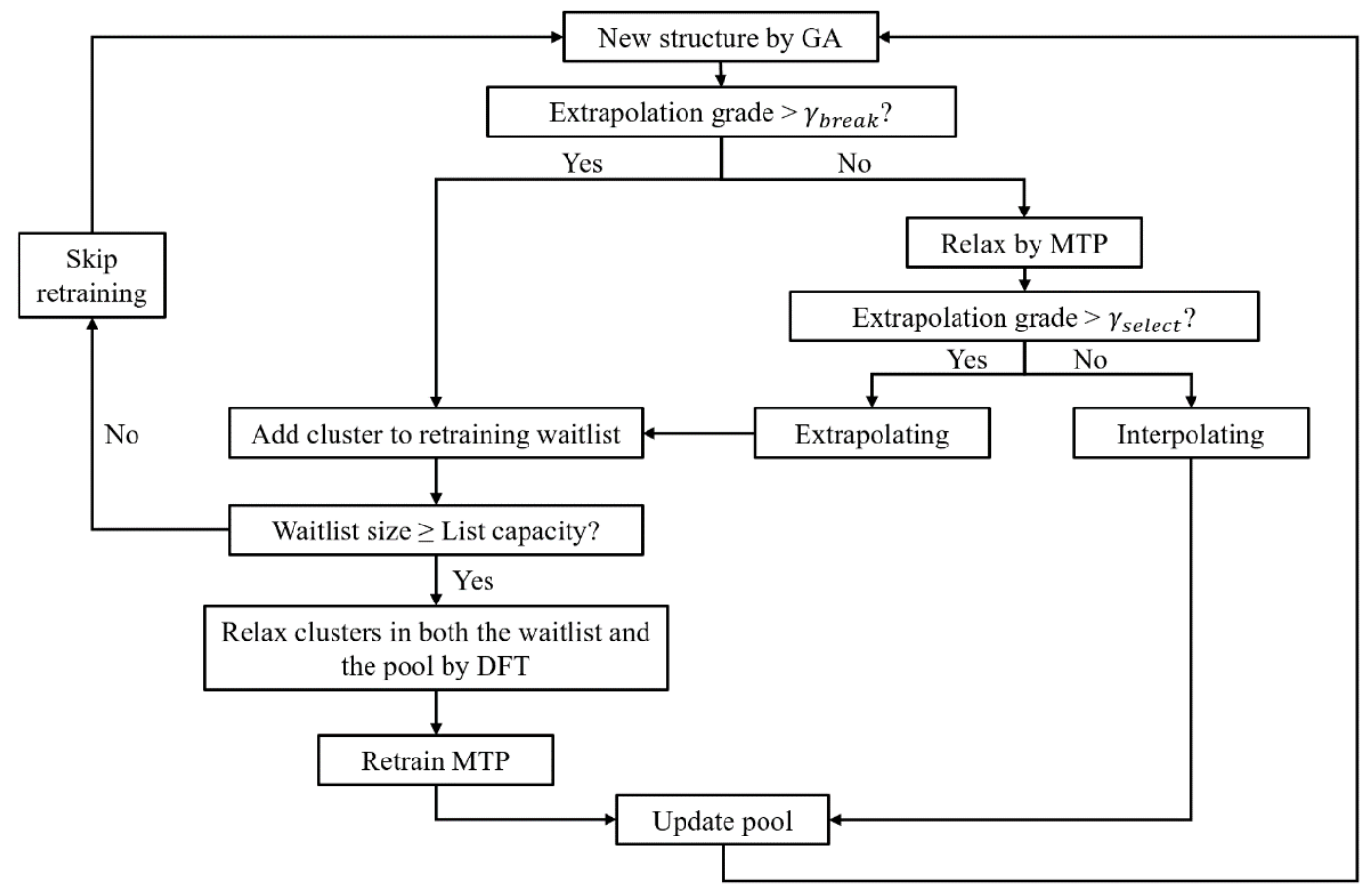

Figure 6. Schematic workflow of genetic algorithm with on-the-fly active learning (GA_AL). A double-threshold scheme is employed in which a looser threshold, $\gamma_{\text {break }}$, and a tighter threshold, $\gamma_{\text {select }}$, are used to determine extrapolation of as-generated structures and MTP-relaxed structures, respectively. Retraining starts when the waitlist exceeds a user-defined capacity, which was set to 5 in this work. 


\section{Moment tensor potentials}

We used the MLIP package ${ }^{47}$ to train moment tensor potentials. The hyperparameters for training include potential complexity, energy weight, force weight and stress weight. Potential complexity is characterized by the maximum level of moments, $l e v_{\max }$, of basis functions. ${ }^{45,47}$ The energy weight was always set to 1 , so the force weight can be seen as the weight of force components relative to the weight of the energy. The stress weight was set to 0 since it is irrelevant in the case of clusters due to the lack of lattice. We generated potentials with $l e v_{\max }=14$ and a force weight of $1 / 1000$ relative to the energy weight, to balance between accuracy and training cost, as shown in the Results section and Section 2.2 of Supplementary Information. The inner and outer cutoff radii defining the local atomic neighborhood were set to the default values of $2 \AA$ and $5 \AA$, and eight radial basis functions were used. ${ }^{47}$ A maximum number of 5000 training iterations were allowed for potential fitting. This limit was never reached, as the maximum number of training iterations in any GA_AL run was 1399.

\section{Training data selection for pre-trained potentials}

To select the training data for the pre-trained mixed-size potentials, we used a diversity-based strategy and an energy-based strategy to select structurally diverse structures from DFT relaxations and to improve accuracy in the low-lying regions of the potential energy surface. All DFT calculations were collected from GA_DFT and GA_AL runs on clusters with 21-40 atoms. For each of the constituent cluster sizes, relaxation trajectories were only kept if the corresponding local ground states have similarity scores larger than 0.3 with the local ground states of all other trajectories already included in the training set. Within each trajectory, only dissimilar ionic steps were selected as well. We accomplished this by including the fully relaxed structure and iterating backwards through the relaxation until encountering a structure with a similarity score, relative to the most recently-added structure, that was at least 0.3 . That structure was then added to the training set, and we repeated this procedure until all relaxation steps were exhausted. This diversity-based strategy was applied throughout this work, in data preparation processes for both training and validation sets.

Following the similarity screening, we performed an energy-based selection strategy. First, all structures that passed through the diversity screening were grouped into sets based on the number of atoms in the cluster. $50 \%$ of the training data selected from each set consisted of the structures with lowest energy, $10 \%$ consisted of the structures with the highest energy, and the remaining $40 \%$ were randomly from the remaining ionic steps.

A total of 3000 structures were selected for each of the training sets. For potentials trained with clusters of multiple sizes (the ones labelled by "odd", "even" and "all" in Figure 3), equal numbers of structures were chosen for each constituent sizes. For potentials trained with clusters of a single size, all 3000 structures were chosen from clusters of that size. The training set for the potential trained with clusters of 21 atoms only contains 2136 structures after the diversity filtering of DFT calculations, and were all included in the training set. 


\section{Validation data selection for pre-trained potentials}

We collected validation data for mixed-size potentials from GA_DFT runs on clusters with 50-55 atoms. The diversity-based strategy discussed above was used to select a structurally diverse set of structures. The validation sets contain around 3000 structures for each size (details are provided in Section 3.1 of the Supplementary Information).

\section{Training data selection for on-the-fly retraining in GA_AL}

We also use similarity filtering, as described above, to select structures for retraining on-the-fly. Similarity filtering is used to select distinct clusters from each relaxation trajectory during active learning. We used a tight similarity threshold of 0.3 on small to medium clusters $(21-40)$ and a looser threshold of 0.15 on large clusters (41-55). The looser threshold is meant to increase the fraction of available data used for retraining for large clusters, as generating this data is computationally more expensive. We do not check similarity between new training data and all existing data on-the-fly, as this is computationally costly.

\section{Comparison of GA_AL against GA_DFT}

Both GA_AL and GA_DFT were run using the same set of genetic algorithm parameters (see Section 1.3 of the Supplementary Information) on all 24 cores of Intel E5-2680 V3 processors. The GA_AL runs were performed until 4000 consecutive new clusters had been generated without identifying a new lowest-energy cluster. GA_DFT runs were performed for at least the same amount of time as GA_AL runs for fair comparison. For large clusters with 50-55 atoms, GA_DFT searches were executed for a much longer time of 21 days, to reach comparable energy levels as GA_AL runs (see also Section 1.2 of the Supplementary Information). The reported time spent for GA_AL includes the time spent in the genetic algorithm search, time required to generate training data using DFT, and time spent training the interatomic potential. For GA_AL runs initialized with pre-trained potentials, the time spent pre-training the potential was also included. The only difference between the GA_AL and GA_DFT algorithms was that GA_AL used moment tensor potentials with active learning, whereas GA_DFT used only DFT for relaxation. Low-energy structures identified in GA_AL were re-optimized using DFT at the retraining stage and only DFTevaluated energies were reported at the end, to ensure an ab initio level of accuracy.

To determine whether a new low-energy structure had been found, we considered both the DFTcalculated energy and similarity scores. A cluster that is lower in energy by at least $1 \mathrm{meV} / \mathrm{atom}$ and at the same time has a similarity score, compared to the existing lowest-energy structure, that is greater than 0.3 is identified as a new lowest-energy cluster. Clusters that have a total energy within $1 \mathrm{meV} /$ atom to the existing lowest-energy cluster but are structurally dissimilar are considered as energetically similar clusters and are not counted as new lowest-energy clusters. Borderline cases were inspected manually. More details of how we determined whether the algorithm had found a new lowest-energy structure can be found in Section 6 of the Supplementary Information. 


\section{DFT calculations}

All DFT calculations were carried out using the Vienna ab-initio simulation package (VASP) ${ }^{62,67-}$ ${ }^{69}$ with the Perdew-Burke-Ernzerhof (PBE) exchange-correlation functional. ${ }^{70-72}$ The projector augmented wave (PAW) dataset shipped with VASP with the title "PAW_PBE Al 04Jan2001" was used. ${ }^{73,74}$ Reciprocal space was sampled by a single $k$-point at the $\Gamma$ point and the kinetic energy cutoff for the plane-wave basis was set to $240 \mathrm{eV}$. The electronic self-consistency loop was considered to reach convergence when subsequent steps had an energy difference below $10^{-5} \mathrm{eV}$ and the convergence criterion for ionic relaxation was set to a force difference below $0.01 \mathrm{eV} / \AA$. Our dataset ${ }^{75}$ shows that ground state Al nanoclusters with even sizes above 18 and odd sizes above 7 have net spins of $0 \mu_{B}$ and $1 \mu_{B}$, respectively (see Section 4 of Supplementary Information). Therefore, all ab initio calculations fix the magnetic moment to $0 \mu_{B}$ for even-sized clusters and to $1 \mu_{B}$ for odd-sized clusters using the parameter NUPDOWN in VASP.

\section{DATA AVAILABILITY}

Structure files for the lowest-energy clusters with 21 to 55 atoms are listed in a separate Supplementary Information file in the XYZ format.

\section{CODE AVAILABILITY}

Our implementation of the GA_AL procedure is open-sourced under the Apache License 2.0 at https://gitlab.com/muellergroup/cluster-ga. The code also supports genetic algorithm searches using only DFT or interatomic potentials through interfaces with VASP and LAMMPS. Input templates and documentation of input parameters can be found in the repository.

\section{ACKONWLEDGEMENT}

We thank Prof. Alexander V. Shapeev for providing helpful guidance in using the MLIP package. The work was supported by the Office of Naval Research under the grant No. ONR MURI N00014-15-1-2681. Calculations were performed using computational resources from the Maryland Advanced Research Computing Cluster (MARCC), the Stampede2 supercomputer at the Texas Advanced Computer Center (TACC) and the Gordon supercomputer in Department of Defense High Performance Computing Modernization Program. TACC resources were provided through the XSEDE program with NSF award DMR-140068. Images of the atomic structures of clusters were generated using VESTA. ${ }^{76}$

\section{AUTHOR CONTRIBUTIONS}

T.M. conceptualized and managed the project. Y.W., S.L., P.L., S.N., A.H. and T.M. developed the software. Y.W., S.L. and T.M. wrote the manuscript. Y.W. performed the benchmarks on MTP. Y.W., S.L., and S.M. carried out GA searches and analyzed data. S.L., Y.W. and S.M. collected structures from literatures. All the authors proposed, discussed, or developed ideas that improved the performance of the GA_AL procedure and the quality of the data. 


\section{References}

1 Kang, X., Li, Y., Zhu, M. \& Jin, R. Atomically precise alloy nanoclusters: syntheses, structures, and properties. Chemical Society Reviews 49, 6443-6514, doi:10.1039/C9CS00633H (2020).

2 Jena, P. \& Castleman, A. W. Clusters: A bridge across the disciplines of physics and chemistry. Proceedings of the National Academy of Sciences 103, 10560, doi:10.1073/pnas.0601782103 (2006).

3 Gawande, M. B. et al. $\mathrm{Cu}$ and $\mathrm{Cu}$-Based Nanoparticles: Synthesis and Applications in Catalysis. Chemical Reviews 116, 3722-3811, doi:10.1021/acs.chemrev.5b00482 (2016).

4 Liu, L. \& Corma, A. Metal Catalysts for Heterogeneous Catalysis: From Single Atoms to Nanoclusters and Nanoparticles. Chemical Reviews 118, 4981-5079, doi:10.1021/acs.chemrev.7b00776 (2018).

5 Jin, R., Zeng, C., Zhou, M. \& Chen, Y. Atomically Precise Colloidal Metal Nanoclusters and Nanoparticles: Fundamentals and Opportunities. Chemical Reviews 116, 10346-10413, doi:10.1021/acs.chemrev.5b00703 (2016).

6 Li, G. \& Jin, R. Atomically Precise Gold Nanoclusters as New Model Catalysts. Accounts of Chemical Research 46, 1749-1758, doi:10.1021/ar300213z (2013).

7 Saha, K., Agasti, S. S., Kim, C., Li, X. \& Rotello, V. M. Gold Nanoparticles in Chemical and Biological Sensing. Chemical Reviews 112, 2739-2779, doi:10.1021/cr2001178 (2012).

8 Kang, X. \& Zhu, M. Tailoring the photoluminescence of atomically precise nanoclusters. Chemical Society Reviews 48, 2422-2457, doi:10.1039/C8CS00800K (2019).

9 Jin, R. Atomically precise metal nanoclusters: stable sizes and optical properties. Nanoscale 7, 1549-1565, doi:10.1039/C4NR05794E (2015).

10 White, R. J., Luque, R., Budarin, V. L., Clark, J. H. \& Macquarrie, D. J. Supported metal nanoparticles on porous materials. Methods and applications. Chemical Society Reviews 38, 481-494, doi:10.1039/B802654H (2009).

11 Martin, T. P. Shells of atoms. Physics Reports 273, 199-241, doi:https://doi.org/10.1016/0370-1573(95)00083-6 (1996).

12 Ferrando, R., Jellinek, J. \& Johnston, R. L. Nanoalloys: From Theory to Applications of Alloy Clusters and Nanoparticles. Chemical Reviews 108, 845-910, doi:10.1021/cr040090g (2008).

13 Wales, D. J. \& Doye, J. P. K. Global Optimization by Basin-Hopping and the Lowest Energy Structures of Lennard-Jones Clusters Containing up to 110 Atoms. The Journal of Physical Chemistry A 101, 5111-5116, doi:10.1021/jp970984n (1997).

14 Wales, D. J. \& Scheraga, H. A. Global Optimization of Clusters, Crystals, and Biomolecules. Science 285, 1368, doi:10.1126/science.285.5432.1368 (1999).

15 Pickard, C. J. \& Needs, R. J. Ab initio random structure searching. J Phys-Condens Mat 23 (2011).

16 Call, S. T., Zubarev, D. Y. \& Boldyrev, A. I. Global minimum structure searches via particle swarm optimization. Journal of Computational Chemistry 28, 1177-1186, doi:https://doi.org/10.1002/jcc.20621 (2007).

17 Wang, Y., Lv, J., Zhu, L. \& Ma, Y. Crystal structure prediction via particle-swarm optimization. Physical Review B 82, 094116, doi:10.1103/PhysRevB.82.094116 (2010).

18 Weigend, F. \& Ahlrichs, R. Quantum chemical treatments of metal clusters. Philosophical Transactions of the Royal Society a-Mathematical Physical and Engineering Sciences 368, 1245-1263, doi:10.1098/rsta.2009.0268 (2010). 
19 Ahlrichs, R. \& D. Elliott, S. Clusters of aluminium, a density functional study. Phys Chem Chem Phys 1, 13-21, doi:10.1039/A807713D (1999).

20 Johnston, R. L. Evolving better nanoparticles: Genetic algorithms for optimising cluster geometries. Dalton Transactions, 4193-4207, doi:10.1039/B305686D (2003).

21 Vilhelmsen, L. B. \& Hammer, B. A genetic algorithm for first principles global structure optimization of supported nano structures. J Chem Phys 141 (2014).

22 Tipton, W. W. \& Hennig, R. G. A grand canonical genetic algorithm for the prediction of multi-component phase diagrams and testing of empirical potentials. Journal of Physics: Condensed Matter 25, 495401, doi:10.1088/0953-8984/25/49/495401 (2013).

23 Shayeghi, A., Götz, D., Davis, J. B. A., Schäfer, R. \& Johnston, R. L. Pool-BCGA: a parallelised generation-free genetic algorithm for the ab initio global optimisation of nanoalloy clusters. Phys Chem Chem Phys 17, 2104-2112, doi:10.1039/C4CP04323E (2015).

24 Deaven, D. M. \& Ho, K. M. Molecular-Geometry Optimization with a Genetic Algorithm. Phys Rev Lett 75, 288-291 (1995).

25 Vargas, J. A., Buendía, F. \& Beltrán, M. R. New Au $(\mathrm{N}=27-30)$ Lowest Energy Clusters Obtained by Means of an Improved DFT-Genetic Algorithm Methodology. The Journal of Physical Chemistry C 121, 10982-10991, doi:10.1021/acs.jpcc.6b12848 (2017).

26 Davis, J. B. A., Shayeghi, A., Horswell, S. L. \& Johnston, R. L. The Birmingham parallel genetic algorithm and its application to the direct DFT global optimisation of $\operatorname{Ir}_{\mathrm{N}}(\mathrm{N}=10$ 20) clusters. Nanoscale 7, 14032-14038, doi:10.1039/C5NR03774C (2015).

27 Drebov, N. \& Ahlrichs, R. Structures of $\mathrm{Al}_{\mathrm{n}}$, its anions and cations up to $\mathrm{n}=34$ : A theoretical investigation. The Journal of Chemical Physics 132, 164703, doi:10.1063/1.3403692 (2010).

28 Aguado, A. \& López, J. M. Structures and stabilities of $\mathrm{Al}_{n}{ }^{+}, \mathrm{Al}_{\mathrm{n}}$, and $\mathrm{Al}_{\mathrm{n}}{ }^{-}(\mathrm{n}=13-34)$ clusters. The Journal of Chemical Physics 130, 064704, doi:10.1063/1.3075834 (2009).

29 Doye, J. P. K. A model metal potential exhibiting polytetrahedral clusters. The Journal of Chemical Physics 119, 1136-1147, doi:10.1063/1.1574797 (2003).

30 Xiang, Y., Jiang, H., Cai, W. \& Shao, X. An Efficient Method Based on Lattice Construction and the Genetic Algorithm for Optimization of Large Lennard-Jones Clusters. The Journal of Physical Chemistry A 108, 3586-3592, doi:10.1021/jp037780t (2004).

31 Galvão, B. R. L. \& Viegas, L. P. What Electronic Structure Method Can Be Used in the Global Optimization of Nanoclusters? The Journal of Physical Chemistry A 123, 1045410462, doi:10.1021/acs.jpca.9b09309 (2019).

32 Behler, J. Perspective: Machine learning potentials for atomistic simulations. The Journal of Chemical Physics 145, 170901, doi:10.1063/1.4966192 (2016).

33 Mueller, T., Hernandez, A. \& Wang, C. Machine learning for interatomic potential models. The Journal of Chemical Physics 152, 050902, doi:10.1063/1.5126336 (2020).

34 Schmidt, J., Marques, M. R. G., Botti, S. \& Marques, M. A. L. Recent advances and applications of machine learning in solid-state materials science. Npj Comput Mater 5, 83, doi:10.1038/s41524-019-0221-0 (2019).

35 Deringer, V. L., Caro, M. A. \& Csányi, G. Machine Learning Interatomic Potentials as Emerging Tools for Materials Science. Advanced Materials 31, 1902765, doi:10.1002/adma.201902765 (2019). 
36 Ramprasad, R., Batra, R., Pilania, G., Mannodi-Kanakkithodi, A. \& Kim, C. Machine learning in materials informatics: recent applications and prospects. Npj Comput Mater 3, 54, doi:10.1038/s41524-017-0056-5 (2017).

37 Quaranta, V., Behler, J. \& Hellström, M. Structure and Dynamics of the LiquidWater/Zinc-Oxide Interface from Machine Learning Potential Simulations. The Journal of Physical Chemistry C 123, 1293-1304, doi:10.1021/acs.jpcc.8b10781 (2019).

38 Houchins, G. \& Viswanathan, V. An accurate machine-learning calculator for optimization of Li-ion battery cathodes. 153, 054124, doi:10.1063/5.0015872 (2020).

39 Chiriki, S. \& Bulusu, S. S. Modeling of DFT quality neural network potential for sodium clusters: Application to melting of sodium clusters (Na20 to Na40). Chemical Physics Letters 652, 130-135, doi:https://doi.org/10.1016/j.cplett.2016.04.013 (2016).

40 Bartók, A. P., Payne, M. C., Kondor, R. \& Csányi, G. Gaussian Approximation Potentials: The Accuracy of Quantum Mechanics, without the Electrons. Phys Rev Lett 104, 136403, doi:10.1103/PhysRevLett.104.136403 (2010).

41 Bartók, A. P., Kondor, R. \& Csányi, G. On representing chemical environments. Physical Review B 87, 184115, doi:10.1103/PhysRevB.87.184115 (2013).

42 Szlachta, W. J., Bartók, A. P. \& Csányi, G. Accuracy and transferability of Gaussian approximation potential models for tungsten. Physical Review B 90, 104108, doi:10.1103/PhysRevB.90.104108 (2014).

43 Thompson, A. P., Swiler, L. P., Trott, C. R., Foiles, S. M. \& Tucker, G. J. Spectral neighbor analysis method for automated generation of quantum-accurate interatomic potentials. $J$ Comput Phys 285, 316-330, doi:https://doi.org/10.1016/j.jcp.2014.12.018 (2015).

44 Wood, M. A. \& Thompson, A. P. Extending the accuracy of the SNAP interatomic potential form. The Journal of Chemical Physics 148, 241721, doi:10.1063/1.5017641 (2018).

45 Shapeev, A. V. Moment Tensor Potentials: A Class of Systematically Improvable Interatomic Potentials. Multiscale Model Sim 14, 1153-1173 (2016).

46 Podryabinkin, E. V.\& Shapeev, A. V. Active learning of linearly parametrized interatomic potentials. Comp Mater Sci 140, 171-180, doi:10.1016/j.commatsci.2017.08.031 (2017).

47 Novikov, I. S., Gubaev, K., Podryabinkin, E. V. \& Shapeev, A. V. The MLIP package: moment tensor potentials with MPI and active learning. Machine Learning: Science and Technology 2, 025002, doi:10.1088/2632-2153/abc9fe (2021).

48 Drautz, R. Atomic cluster expansion for accurate and transferable interatomic potentials. Physical Review B 99, 014104, doi:10.1103/PhysRevB.99.014104 (2019).

49 Hernandez, A., Balasubramanian, A., Yuan, F., Mason, S. A. M. \& Mueller, T. Fast, accurate, and transferable many-body interatomic potentials by symbolic regression. $\mathrm{Npj}$ Comput Mater 5, 112, doi:10.1038/s41524-019-0249-1 (2019).

50 Zuo, Y. X. et al. Performance and Cost Assessment of Machine Learning Interatomic Potentials. J Phys Chem A 124, 731-745, doi:10.1021/acs.jpca.9b08723 (2020).

51 Hartke, B. Global geometry optimization of clusters guided by $\mathrm{N}$-dependent model potentials. Chemical Physics Letters 258, 144-148, doi:https://doi.org/10.1016/00092614(96)00629-X (1996).

52 Deringer, V. L., Proserpio, D. M., Csányi, G. \& Pickard, C. J. Data-driven learning and prediction of inorganic crystal structures. Faraday Discussions 211, 45-59, doi:10.1039/C8FD00034D (2018). 
53 Podryabinkin, E. V., Tikhonov, E. V., Shapeev, A. V. \& Oganov, A. R. Accelerating crystal structure prediction by machine-learning interatomic potentials with active learning. Physical Review B 99, 064114, doi:10.1103/PhysRevB.99.064114 (2019).

54 Gubaev, K., Podryabinkin, E. V., Hart, G. L. W. \& Shapeev, A. V. Accelerating highthroughput searches for new alloys with active learning of interatomic potentials. Comp Mater Sci 156, 148-156 (2019).

55 Bernstein, N., Csányi, G. \& Deringer, V. L. De novo exploration and self-guided learning of potential-energy surfaces. Npj Comput Mater 5, 99, doi:10.1038/s41524-019-0236-6 (2019).

56 Kolsbjerg, E. L., Peterson, A. A. \& Hammer, B. Neural-network-enhanced evolutionary algorithm applied to supported metal nanoparticles. Physical Review B 97, 195424, doi:10.1103/PhysRevB.97.195424 (2018).

57 Tong, Q., Xue, L., Lv, J., Wang, Y. \& Ma, Y. Accelerating CALYPSO structure prediction by data-driven learning of a potential energy surface. Faraday Discussions 211, 31-43, doi:10.1039/C8FD00055G (2018).

58 Doye, J. P. K., Wales, D. J. \& Berry, R. S. The effect of the range of the potential on the structures of clusters. The Journal of Chemical Physics 103, 4234-4249, doi:10.1063/1.470729 (1995).

59 Piotrowski, M. J. et al. Theoretical Study of the Structural, Energetic, and Electronic Properties of 55-Atom Metal Nanoclusters: A DFT Investigation within van der Waals Corrections, Spin-Orbit Coupling, and PBE+U of 42 Metal Systems. The Journal of Physical Chemistry C 120, 28844-28856, doi:10.1021/acs.jpcc.6b10404 (2016).

60 Tuo, P., Ye, X. B. \& Pan, B. C. A machine learning based deep potential for seeking the low-lying candidates of Al clusters. The Journal of Chemical Physics 152, 114105, doi:10.1063/5.0001491 (2020).

61 Nyshadham, C. et al. Machine-learned multi-system surrogate models for materials prediction. Npj Comput Mater 5, 51, doi:10.1038/s41524-019-0189-9 (2019).

62 Kresse, G. \& Furthmuller, J. Efficient iterative schemes for ab initio total-energy calculations using a plane-wave basis set. Physical Review B 54, 11169-11186 (1996).

63 Neal, C. M., Starace, A. K. \& Jarrold, M. F. Melting transitions in aluminum clusters: The role of partially melted intermediates. Physical Review B 76, 054113, doi:10.1103/PhysRevB.76.054113 (2007).

64 Aguado, A. \& Jarrold, M. F. Melting and Freezing of Metal Clusters. Annual Review of Physical Chemistry 62, 151-172, doi:10.1146/annurev-physchem-032210-103454 (2011).

65 Li, X.-T., Yang, X.-B. \& Zhao, Y.-J. Geometrical eigen-subspace framework based molecular conformation representation for efficient structure recognition and comparison. The Journal of Chemical Physics 146, 154108, doi:10.1063/1.4981212 (2017).

66 Holland, J. H. Adaptation in natural and artificial systems. (University of Michigan Press, 1975).

67 Kresse, G. \& Hafner, J. Ab initio molecular dynamics for liquid metals. Physical Review $B$ 47, 558-561, doi:10.1103/PhysRevB.47.558 (1993).

68 Kresse, G. \& Hafner, J. Ab initio molecular-dynamics simulation of the liquid-metal-amorphous-semiconductor transition in germanium. Physical Review B 49, 14251-14269, doi:10.1103/PhysRevB.49.14251 (1994).

69 Kresse, G. \& Furthmuller, J. Efficiency of ab-initio total energy calculations for metals and semiconductors using a plane-wave basis set. Comp Mater Sci 6, 15-50 (1996). 
70 Perdew, J. P., Burke, K. \& Wang, Y. Generalized gradient approximation for the exchangecorrelation hole of a many-electron system. Physical Review B 54, 16533-16539, doi:10.1103/PhysRevB.54.16533 (1996).

71 Perdew, J. P., Burke, K. \& Ernzerhof, M. Generalized Gradient Approximation Made Simple. Phys Rev Lett 77, 3865-3868, doi:10.1103/PhysRevLett.77.3865 (1996).

72 Perdew, J. P. \& Yue, W. Accurate and simple density functional for the electronic exchange energy: Generalized gradient approximation. Physical Review B 33, 8800-8802, doi:10.1103/PhysRevB.33.8800 (1986).

73 Blöchl, P. E. Projector augmented-wave method. Physical Review B 50, 17953-17979, doi:10.1103/PhysRevB.50.17953 (1994).

74 Kresse, G. \& Joubert, D. From ultrasoft pseudopotentials to the projector augmented-wave method. Physical Review B 59, 1758-1775, doi:10.1103/PhysRevB.59.1758 (1999).

75 Manna, S. et al. A Database to Enable the Design and Discovery of Atomically Precise Nanoclusters. (Manuscript in preparation).

76 Momma, K. \& Izumi, F. VESTA 3 for three-dimensional visualization of crystal, volumetric and morphology data. Journal of Applied Crystallography 44, 1272-1276, doi:10.1107/s0021889811038970 (2011). 


\section{Supplementary Information}

\section{for \\ "Accelerated Prediction of Atomically Precise Cluster Structures Using On-the-fly Machine Learning"}

Yunzhe Wang, Shanping Liu, Peter Lile, Sam Norwood, Alberto Hernandez, Sukriti Manna, and Tim Mueller*

Department of Materials Science and Engineering, Johns Hopkins University, Baltimore, MD 21218

*Corresponding Author Email: tmueller@jhu.edu

\section{Genetic Algorithm}

A genetic algorithm (GA) is a global optimization method inspired by the principle of natural selection. ${ }^{1}$ Genetic algorithms have been successfully applied to discover ground state configurations for a diverse set of systems including elemental metal clusters, ${ }^{2-5}$ binary and ternary alloys,${ }^{6-8}$ crystals,,${ }^{9,10}$ oxides, ${ }^{11}$ perovskites, ${ }^{12}$ and metal organic frameworks (MOFs). ${ }^{13}$ The efficiency of genetic algorithms comes from the fact that they can identify low-energy local atomic motifs and re-use them when generating candidate structures. ${ }^{3}$ This section provides additional details about the genetic algorithm (GA) implementation used in this work.

\subsection{Workflow of GA_DFT}

Our core implementation of the genetic algorithm, which we refer to as GA_DFT, uses only density functional theory (DFT) to evaluate energies. Our approach is based off the pool-based Birmingham Parallel Genetic Algorithm (BPGA) ${ }^{5}$ with some variations. In a pool-based GA, a fixed-size pool is maintained and comprises the lowest energy clusters among all structures that have been evaluated. This ensures that after each update the pool is at least as good as the old one. In this section we describe the general workflow of our code, with the specific parameters used in this work reported in later sections.

The GA has two phases: an initial phase when the pool starts off unfilled, and the genetic phase when the pool is full and child structure are generated by genetic operations. During the initial phase, structures can be generated by either a random distribution of atoms or by a seeding operation. We used the former method of random distribution of atoms in this work. With the former method, a cluster is generated by randomly distributing atoms in a cubic box with a side length of $2 r \sqrt[3]{N}$ where $r$ is the atomic radius of corresponding element and $N$ is the number of atoms. This approach ensures cluster volumes grow proportionately with the system size yet are not too sparsely populated.

In the seeding operation, new structures are generated from seed structures which are typically known low-energy structures with different numbers of atoms. Atoms are either randomly added or subtracted from the seed structure until it reaches the target size. If a seed cluster consists of a 
different element, the atomic coordinates and cell size are scaled proportionately to the ratio of atomic radii. Although it is available in the open-source code, this operation was not used in this work.

Once the pool has been filled, the genetic algorithm begins to generate clusters using one of the three genetic operations: seeding (as described above), mutation, and crossover.

In a mutation operation, a parent cluster is randomly selected from the pool and a randomly chosen subset of atoms is either globally displaced or rotated around the center of mass to create a child configuration.

In a crossover operation, a roulette wheel scheme is used to pick pairs of parents from the pool, and the probability of a cluster being chosen is proportional to its selectability, which is designed to reward low-energy clusters and panelize those that have been picked many times. ${ }^{3,4}$ The selectability of $i^{\text {th }}$ cluster is defined as

$$
S_{i}=f_{i} \times v_{i}
$$

where $f_{i}$ represents the fitness of the $i^{\text {th }}$ cluster and $v_{i}$ is a penalty term that depends on parenting frequency. The fitness is defined using a hyperbolic function of a normalized total energy,

$$
f_{i}=1 / 2\left[1-\tanh \left(2 \rho_{i}-1\right)\right]
$$

where the normalized energy $\rho_{i}$ is given by

$$
\rho_{i}=\frac{E_{i}-E_{\min }}{E_{\max }-E_{\min }},
$$

where $E_{\min }$ and $E_{\max }$ are the lowest and highest energies of the clusters in the pool. ${ }^{3}$ The lower total energy one cluster has relative to the others, the higher probability it has to be selected as parent. The penalty term is formulated to avoid overusing of the same cluster and is calculated as

$$
v_{i}=\frac{1}{1+\sqrt{n_{i}}},
$$

where $n_{i}$ stands for the number of times the $i^{\text {th }}$ cluster has been picked as one of the parents in crossover during the entire GA run. It has been shown that the use of both the fitness function and the frequency penalty to determine selectability can accelerate the convergence speed and increase the success rate of GA. ${ }^{3,4}$ Once a pair of parents is chosen, a child cluster is created by the cut-andsplice operation devised by Deaven and Ho. ${ }^{14}$ In addition to the common practice in which a child cluster inherits equal share of total atoms from each parent (even), varying percentages are allowed. The inheritance ratio can be proportional to fitness of clusters (weighted) or be randomly decided (random).

Newly generated configurations from all methods are checked for occurrences of atom overlap. Two atoms are considered as overlapping if their pairwise distances are smaller than $80 \%$ of the 
sum of atomic radii. If overlap exists, the corresponding cluster is regenerated by the same type of operation until it clears the examination.

Following successful cluster generation, the clusters are put in simulation boxes with lengths that allow at least $10 \AA$ between adjacent periodic images, and the atomic coordinates are relaxed using either density functional theory (DFT) or interatomic potentials. Currently, our code supports VASP $^{15-17}$ and LAMMPS, ${ }^{18}$ but any other energy-evaluation methods can be applied. Once child clusters are relaxed, they are compared with pool clusters. Child clusters which have lower energies than pool clusters and are structurally unique (defined here as having a similarity score above 0.3 ) are added to the pool, and the highest energy pool clusters are removed to maintain a constant pool size. If a new cluster has a similarity score of less than 0.3 with one or more pool clusters and lower energy than all of those pool structures, it will replace either the most similar pool cluster if the smallest score is less than 0.1 or the similar pool cluster with the highest energy if all scores are larger than 0.1 . The similarity examination maintains the structural diversity of the pool, which improves the convergence speed and the success rate. ${ }^{3}$ Following the update of pool, new parents are picked to reproduce next round of child clusters. This cycle continues until the genetic algorithm reaches a convergence criterion or the total number of clusters exceeds a maximum value. A schematic workflow summarizing this workflow is shown in Figure S1.

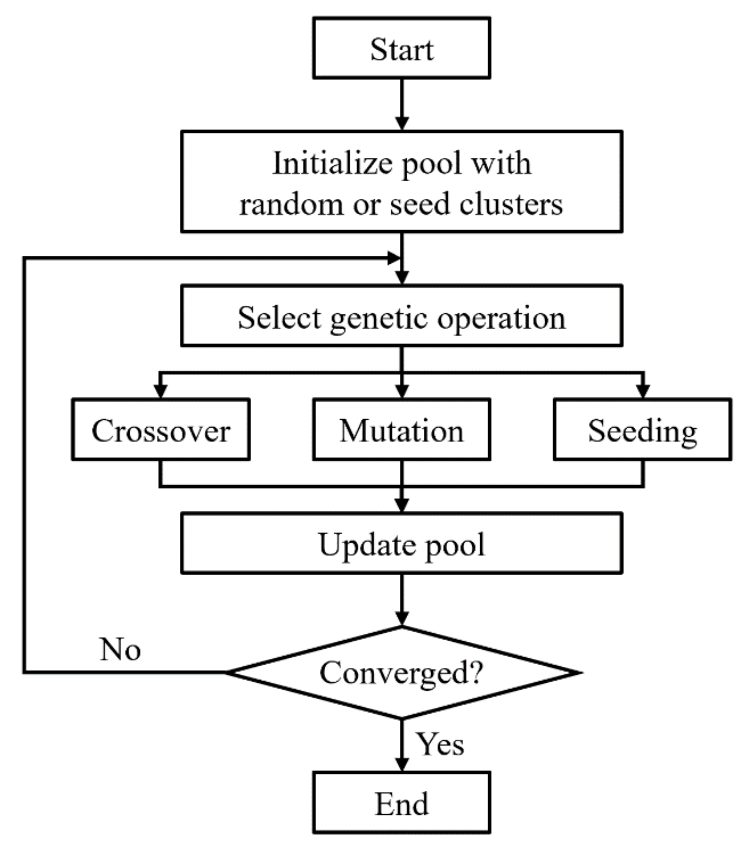

Figure S1. Schematic workflow for the primitive genetic algorithm without active learning acceleration.

\subsection{Convergence criteria and total running time}

We consider the GA to be converged when the pool becomes stagnant, which is defined here as occurring after the GA continuously generates 4000 or more clusters without discovering a cluster with lower energy than the current lowest value. For the calculations presented in the main text, GA_ALs were initially run for 3 days (1728 CPU-hours) for sizes 21 to 40 and for 6 days (3456 CPU-hours) for sizes 41 to 55 , followed with a check for stagnation. For the sizes in which the 
convergence criterion was not met, the GAs were resumed until convergence. GA_DFT, on the other hand, is computationally expensive and evaluating 4000 clusters or more can be prohibitively expensive for the sizes investigated in this work. To ensure a fair comparison with GA_ALs, GA_DFT calculations were run for at least the same number of CPU-hours as the corresponding GA_AL calculations. For clusters with 50 to 55 atoms, GA_DFT calculations were run for 21 days, much longer than GA_DFT calculations on small to medium-sized clusters, to reach similar energy levels as GA_AL calculations and to make comparisons meaningful. Table S1 summarizes computational time for all GA runs in this work. The computing center (MARCC) we used has a wall-time limit of 3 days for each individual job, so calculations were mostly performed for a multiple of 3 days. Some calculations that were very close to convergence (e.g., for sizes 39, 40, $47,55)$ were continued for only one or two days, which was sufficient to reach convergence.

Table S1. Computation time in days for all GA calculations in the main text.

\begin{tabular}{|c|c|c|c|c|c|c|c|}
\hline $\begin{array}{c}\text { Cluster } \\
\text { Size }\end{array}$ & $\begin{array}{l}\text { GA_AL } \\
\text { with } \\
\text { pre-trained } \\
\text { potentials }\end{array}$ & $\begin{array}{l}\text { GA_AL } \\
\text { without } \\
\text { pre-trained } \\
\text { potentials }\end{array}$ & GA_DFT & $\begin{array}{c}\text { Cluster } \\
\text { Size }\end{array}$ & $\begin{array}{l}\text { GA_AL } \\
\text { with } \\
\text { pre-trained } \\
\text { potentials }\end{array}$ & $\begin{array}{c}\text { GA_AL } \\
\text { without } \\
\text { pre-trained } \\
\text { potentials }\end{array}$ & GA_DFT \\
\hline 21 & -- & 3 & 3 & 39 & - & 5 & 5 \\
\hline 22 & -- & 3 & 3 & 40 & -- & 4 & 4 \\
\hline 23 & -- & 3 & 3 & 41 & 6 & -- & 6 \\
\hline 24 & -- & 3 & 3 & 42 & 6 & -- & 6 \\
\hline 25 & -- & 3 & 3 & 43 & 6 & -- & 6 \\
\hline 26 & -- & 3 & 3 & 44 & 6 & -- & 6 \\
\hline 27 & -- & 3 & 3 & 45 & 6 & -- & 6 \\
\hline 28 & -- & 3 & 3 & 46 & 6 & -- & 6 \\
\hline 29 & -- & 3 & 3 & 47 & 7 & -- & 7 \\
\hline 30 & -- & 3 & 3 & 48 & 6 & -- & 6 \\
\hline 31 & -- & 3 & 3 & 49 & 6 & -- & 6 \\
\hline 32 & -- & 3 & 3 & 50 & 6 & 6 & 21 \\
\hline 33 & -- & 3 & 3 & 51 & 6 & 9 & 21 \\
\hline 34 & -- & 3 & 3 & 52 & 6 & 6 & 21 \\
\hline 35 & -- & 3 & 3 & 53 & 6 & 11 & 21 \\
\hline 36 & -- & 3 & 3 & 54 & 12 & 17 & 21 \\
\hline 37 & -- & 3 & 3 & 55 & 7 & 9 & 21 \\
\hline 38 & -- & 3 & 3 & & & & \\
\hline
\end{tabular}

\subsection{GA input parameters}

Parameters controlling the GA workflow can affect the convergence speed. ${ }^{3,4}$ Figure S2 presents energy evolution plots of GA runs with varying ratios of mutated atoms in mutation operations and different inheritance ratios in crossover operations. The benchmarks were performed on clusters of sizes 54 and 55 with the mixed-size potentials labelled as "even" and "odd" in the main text. Since we are primarily concerned about the efficiency of GA operations instead of prediction 
accuracy, energies were only evaluated by MTP and the primitive GA workflow (Figure S1) was used (GA_MTP). All runs were stopped once the total number of clusters exceeded 5000. For both sizes, crossover is more efficient than both types of mutation operations in terms of the rate of convergence to low-energy structures. Among the two types of mutation operations, rotation is better than random moves. Within crossover benchmarks, the random method for calculating inheritance ratios of parents outperformed the even method, while it was close to the weighted method. As for mutation, the lowest energy for both sizes and both types of mutation operations was achieved when $10 \%$ of the atoms were moved, although this approach did not have the fastest convergence speed (Figure S1a and Figure S1f). These findings suggest that adjustments to a small number of atoms are more efficient in minimizing the energy, while operations involve large number of atoms often stagnate at elevated energy levels. This can be explained from the fact that often only a small fragment of atoms is out of place compared with the lowest-energy structure in the late stages of a GA optimization. Relocating only a few atoms is more likely to put dislocated atoms into the right positions than simultaneously displacing a large chunk of atoms from a structure which is already close to the lowest-energy isomer.

54

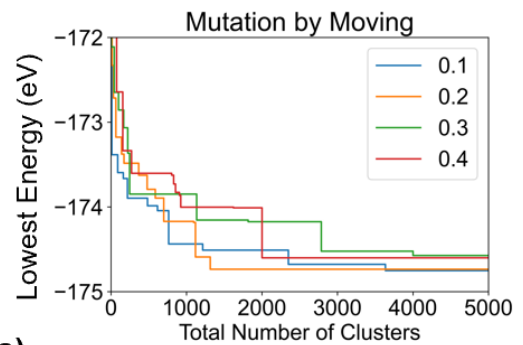

a)

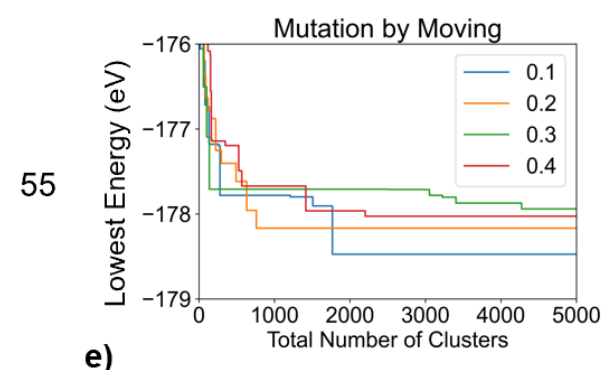

e)

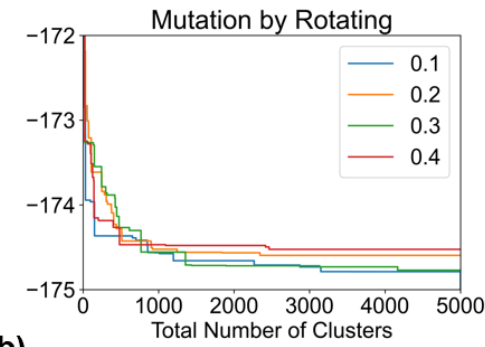

b)

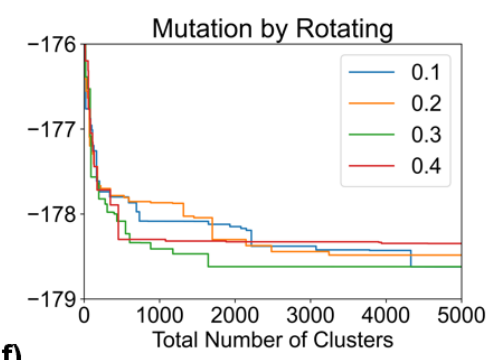

f)

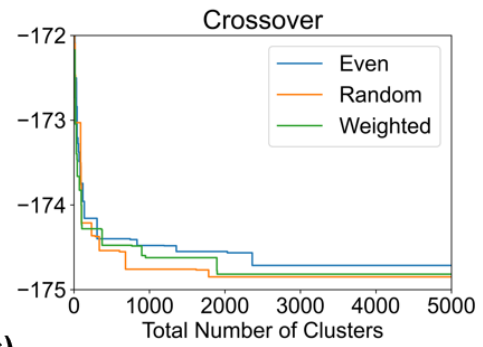

c)

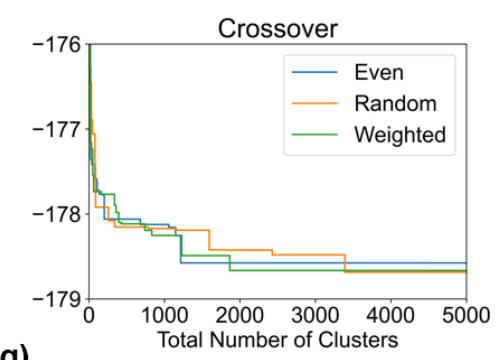

Figure S2. Energy evolution plots of GA_MTP for different GA operations and controlling parameters. a) and e) mutation by randomly displacing atoms with varying ratios of mutated atoms. b) and f) mutation by randomly rotating atoms around center of mass with different ratios of mutated atoms. c) and g) crossover with various calculation methods for ratios of inherited atoms from parents. The plots on first row uses the "even" potential discussed in main text on size 54, while those on the second row uses the "odd" potential on size 55.

For all GA runs in the main text, both move and rotation were used in mutation, and in each mutation operation a maximum $10 \%$ of atoms were allowed to be displaced. For crossover, both random and weighted methods were used with an equal probability. Moreover, since crossover is more efficient than mutation, the frequency of applying mutation in cluster generation was suppressed by capping the fraction of pool clusters created by mutation at $0.2 .^{5}$ In other words, if 
$20 \%$ or more pool clusters are produced by mutation, the subsequent GA operations will always be crossover until the percentage drops below $20 \%$. The pool size was kept at 25 , as it offers a good balance between convergence speed and success rate of identifying the lowest-energy cluster. ${ }^{3,4}$

\subsection{GA_AL input parameters}

When initializing GA_AL with pre-trained potentials, it is beneficial to switch off retraining at the beginning of the search, to let GA fully explore the potential energy surfaces of pre-trained potentials. We call the parameter controlling the number of clusters that can be generated before retraining is turned on "preprocessing". During the preprocessing period, extrapolating structures are discarded, and new clusters are generated until all MTP-relaxed configurations are interpolating. This ensures the GA search stays in interpolating region of the pre-trained potential in configuration space. As Figure S3 shows, the lowest energies in the first retraining were achieved when preprocessing was between about 5000 and 7500 clusters. Exceeding this range, artificial local minima start to populate the pool and drive up the lowest energy. To accelerate GA_AL and minimize time spent in the preprocessing stage, we used a preprocessing value of 5000 in all GA_AL runs in this work initialized with pre-trained potentials.

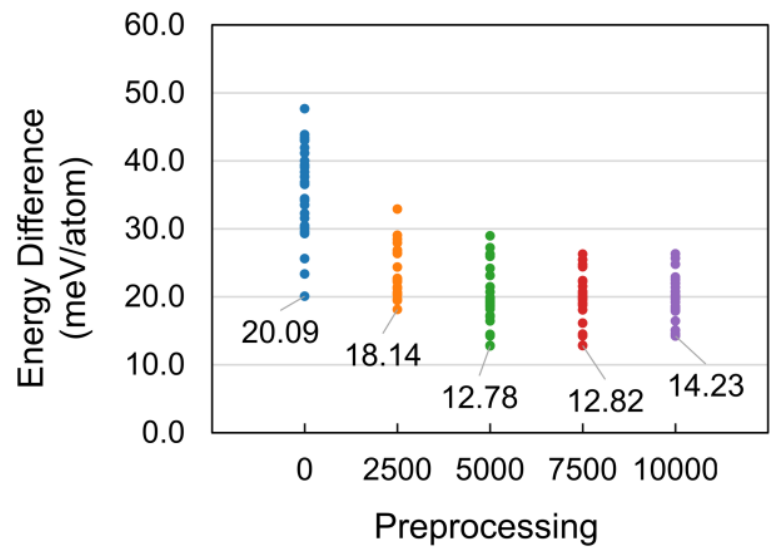

Figure S3. DFT energy distribution of pool clusters in the first retraining after preprocessing number of clusters has been generated in GA_AL. Tests were performed on clusters with 55 atoms and GA_AL runs were initialized with the mixed-size potential trained on odd number of clusters ("odd" potential in Figure 3 of the main text). Energies are reported relative to the energy of the lowest-energy cluster with 55 atoms. ${ }^{19}$ 


\section{Hyperparameter selection for MTP}

\subsection{Data selection and training}

To identify a good set of hyperparameters for training MTP potentials, we used Al clusters with 24 atoms as a model system and tested various combinations of hyperparameters, including potential complexity (defined by the level of moments, $l e v_{\max }$, of a MTP potential) ${ }^{20}$ the amount of training data, and relative weights for force components and energies. We first performed GA_DFT searches on Al clusters with 24 atoms and then used the same diversity-based strategy as used in the preparation of mixed-size potentials (see Methods section of the main text) to select dissimilar configurations from the GA_DFT runs. We then randomly separated all DFT calculations into training and validation groups by keeping the ratio of the total number of dissimilar ionic steps in the training group to that of the validation group at approximately $3: 1$. To ensure structural dissimilarity of clusters in the training dataset from those in the validation set, we assign all ionic steps belonging to each relaxation trajectory to either the training or validation groups. This increases the likelihood that the validation results reliably reflect the accuracy of MTP potentials in areas of the configuration space that are not well-represented by the training data.

To assess the effect of training set size on potential accuracy, we used the same energy-based strategy as used for the preparation of mixed-size potentials (see Methods of the main text) to collect training datasets from the training group. We evaluated training sets containing 200, 400, $600,800,1000,1500,2000,2500$, and 3000 structures. For the validation dataset, we selected the 1000 lowest-energy ionic steps from the DFT calculations in the validation group, as the accuracy on low-lying areas of potential energy surfaces matters the most in ground state structure search.

The maximum number of iterations in Broyden-Fletcher-Goldfarb-Shanno algorithm ${ }^{21-24}$ for training the potentials was set to 5000 , which is 5 times larger than the default value of $1000 .^{20}$ Only trainings using small relative force weights less than 0.001 for potentials with levels of moments of 20, 22 and 24 stopped because of reaching this limit. We expect none of these trainings will affect the conclusions we draw from the set of benchmarks. None of the trainings in Figure 1 of the main text were terminated due to hitting the limit.

\subsection{Analysis of various combinations of parameters}

The main text presents the benchmarks that are most relevant to the hyperparameters that we used in GA_AL runs. Here, we give a more comprehensive analysis of all parameter combinations that we tested. They all show similar trends as the ones shown in the main text. A force weight that is $1 / 1000$ of the energy weight offers the best balance between prediction accuracies on force components and energies (Figure S4). Both force and energy root-mean-square errors (RMSEs) decrease as potential complexity rises (Figure S5). However, the training cost increases dramatically with both training data size and potential complexity (Figure S6). A somewhat surprising finding is that larger force weights took less time to train than smaller ones. A possible explanation is that the potential energy surface for atomic clusters is very rough, making it difficult to reproduce energies with a high accuracy, whereas there was less variation in the forces for nearly-relaxed structures. The potentials with $l e v_{\max }$ of 10 and 12 see a steady increase of energy 
validation RMSEs as the training set grows. Potentials with $l e v_{\max }$ larger than 14 have relatively flat force and energy RMSEs after the training set size exceeds 2000, but there can be significant variation with respect to training set size for training sets with fewer than 1000 structures (Figure S7).

a)
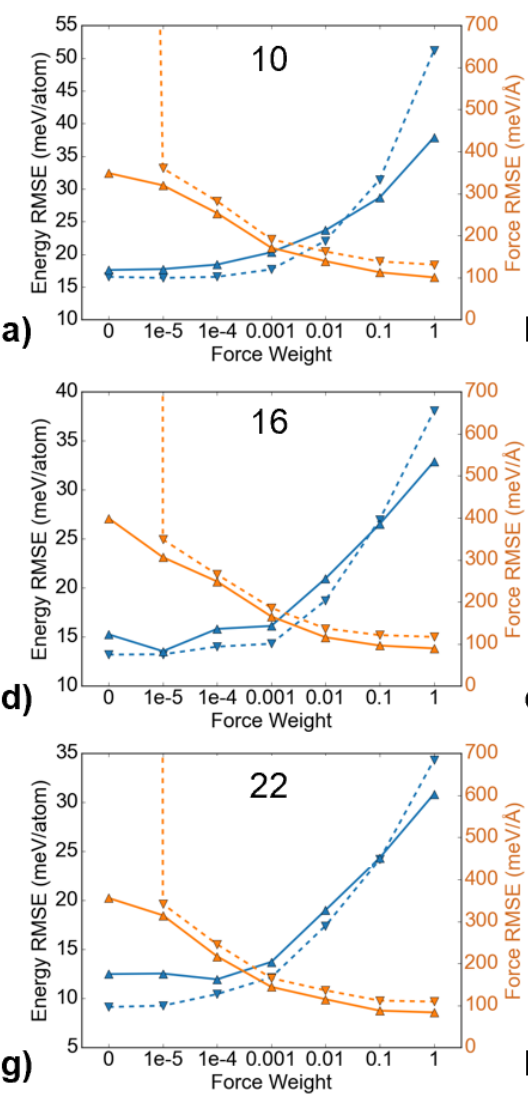
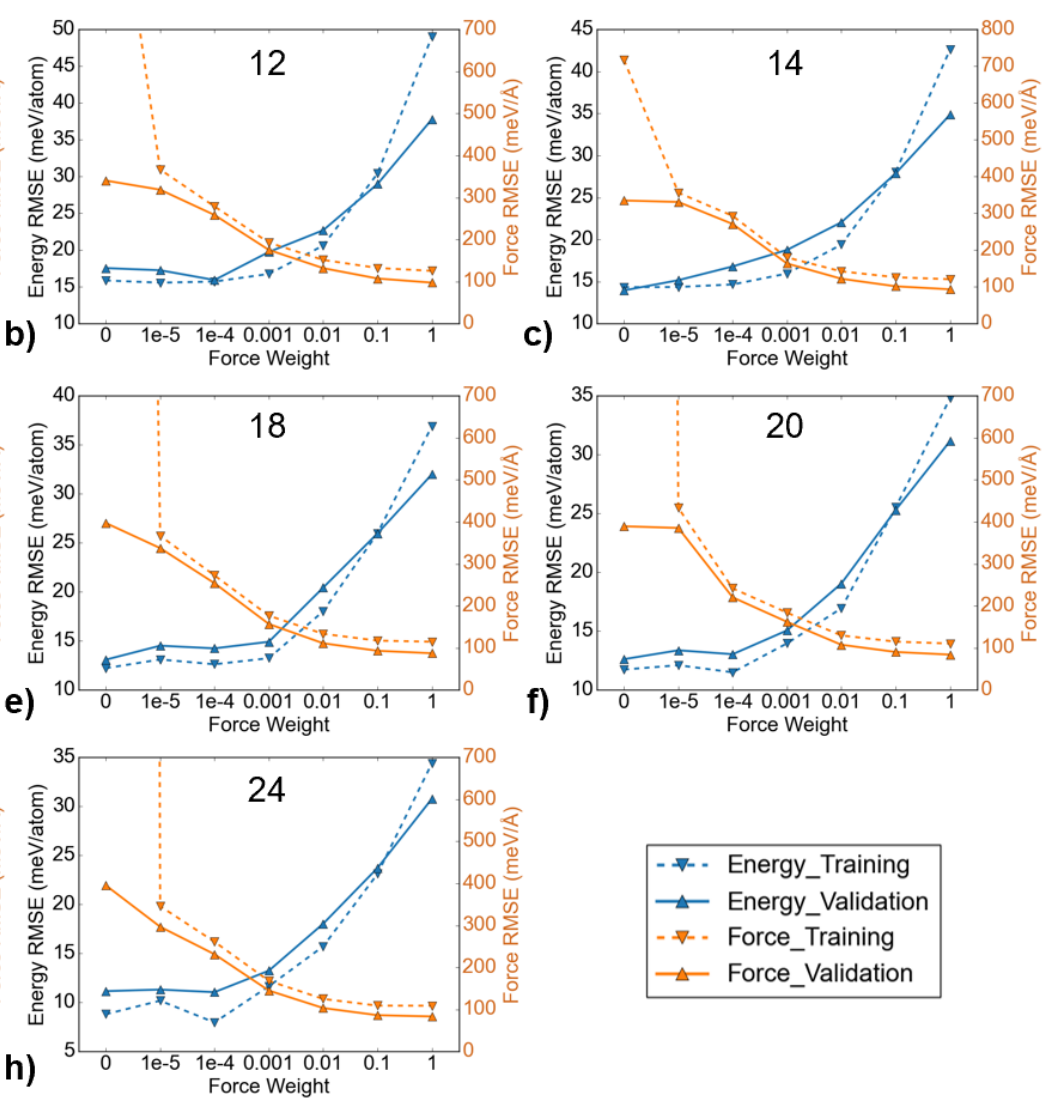

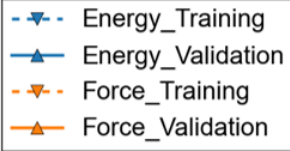

Figure S4. Training and validation errors against relative force weights to energy across different potential complexities. Training and validation RMSEs are marked by dashed and solid lines, respectively. In all benchmarks, the energy weight was set to 1 and a training set of 3000 structures was used. 
a)
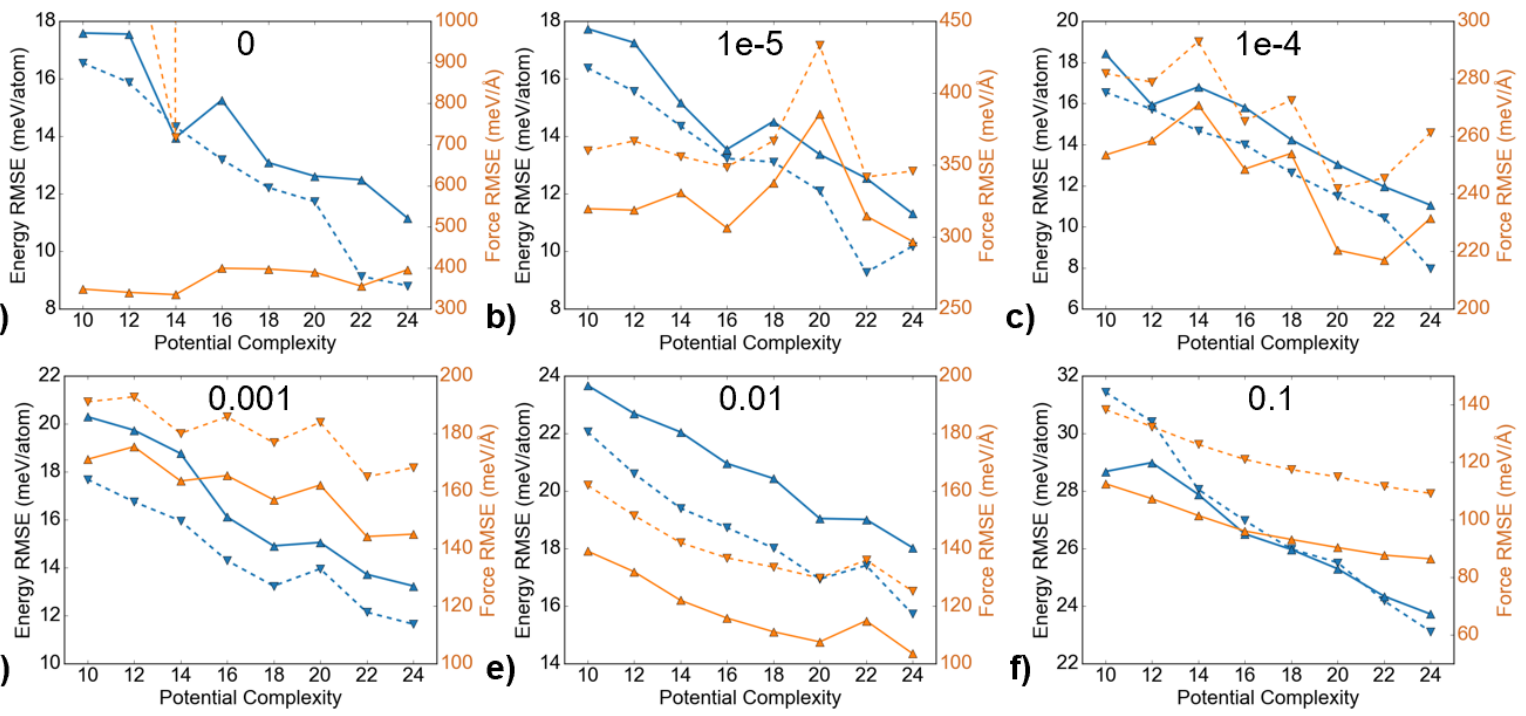

g)

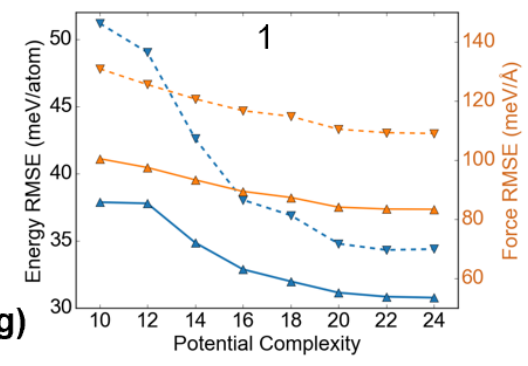

$$
\begin{aligned}
& -\forall-\text { Energy_Training } \\
& \rightarrow-\text { Energy_Validation } \\
& -\nabla-\text { - Force_Training } \\
& \rightarrow-\text { Force_Validation }
\end{aligned}
$$

Figure S5. Training and validation errors against increasing potential complexities across varying force weights relative to energies. For all benchmarks, the energy weight was kept at 1 and a training set of 3000 structures was used. 


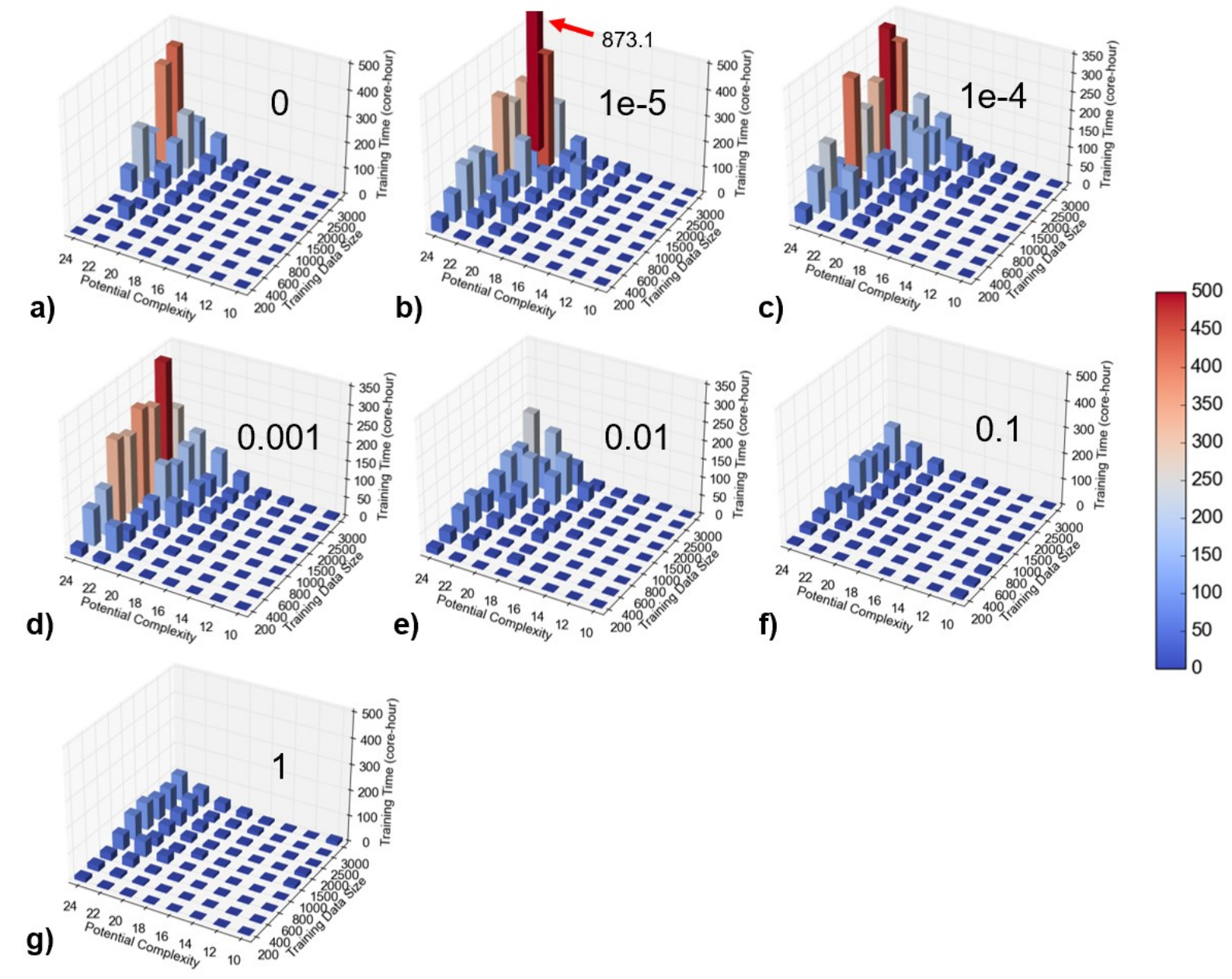

Figure S6. Training time plots in unit of core-hour against potential complexity and training set size for increasing force weights relative to energy. 


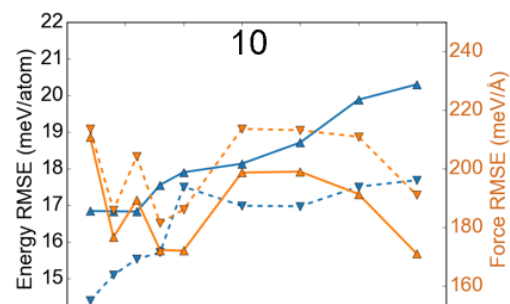

a) ${ }^{14} \begin{array}{r}500 \quad 1000 \quad 150020002500 \quad 3000 \\ \text { Training Data Size }\end{array}$
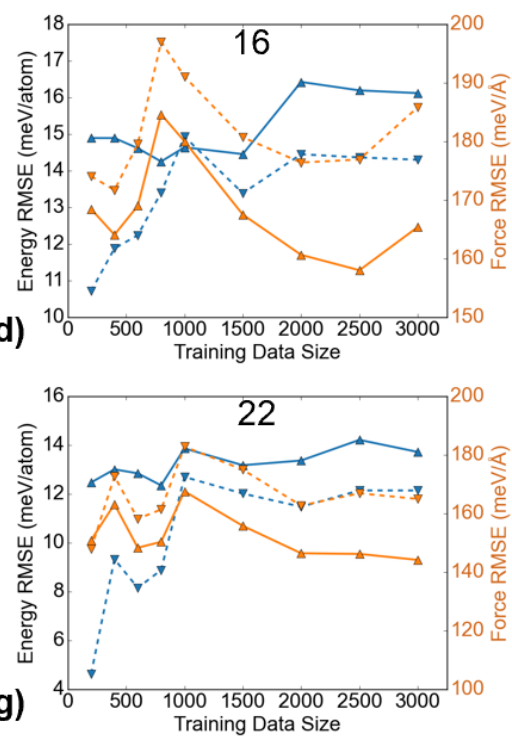

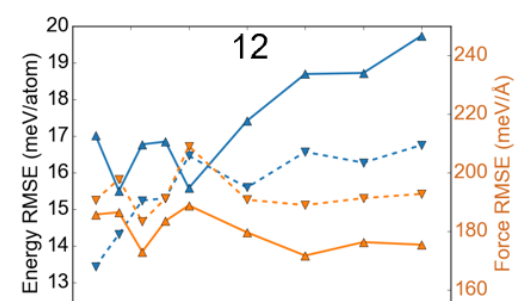

b) ${ }^{12}\left[\begin{array}{cc}500 \quad 1000150020002500 \quad 3000 \\ \text { Training Data Size }\end{array}\right.$
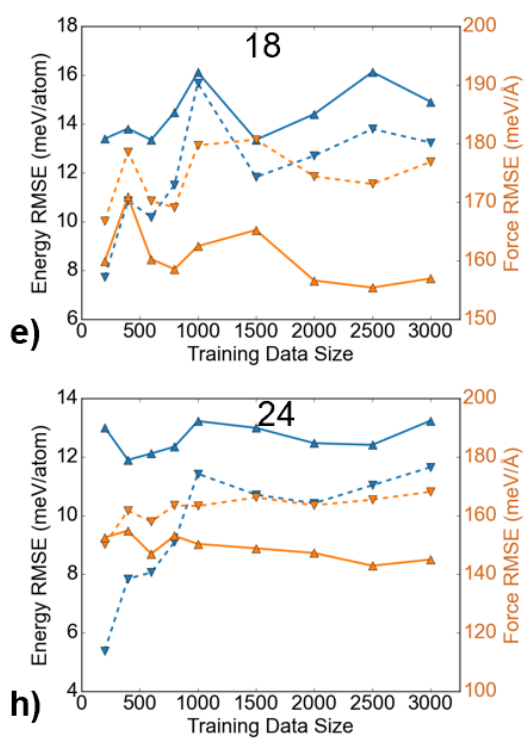
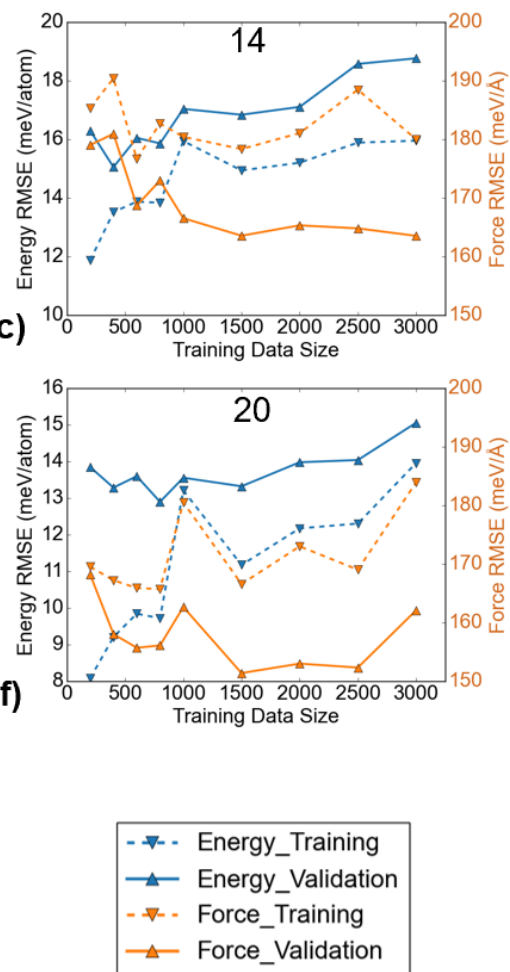

Figure S7. Training and validation errors against size of training set across different potential complexity. In all benchmarks, relative weight of force components to energies was kept at $1 / 1000$. 


\section{Validation of pre-trained potentials}

\subsection{Validation dataset for pre-trained potentials}

The validation data are collected from GA_DFT runs on clusters with 50-55 atoms. The same diversity-based method as the one used in preparation of the training data of the pre-trained potentials (see Methods section of the main text) was used to select a structurally diverse set of structures. Table S2 lists the total number of validation structures for each cluster size.

Table S2. Total number of structures in validations sets of clusters with $\mathbf{5 0}$ to 55 atoms.

\begin{tabular}{cc}
\hline \hline Cluster Size & $\begin{array}{c}\text { Number of Validation } \\
\text { Structures }\end{array}$ \\
\hline 50 & 3840 \\
51 & 2946 \\
52 & 3653 \\
53 & 2581 \\
54 & 3578 \\
55 & 2604 \\
\hline \hline
\end{tabular}

\subsection{Energy parity plots for pre-trained potentials}

Figure S8 shows parity plots of MTP predicted energies against DFT calculated energies for the same set of validations presented in Figure 3 of the main text. The single-size potentials trained solely on small clusters of $21,22,25$ and 26 atoms consistently underestimated low-lying structures of large sizes by a relatively large error, while the underestimation was smaller for potentials trained on clusters of 29 to 38 atoms. The mixed-size potentials exhibit a better fit for all validation sets, indicating enhanced transferability compared with single-size potentials. 
a)

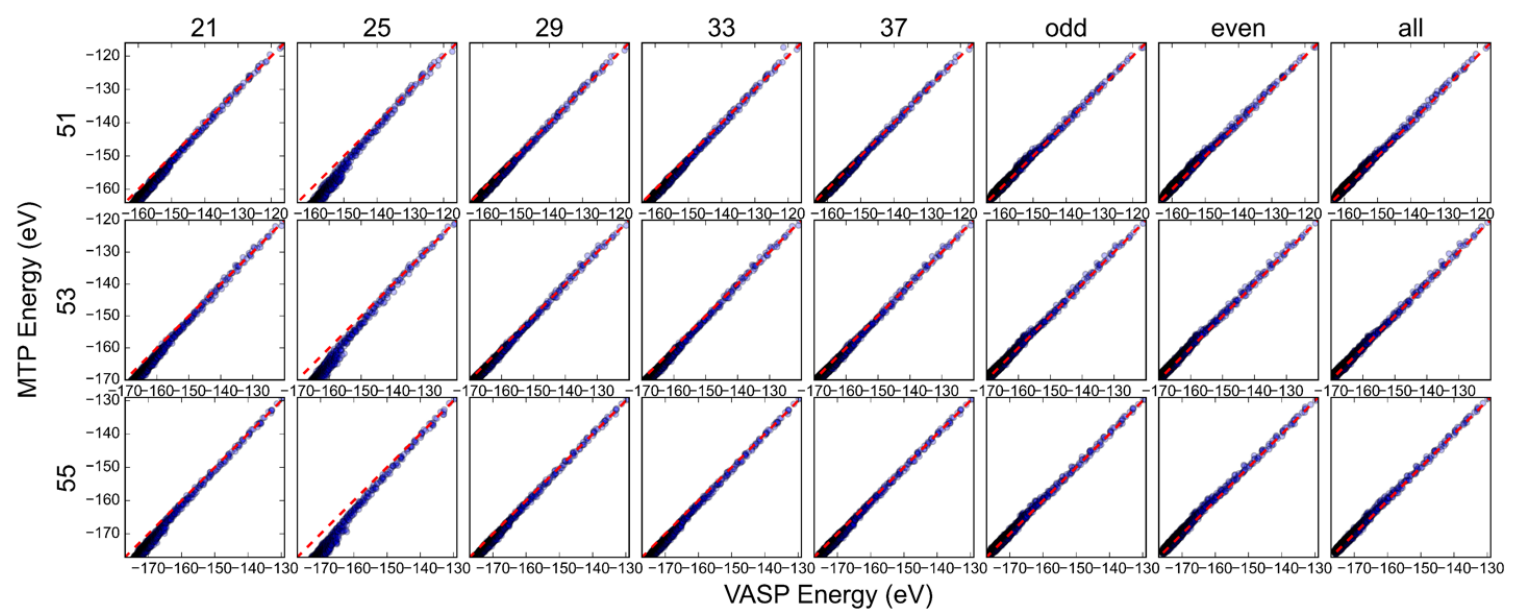

b)

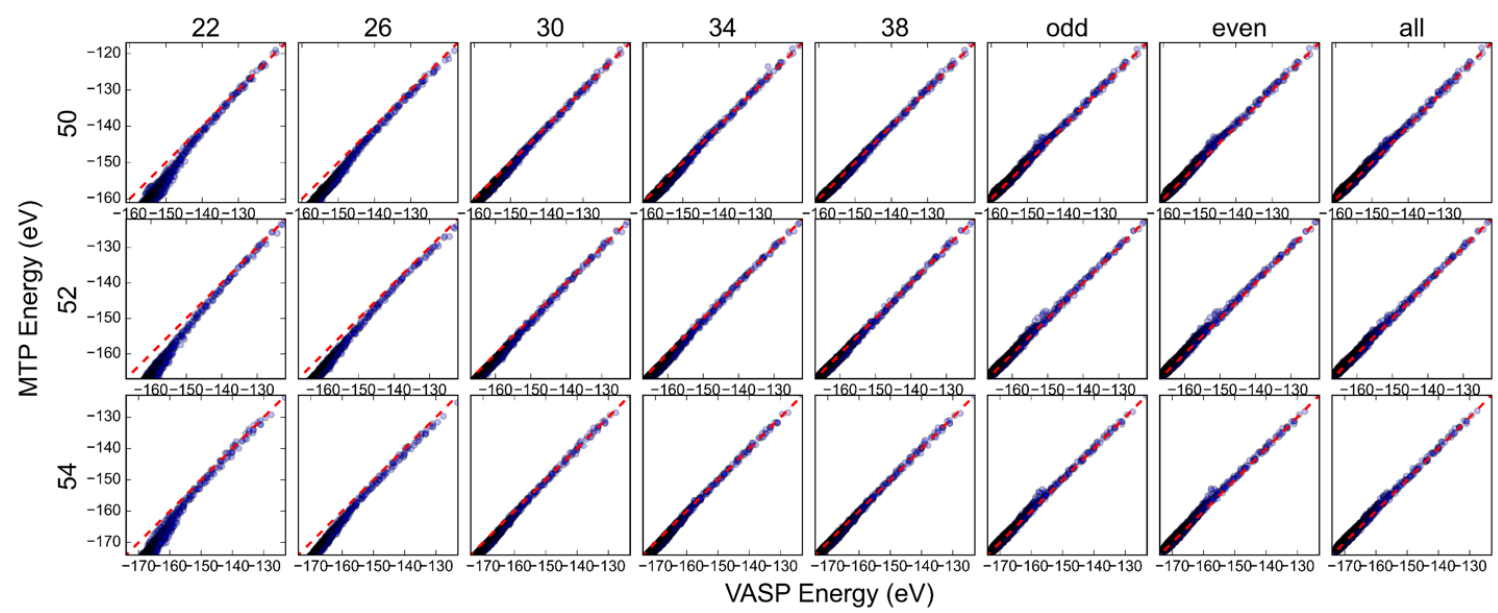

Figure S8. Energy scatter plots for validation of single-size and mixed-size potentials on large clusters with 50-55 atoms. Plots are grouped by parity of number of atoms into a) an odd group, and $\mathbf{b )}$ an even group.

\subsection{Validation of mixed-size potentials on lowest-energy clusters with 50 to 55 atoms}

Since we are primarily concerned with potential accuracies on low-energy structures, the singlesize and mixed-size potentials were also validated on the lowest-energy clusters of sizes with 5055 atoms, which were collected from GA_AL and GA_DFT runs of this work, and from the literature. ${ }^{19}$ The lowest-energy clusters were grouped into a set of even-sized clusters and a set of odd-sized clusters.

Figure S9 displays the average energy errors of MTP predictions, and the average extrapolation grades of the lowest-energy clusters in different potentials. The errors follow a similar trend as the energy validation RMSEs in Figure $3 \mathrm{a}$ and Figure $3 \mathrm{~b}$ of the main text. Prediction errors are larger for single-size potentials trained with clusters with small number of atoms, and these errors decrease gradually as the size of the clusters in the training set increases. Mixed-size potentials have consistently lower errors than any single-size potentials. Potentials trained with clusters of both even and odd number of atoms, hence different magnetic moments, do not greatly deteriorate 
accuracy. Surprisingly, the odd potential gave a better prediction than the even potential for the lowest-energy clusters with even number of atoms, which is also the case for the even potential on lowest-energy clusters with odd number of atoms. This suggests the gap between potential energy surfaces with different magnetic moments is not larger than the uncertainty of the moment tensor potentials. Mixing training structures of different magnetic moments results in an average effect: errors for the potential trained on clusters with both even and odd number of atoms are between the errors of the potentials trained on clusters with only even or odd numbers of atoms.

The trend in extrapolation grades provides additional evidence showing that mixing training data across a range of number of atoms can systematically enhance transferability to clusters of sizes that were not in the training set. For all six of the lowest-energy clusters, extrapolation grades of single-size potentials get smaller as the number of atoms in the training structures grows. Nearly all of them are extrapolating (i.e., the extrapolation grade is above 1), with the exception of the potential trained on clusters with 37 atoms, which has an extrapolation grade of 0.95 . However, for the even and odd potentials, these lowest-energy clusters are interpolating, and energy predictions are in good agreement of DFT calculations, with errors well within potential uncertainties as shown in Figure 3 of the main text. Since the goal of the structure search is to discover unknown low energy configurations, high prediction accuracy on low-lying regions of potential energy surface is vital. Thus, validations on the lowest-energy clusters demonstrate that our strategy of constructing mixed-size potentials transferrable to clusters with number of atoms not present in the training data can be very helpful in improving the efficiency and success rate of the genetic algorithm.
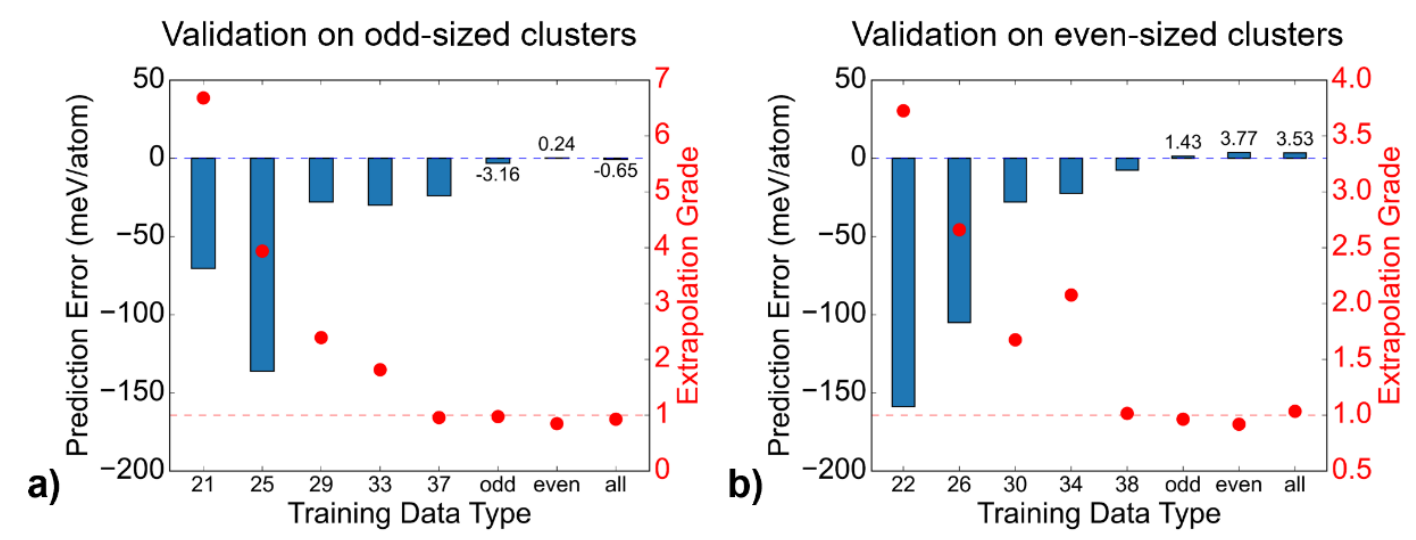

Figure S9. Validations of different potentials on the lowest-energy clusters with a) 50, 52, 54 atoms, and b) 51, 53, 55 atoms. Labels on $x$-axis share the same meaning as those in Figure 3 of the main text. The primary y-axis on the left and blue bars indicate average energy prediction errors for the lowest-energy clusters. The secondary y-axis on the right and the red dots represent the average extrapolation grades of the lowest-energy clusters in corresponding potentials. 


\section{Magnetic moments of low-energy clusters}

We assembled in another work a database comprising low-energy clusters of 55 elements in the first five periods of the periodic table. ${ }^{25}$ For aluminum clusters, the even-sized structures having the lowest energies are always non-magnetic when they have more than 16 atoms, while the lowlying odd-sized structures always have a net magnetic moment within $0.002 \mu_{B}$ of $1 \mu_{B}$. Figure S10 displays the magnetic moments of the lowest-energy clusters in the database at the time we submitted this manuscript.

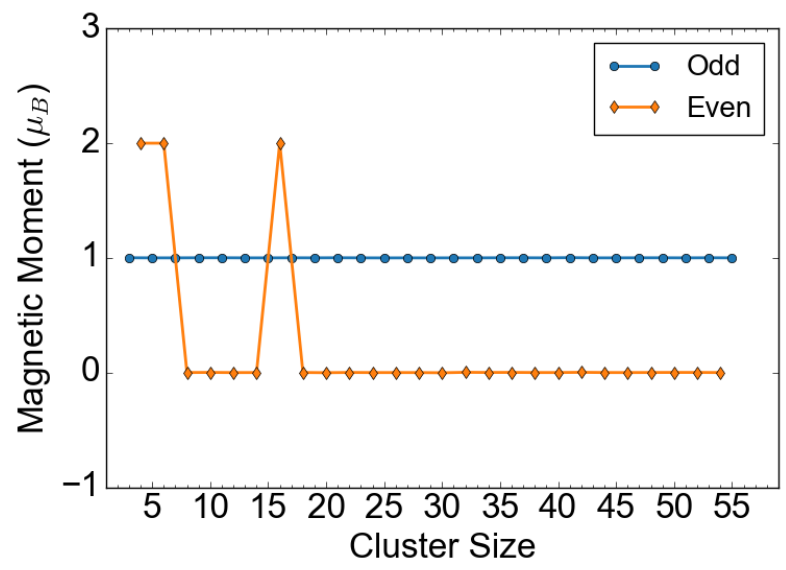

Figure S10. Magnetic moments of the lowest-energy aluminum clusters in the database with 3 to 55 atoms. 


\section{Performance analysis of GA_AL and GA_DFT}

\subsection{Energy evolution plots for GA_AL and GA_DFT on clusters with 21 to 49 atoms}

Figure S11 and Figure S12 present the energies of the lowest-energy clusters as GA_AL and GA_DFT proceeded, for clusters with 21 to 49 atoms. Acceleration ratios are computed by dividing the earliest time that GA_AL discovers clusters with equal or lower energy than the energy of the lowest-energy cluster found by GA_DFT over the earliest time that GA_DFT finds that lowest energy cluster. These energy values are marked by black dashed lines in the energy evolution plots. The time points at which the step lines cross the dashed lines indicate the times used in acceleration ratio measurement and are marked by black crosses. In cases where GA_AL did not discover equivalent or better clusters (size 29 and 45), the acceleration ratios are 0 , and the dashed lines and crosses are not drawn. 

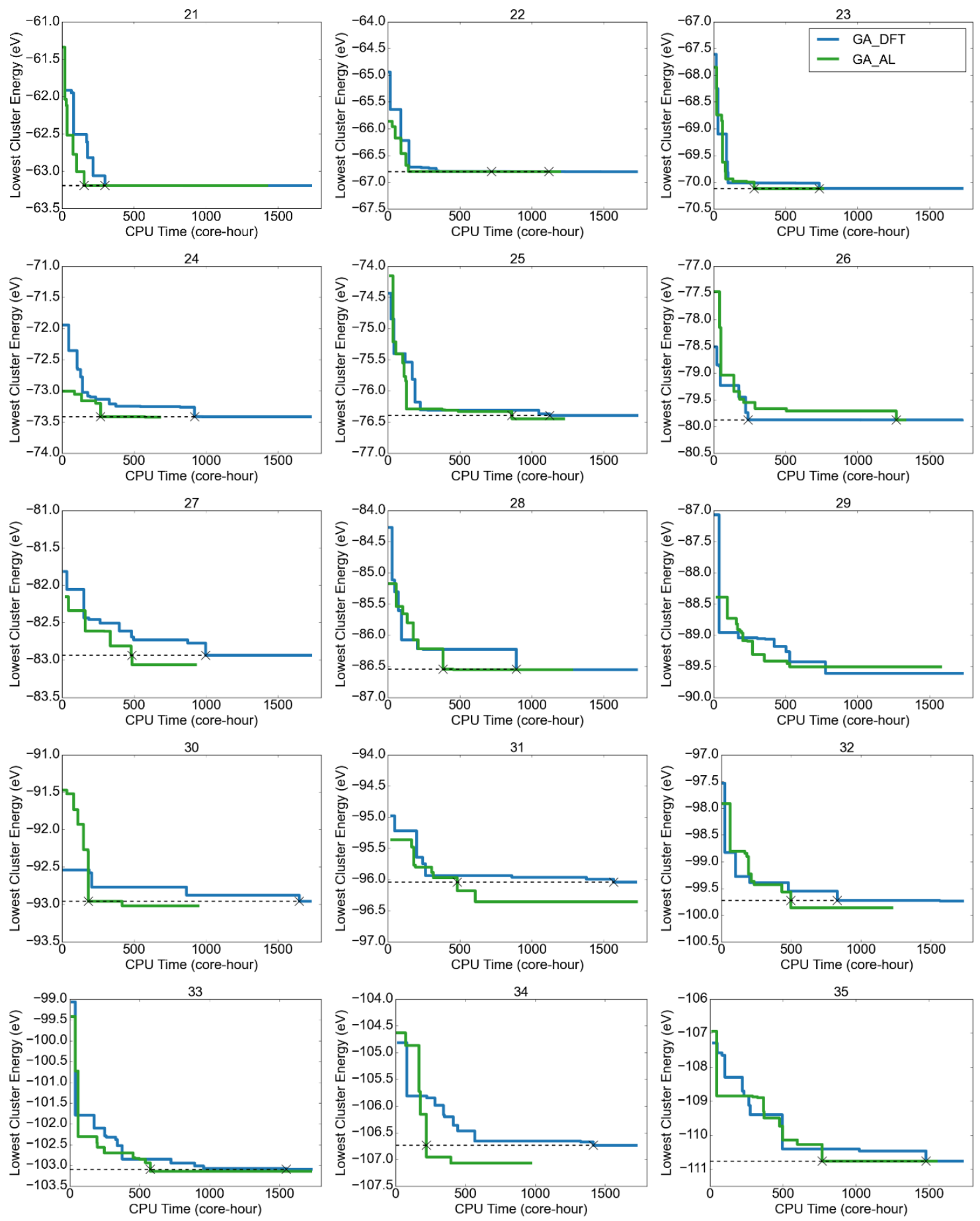

Figure S11. Energy evolution plots of GA runs for clusters with 21-35 atoms. The blue, and green step lines represent GA_DFT and GA_AL initialized with untrained potentials. The black dashed lines mark the energy levels that are used in determining acceleration ratios. The black crosses mark the earliest time at which each method reached the corresponding energy levels. These times are used in computing the acceleration ratios. For size 29 in which GA_AL did not find clusters with energies as low as the lowest-energy cluster in GA_DFT, the ratio is set to 0 and no crosses were marked. 

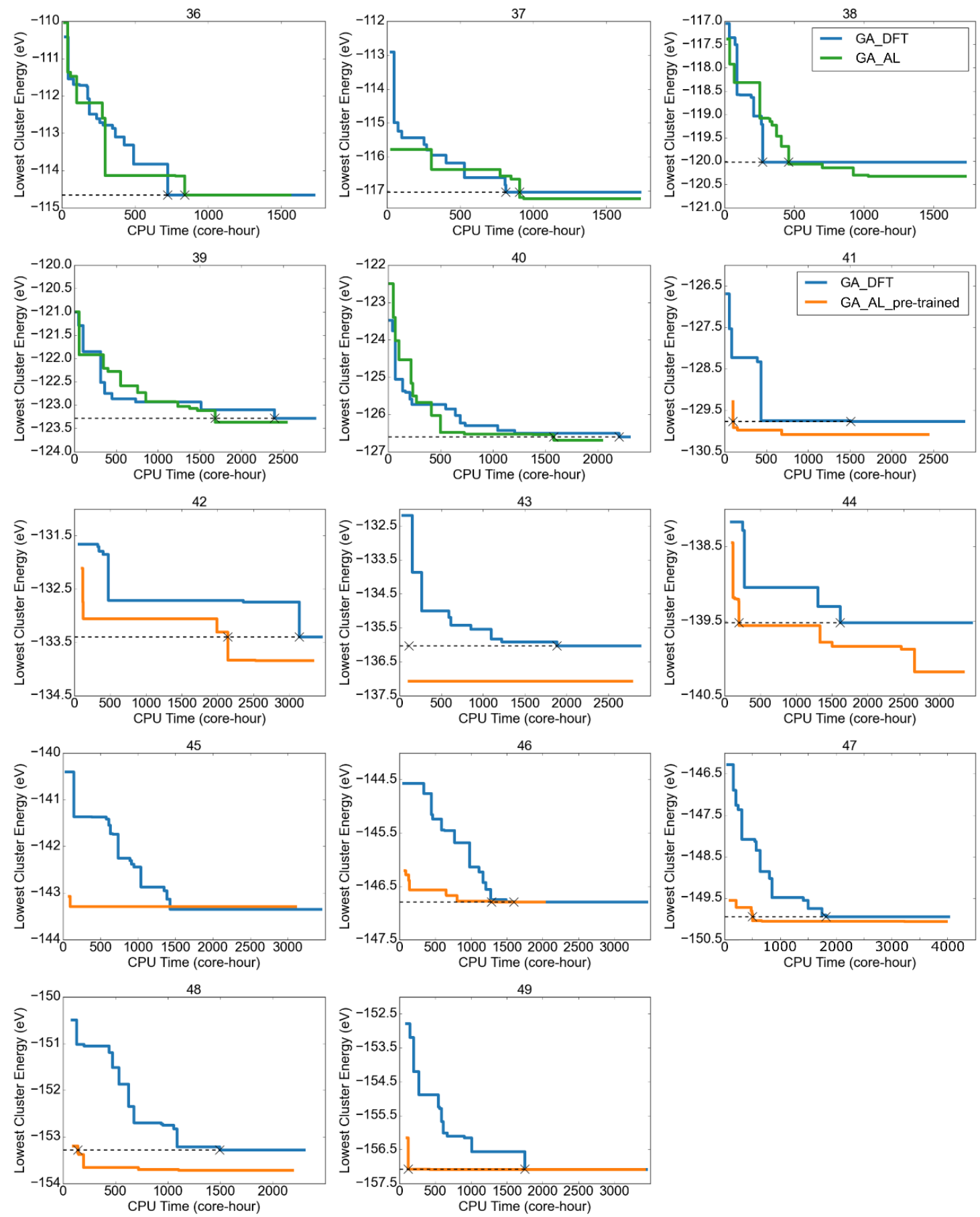

Figure S12. Energy evolution plots of GA runs for clusters with 36 to 49 atoms. The blue lines represent GA_DFT, green lines represent GA_AL initialized with untrained potentials, and orange lines represent GA_AL initialized with pre-trained transferable potentials. The black dashed lines mark the energy levels that are used in determining acceleration ratios. The black crosses mark the earliest time at which each method reached the corresponding energy levels. These times are used in computing the acceleration ratios. For size 45 in which GA_AL did not find clusters with energies as low as the lowest-energy cluster in GA_DFT, the ratio is set to 0 and no crosses were marked. 


\subsection{Performance comparison of GA_AL with and without pre-trained potentials}

We performed both GA_AL initialized with pre-trained potentials and GA_AL initialized with untrained potentials on clusters of 50 to 55 atoms. The latter approach took longer time than the former to find the same lowest-energy clusters (see Figure 4a of the main text). Figure S13 shows the acceleration ratios and differences of lowest energies compared to GA_DFT for both approaches. Averaged over cluster sizes of 50 to 55, GA_AL initialized with untrained potentials had an acceleration ratio of 3.37, compared with the much larger ratio of 18.23 for GA_AL initialized with the mixed-size potentials.

a)

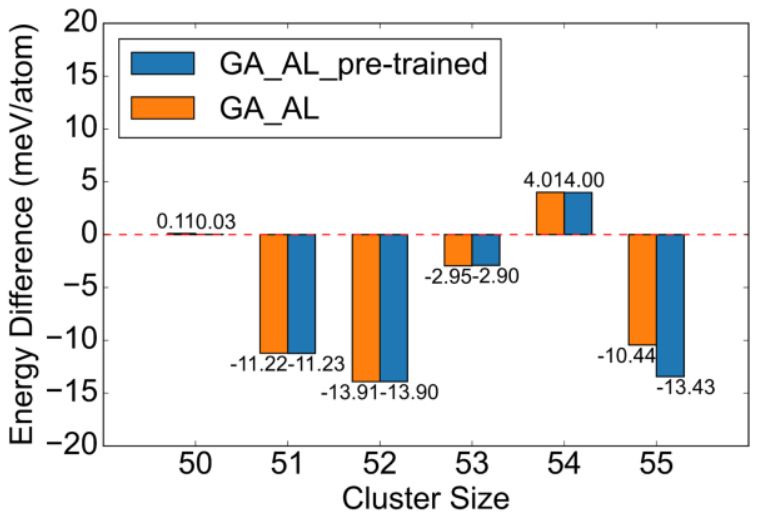

b)

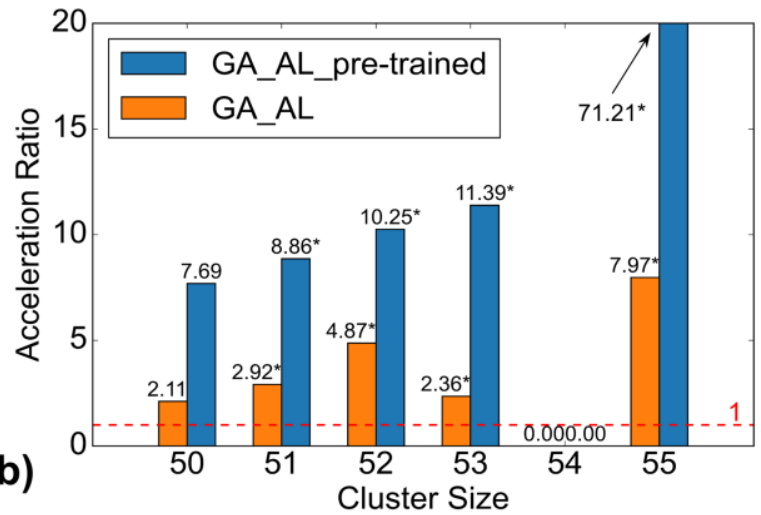

Figure S13. Comparison of performances between GA_AL initialized with pre-trained mixed-size potentials and with untrained potentials on clusters with 50 to 55 atoms. Acceleration ratios and energy differences of lowest-energy clusters are calculated in the same way as Figure $2 \mathrm{~b}$ and Figure $4 \mathrm{c}$ of the main text. 


\section{Identification of new lowest-energy clusters}

In the main text, low-energy clusters were collected from three sources: GA_AL runs, GA_DFT runs and literature. To determine whether we have identified a new lowest-energy structure for a cluster size and to compare performance between the GA_AL and GA_DFT algorithms, the lowest-energy clusters found from different sources are compared both in total energies and similarity. A cluster is said to be a new lower-energy cluster than another if it is lower in total energy by at least $1 \mathrm{meV}$ /atom and the similarity score between the two clusters is larger than 0.3 . If a cluster has an energy difference smaller than $1 \mathrm{meV} /$ atom but is structurally dissimilar to another cluster, we count it as an "energetically similar" cluster. For the borderline cases where a cluster is lower in energy by more than $1 \mathrm{meV} /$ atom, but the similarity score between the two clusters is slightly less than 0.3 , we manually checked the structures to determine whether they are equivalent or not. These scenarios are summarized in Table S3.

Table S3. Summary of comparison results between clusters by total energy and structural similarity.

\begin{tabular}{|c|c|c|c|}
\hline \multirow{2}{*}{} & \multicolumn{2}{|c|}{ Similarity Comparison } \\
\cline { 3 - 4 } & Similarity Score $>0.3$ & Similarity Score $\leq 0.3$ \\
\hline \multirow{2}{*}{$\begin{array}{c}\text { Energy } \\
\text { Comparison }\end{array}$} & $\Delta E>1 \mathrm{meV} /$ atom & New, lower-energy & Visually check \\
\cline { 2 - 4 } & $\Delta E \leq 1 \mathrm{meV} /$ atom & Energetically similar & Equivalent \\
\hline
\end{tabular}

The same comparison methods are used in deciding whether a GA search (GA_DFT or GA_AL) has identified a new lowest-energy cluster compared with those reported in the literature ${ }^{19,26-28}$ and in comparing between clusters found by the GA_AL and GA_DFT algorithms. Cluster sizes for which there is a cluster that is energetically similar to the current lowest-energy cluster are considered to have multiple lowest-energy isomers. 
Table S4 listed the total energies and the similarity scores for lowest-energy clusters identified in GA_AL and GA_DFT runs of this work, and the ones reported in literature. The total energies are reported relative to the energies of the clusters found in GA_AL runs.

Three cluster sizes, 22, 33 and 46, requires additional examination. GA_DFT identified an isomer for clusters with 22 atoms that is energetically similar to the lowest-energy cluster reported in literature ${ }^{27}$ and found by GA_AL. For size 33, although the two low-energy clusters from GA_DFT and GA_AL have an energy difference larger than $1 \mathrm{meV} /$ atom, the similarity score and visual inspection (Figure S14) show that they are equivalent. For size 46, the lowest-energy cluster reported in literature has a similarity score with the ones found in GA_AL and GA_DFT that is less than 0.3 , but the energy difference and visual inspection show the structures are not equivalent. Therefore, the genetic algorithm is considered to have identified new lowest-energy clusters for sizes 22 and 46, but only confirmed the literature-reported cluster for size 33.

GA_AL identified new lowest-energy clusters for 23 sizes out of the total 35 sizes studied in this work. GA_AL runs and GA_DFT runs together identified 25 new lowest-energy clusters (26 if counting the energetically similar cluster for size 22) and confirmed the literature-reported lowestenergy clusters for 8 sizes. Overall, the GA runs of this work only missed the lowest-energy literature structures for clusters with 32 and 55 atoms. It is likely that these clusters could have been found more quickly if as seeing operation was used, given the similarity between the lowestenergy clusters of these two sizes to the clusters with close number of atoms (see Figure S15).

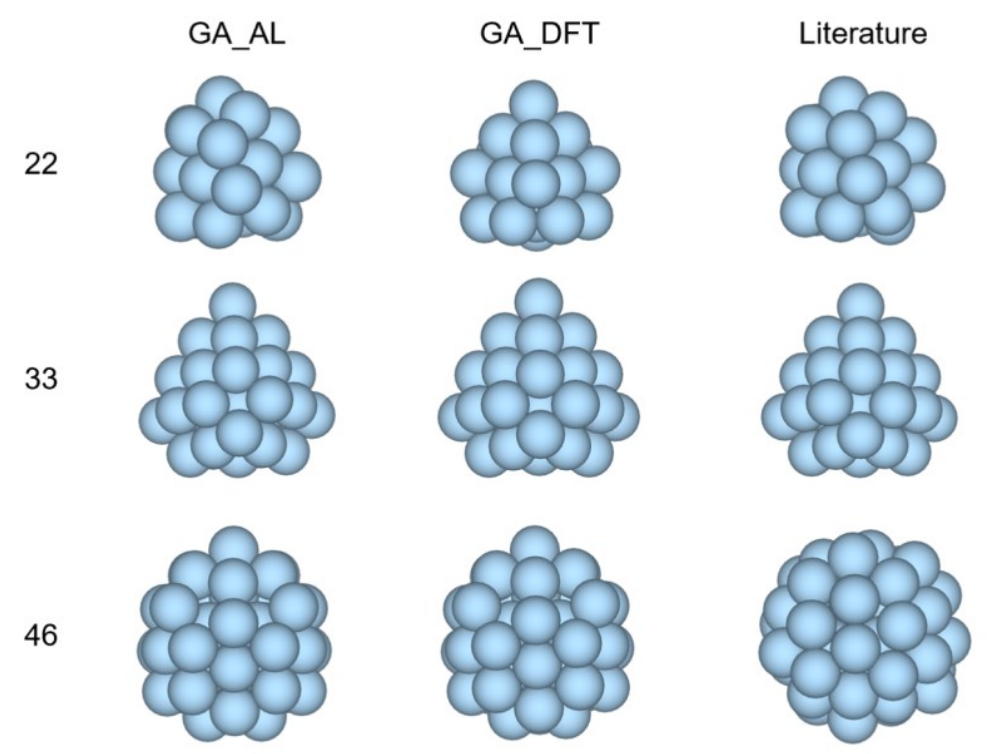

Figure S14. Lowest-energy clusters from GA_AL, GA_DFT and the literature ${ }^{27,28}$ for clusters with 22,33 , and 46 atoms. 
Table S4. Total energy differences and similarity scores between lowest-energy clusters found in GA_AL and GA_DFT runs of this work, and the ones reported in literature. "Lit." is the abbreviation for "Literature". Energy differences are reported in meV/atom relative to the total energies of lowest-energy clusters from GA_AL (include GA_AL initialized with untrained potentials or pre-trained potentials). Similarity scores are calculated using the same method reported in Methods section of the main text. The origins of the final lowest-energy clusters are listed in the last column. Three cluster sizes that display large energy differences but small similarity scores or small energy differences but large similarity scores are marked with asterisks and are discussed in detail in Section 6.

\begin{tabular}{|c|c|c|c|c|c|c|c|}
\hline \multirow{2}{*}{ size } & \multicolumn{3}{|c|}{$\begin{array}{c}\Delta E \text { relative to GA_AL } \\
\text { (meV/atom) }\end{array}$} & \multicolumn{3}{|c|}{ Similarity Score } & \multirow{2}{*}{$\begin{array}{c}\text { Origin of } \\
\text { lowest-energy cluster }\end{array}$} \\
\hline & GA_AL & GA_DFT & Lit. & $\begin{array}{l}\text { GA_AL- } \\
\text { GA DFT }\end{array}$ & $\begin{array}{l}\text { GA_AL- } \\
\text { Lit. }\end{array}$ & $\begin{array}{c}\text { GA_DFT } \\
\text {-Lit. }\end{array}$ & \\
\hline 21 & 0.00 & 0.11 & 0.14 & 0.010 & 0.008 & 0.015 & GA_AL / GA_DFT / Lit. \\
\hline $22 *$ & 0.00 & -0.08 & 0.15 & 0.556 & 0.013 & 0.554 & GA_AL / GA_DFT / Lit. \\
\hline 23 & 0.00 & 0.06 & 1.31 & 0.009 & 0.766 & 0.767 & $\overline{\text { GA }}$ AL / GA DFT \\
\hline 24 & 0.00 & 0.03 & 9.98 & 0.009 & 1.611 & 1.614 & GA_AL / GA_DFT \\
\hline 25 & 0.00 & 2.03 & 10.11 & 0.560 & 1.672 & 1.460 & GA_AL \\
\hline 26 & 0.00 & 0.02 & 13.10 & 0.009 & 1.411 & 1.411 & GA_AL / GA_DFT \\
\hline 27 & 0.00 & 4.61 & 0.02 & 1.191 & 0.010 & 1.193 & GA AL / Lit. \\
\hline 28 & 0.00 & 0.00 & 0.04 & 0.018 & 0.019 & 0.006 & GA_AL / GA_DFT / Lit. \\
\hline 29 & 0.00 & -3.62 & 1.63 & 1.118 & 1.083 & 1.373 & GA_DFT \\
\hline 30 & 0.00 & 2.08 & 2.21 & 0.820 & 0.754 & 1.070 & GA AL \\
\hline 31 & 0.00 & 10.10 & -0.07 & 0.663 & 0.011 & 0.662 & GA_AL / Lit. \\
\hline 32 & 0.00 & 4.13 & -3.78 & 0.694 & 1.111 & 0.992 & Lit. \\
\hline $33 *$ & 0.00 & 1.46 & 0.06 & 0.240 & 0.008 & 0.241 & GA_AL / GA_DFT / Lit. \\
\hline 34 & 0.00 & 9.73 & 0.03 & 0.600 & 0.010 & 0.603 & GA AL / Lit. \\
\hline 35 & 0.00 & 0.08 & 40.99 & 0.008 & 0.953 & 0.953 & GA_AL / GA_DFT \\
\hline 36 & 0.00 & 0.02 & 51.81 & 0.008 & 1.090 & 1.089 & GA AL / GA DFT \\
\hline 37 & 0.00 & 5.26 & 40.87 & 0.611 & 1.525 & 1.337 & GA_AL \\
\hline 38 & 0.00 & 8.10 & 48.28 & 0.661 & 1.445 & 1.355 & GA $\mathrm{AL}$ \\
\hline 39 & 0.00 & 2.28 & 44.85 & 0.879 & 1.296 & 1.229 & GA_AL \\
\hline 40 & 0.00 & 2.29 & 32.45 & 1.022 & 1.011 & 1.213 & GA_AL \\
\hline 41 & 0.00 & 7.65 & 28.09 & 0.941 & 1.159 & 0.993 & $\mathrm{GA} \mathrm{AL}$ \\
\hline 42 & 0.00 & 10.55 & 31.44 & 0.609 & 0.986 & 1.086 & GA AL \\
\hline 43 & 0.00 & 24.24 & 35.38 & 1.138 & 1.100 & 1.047 & GA_AL \\
\hline 44 & 0.00 & 14.92 & 22.25 & 0.901 & 1.257 & 1.293 & GA_AL \\
\hline 45 & 0.00 & -1.37 & -1.38 & 1.600 & 1.602 & 0.027 & GA DFT / Lit. \\
\hline $46^{*}$ & 0.00 & 0.02 & 4.65 & 0.017 & 0.269 & 0.272 & GA AL / GA DFT \\
\hline 47 & 0.00 & 2.50 & 5.80 & 1.423 & 1.465 & 0.350 & GA AL \\
\hline 48 & 0.00 & 9.13 & 15.21 & 1.025 & 1.052 & 0.453 & GA AL \\
\hline 49 & 0.00 & 0.01 & 15.21 & 0.026 & 0.603 & 0.602 & GA_AL / GA_DFT \\
\hline 50 & 0.00 & -0.03 & 23.21 & 0.010 & 0.815 & 0.816 & GA AL / GA DFT \\
\hline 51 & 0.00 & 11.23 & 28.12 & 0.909 & 1.117 & 1.100 & GA_AL \\
\hline 52 & 0.00 & 13.91 & 33.77 & 1.118 & 1.462 & 1.238 & GA_AL \\
\hline 53 & 0.00 & 2.95 & 27.97 & 1.021 & 1.254 & 0.991 & GA_AL \\
\hline 54 & 0.00 & -4.00 & 28.11 & 1.009 & 1.117 & 1.383 & GA DFT \\
\hline 55 & 0.00 & 13.43 & -3.71 & 0.853 & 1.084 & 1.307 & Lit. \\
\hline
\end{tabular}




\section{Analysis of lowest-energy clusters with 21 to 55 atoms}

\subsection{Visualization of lowest-energy clusters}

Visualizations of the lowest-energy aluminum clusters with 21-55 atoms were generated using VESTA $^{29}$ and assembled into a panel in Figure S15. Symmetries were determined using Materials Studio $^{30}$ with a spatial tolerance of $0.1 \AA$. The lowest-energy cluster with 21 atoms has a dumbbell shape, which packs a 13-atom icosahedron at one end and a 14-atom variant resembling an icosahedron on the other end. From size 22 to 28 , the lowest-energy clusters gradually transform from irregular shapes to layered close-packed structures, with 27 and 28 having ordered layers in triangles stacked in the FCC stacking order. Lowest-energy configurations then start to alter the stacking order, with the cluster of 32 atoms forming a structure with closed-packed triangular layers stacking in HCP order. Starting from size 33, the lowest-energy isomers begin to favor tetrahedral shapes, and the 36-atom cluster has the most ordered shape and highest order of symmetry $\left(\mathrm{D}_{2 \mathrm{~d}}\right)$ among all lowest-energy clusters. After 36, the preference of lowest-energy morphology changes back to layered close-packed structure. Lowest-energy clusters with 37, 38 and 39 atoms form transitional shapes between tetrahedron and layered configuration. Lowestenergy clusters with 40 to 43 atoms regain the FCC stacking order with close-packed layers, some of which have trapezoidal shapes instead of triangular. The 44-atom lowest-energy isomer loses the layered morphology but mostly retrains close-packed outer surfaces. Lowest-energy clusters of 45 and 46 have spherical shapes, while the cluster with 47 atoms have a layered structure with HCP stacking order. From size 49 to 53, lowest-energy clusters are neither layered nor tetrahedral, but still favor close-packed outer surfaces. Finally, the 54-atom and 55-atom lowest-energy structure transform back to tetrahedral configurations with close-packed surfaces. The preferred morphologies adopted by lowest-energy aluminum clusters periodically transform between layered close-packed structures and tetrahedrons with close-packed outer surfaces. This observation reflects the facts that $\mathrm{Al}$ in its bulk phase has an FCC crystal structure, and (111) surfaces possess the lowest free surface energies among low-index surfaces. ${ }^{31}$ The prevalence of preferred morphologies for lowest-energy isomers across a wide range of sizes also suggests that the seeding operation, which generates new clusters from seed structures of other sizes, can potentially accelerate ground state search by a large extent, when low-energy structures of neighboring sizes are available. 


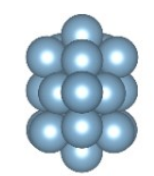

21

$\left(\mathrm{C}_{\mathrm{s}}\right)$

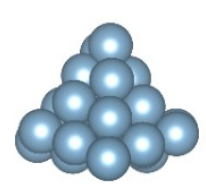

26

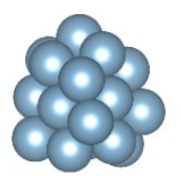

22_01

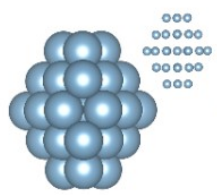

27

$\left(\mathrm{C}_{2 \mathrm{v}}\right)$

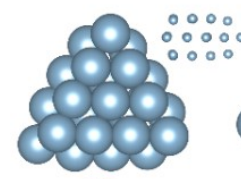

32

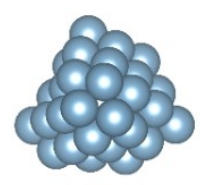

38

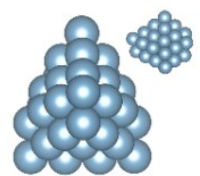

44

$\left(\mathrm{C}_{\mathrm{s}}\right)$

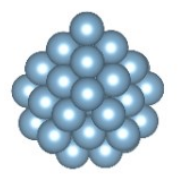

50

$\left(\mathrm{C}_{2}\right)$

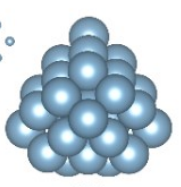

33

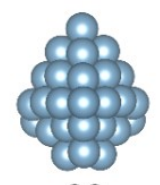

39

$\left(\mathrm{C}_{\mathrm{s}}\right)$
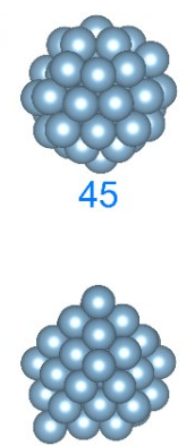

51

$\left(C_{s}\right)$

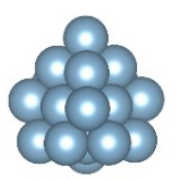

22_02

$\left(\mathrm{C}_{\mathrm{s}}\right)$

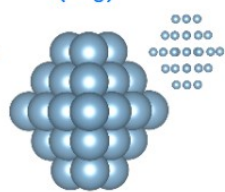

28

$\left(\mathrm{C}_{2 \mathrm{v}}\right)$

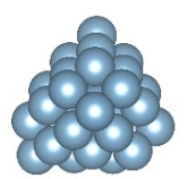

34

$\left(\mathrm{C}_{2}\right)$

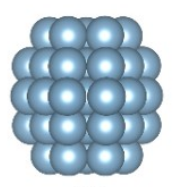

40

$\left(\mathrm{C}_{2 \mathrm{v}}\right)$

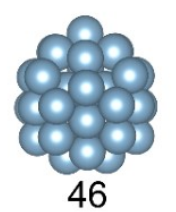

$\left(\mathrm{C}_{\mathrm{s}}\right)$

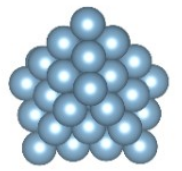

52

$\left(\mathrm{S}_{4}\right)$

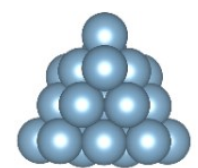

23

$\left(\mathrm{C}_{3 \mathrm{v}}\right)$

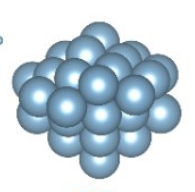

29

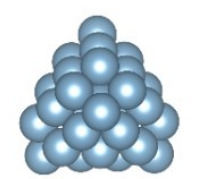

35

$\left(\mathrm{C}_{\mathrm{s}}\right)$

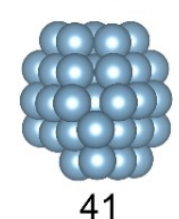

41

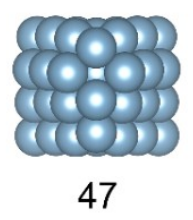

$\left(\mathrm{C}_{\mathrm{s}}\right)$

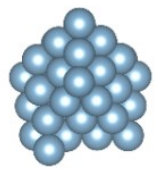

53

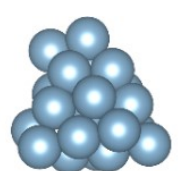

24

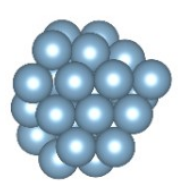

30

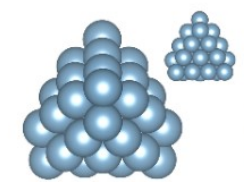

36

$\left(D_{2 d}\right)$

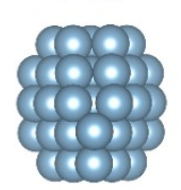

42

(C)

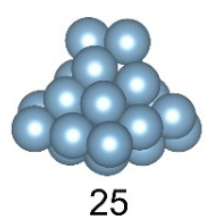

$\left(\mathrm{C}_{2}\right)$
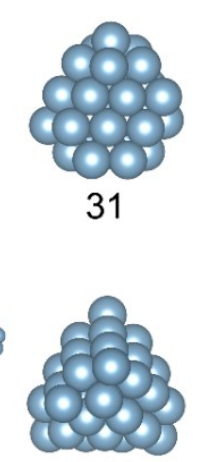

37

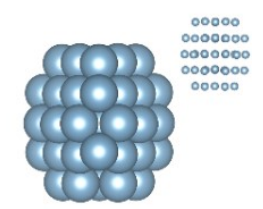

43

$\left(\mathrm{C}_{\mathrm{s}}\right)$
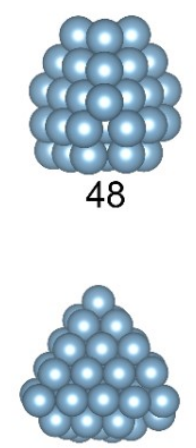

54

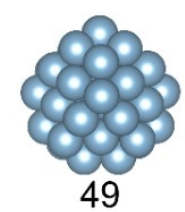

Figure S15. Lowest-energy clusters assembled from GA_AL, GA_DFT and the literature. Labels of the lowest-lowest energy clusters discovered by GA_AL and GA_DFT are colored in black and blue, while the ones reported in the literature ${ }^{19, \overline{27}}$ are labelled in red. Proposed symmetries are included below corresponding clusters. Informative alternative views are put at the top right corner.

\subsection{Stability Analysis}

To measure relative stabilities, cohesive energies, binding energies, and the second energy differences were calculated and plotted in Figure S16. The cohesive energy is defined as the energy required to break the cluster into isolated neutral atoms at $0 \mathrm{~K}$ and is computed by

$$
E_{c}(n)=\left[n \times E_{1}-E_{n}\right] / n,
$$


where $E_{c}(n), E_{1}$, and $E_{n}$ represent the cohesive energy per atom for an n-atom cluster, the total energy of an isolated aluminum atom, and the total energy of a cluster with $n$ atoms respectively. The binding energy measures the energy necessary to break one atom from the cluster and is calculated as

$$
E_{b}(n)=E_{n-1}+E_{1}-E_{n}
$$

where $E_{b}(n)$ represents the binding energy and the other symbols carry the same meaning as those in equation (5). The second energy difference describes the stability of the lowest-energy cluster of one size relative to the adjacent sizes and is calculated as

$$
\Delta_{2} E(n)=E_{n+1}+E_{n-1}-2 E_{n}
$$

of which a positive value implies higher stability.

Cohesive energies steadily increase as clusters grow, and values of all sizes are still well below the bulk value of $3.39 \mathrm{eV} / \mathrm{atom},{ }^{32}$ suggesting clusters in this size are significantly less stable than the bulk crystal. The binding energies and the second energy differences display an odd-even oscillation, which can be attributed to the existence of one unpaired electron in odd-sized clusters. The lowest-energy cluster with 36 atoms exhibits peaks in both the second energy differences and cohesive energies, meaning it is more stable than lowest-energy clusters of neighboring sizes. This suggests it could exist in relatively high abundance in gas-phase experiments and agrees with the experimental observation of surprisingly high melting temperatures of Al cation clusters with around 36 atoms. ${ }^{33,34}$

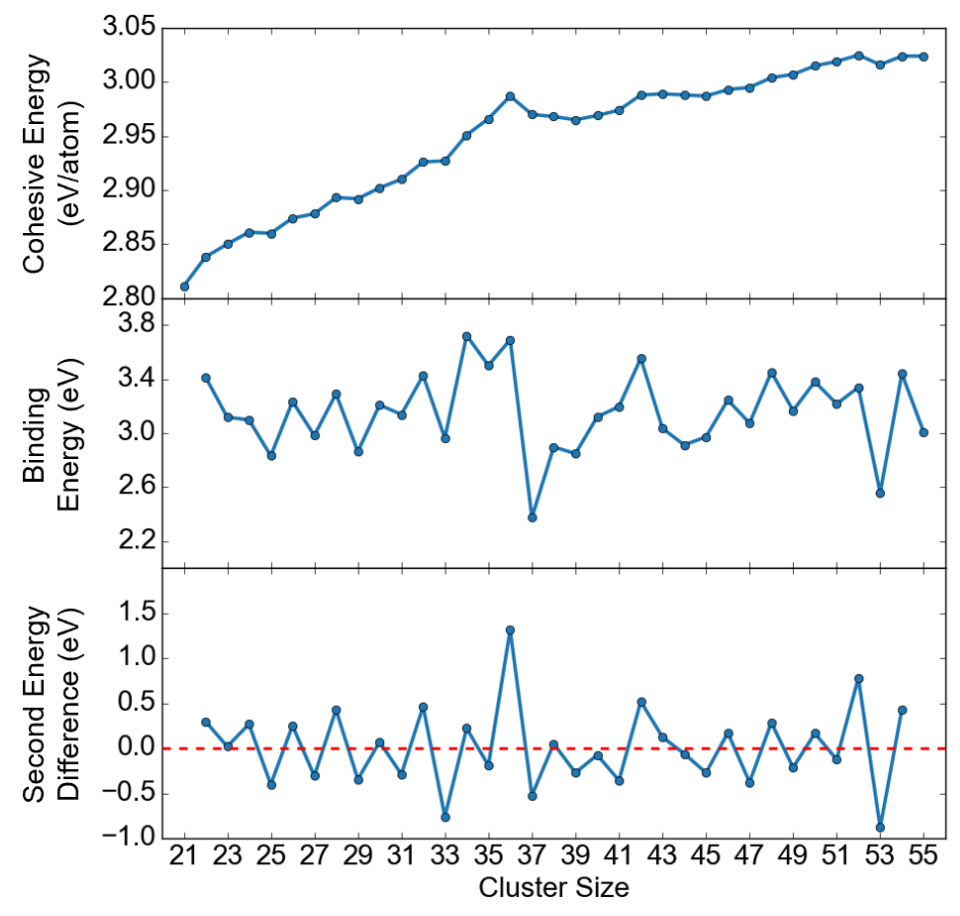

Figure S16. Cohesive energy, binding energy and second energy difference for clusters with 21 to 55 atoms. 


\section{Relationship between extrapolation grade and energy prediction error}

Although high extrapolation grades generally indicate a large predication error, lower extrapolation grades below the extrapolation threshold (1.0) do not necessarily guarantee smaller prediction errors. Figure S17 shows the extrapolation grades of DFT-relaxed clusters at each retraining step for GA_AL runs on clusters with 21, 38 and 55 atoms. We focus on the local ground states of DFT relaxations because we are primarily interested in low-energy structures for the purpose of the structure search. Extrapolation grades decrease as more retraining steps were performed. However, the prediction errors of those having extrapolation grades smaller than 1.0 scatter randomly with a tendency of over-estimating the total energy (i.e., MTP predicted an energy higher than corresponding DFT energy).

a)

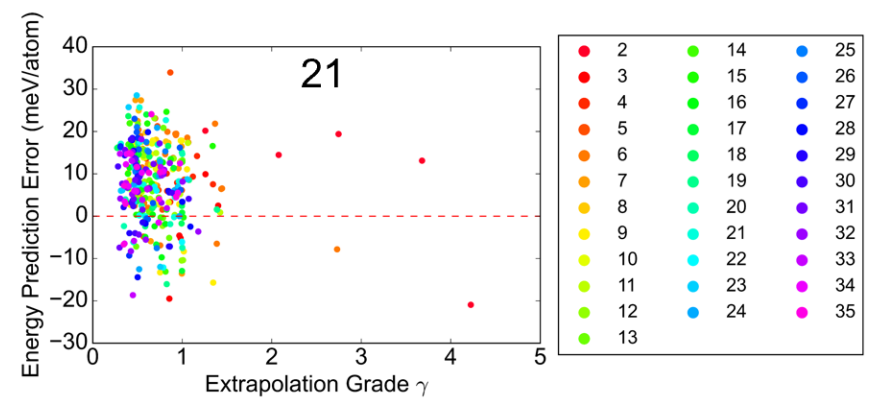

b)

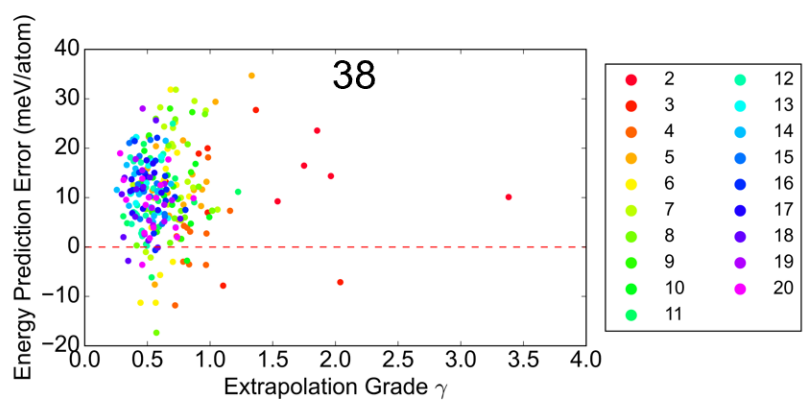

c)

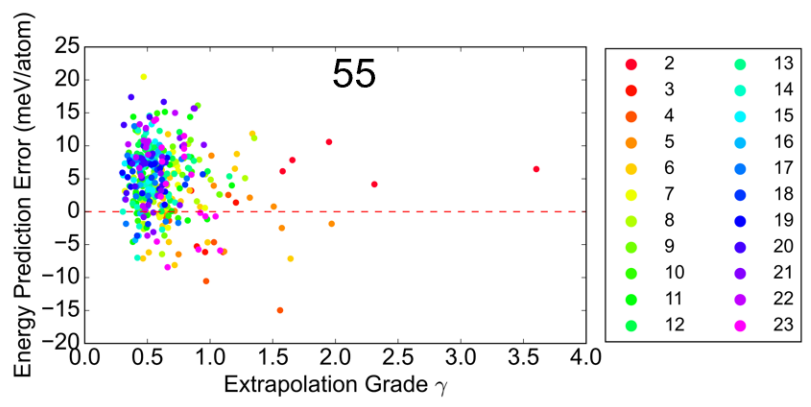

Figure S17. Energy prediction errors against extrapolation grades for ground states of DFT calculations at retraining steps of GA_AL for clusters with a) 21, b) 38 and c) 55 atoms. The legends represent the retraining step of a GA_AL run. 


\section{References}

1 Holland, J. H. Adaptation in natural and artificial systems. (University of Michigan Press, 1975).

2 Ho, K.-M. et al. Structures of medium-sized silicon clusters. Nature 392, 582-585, doi:10.1038/33369 (1998).

3 Johnston, R. L. Evolving better nanoparticles: Genetic algorithms for optimising cluster geometries. Dalton Transactions, 4193-4207, doi:10.1039/B305686D (2003).

4 Vilhelmsen, L. B. \& Hammer, B. A genetic algorithm for first principles global structure optimization of supported nano structures. J Chem Phys 141 (2014).

5 Shayeghi, A., Götz, D., Davis, J. B. A., Schäfer, R. \& Johnston, R. L. Pool-BCGA: a parallelised generation-free genetic algorithm for the ab initio global optimisation of nanoalloy clusters. Phys Chem Chem Phys 17, 2104-2112, doi:10.1039/C4CP04323E (2015).

6 Aslan, M., Davis, J. B. A. \& Johnston, R. L. Global optimization of small bimetallic PdCo binary nanoalloy clusters: a genetic algorithm approach at the DFT level. Physical Chemistry Chemical Physics 18, 6676-6682, doi:10.1039/C6CP00342G (2016).

7 Sun, J., Xie, X., Cao, B. \& Duan, H. A density-functional theory study of $\mathrm{Au}_{13}, \mathrm{Pt}_{13}, \mathrm{Au}_{12} \mathrm{Pt}$ and $\mathrm{Pt}_{12} \mathrm{Au}$ clusters. Computational and Theoretical Chemistry 1107, 127-135 (2017).

8 Buendía, F., Vargas, J. A., Johnston, R. L. \& Beltrán, M. R. Study of the stability of small AuRh clusters found by a Genetic Algorithm methodology. Computational and Theoretical Chemistry 1119, 51-58, doi:https://doi.org/10.1016/j.comptc.2017.09.008 (2017).

9 Tipton, W. W., Bealing, C. R., Mathew, K. \& Hennig, R. G. Structures, phase stabilities, and electrical potentials of Li-Si battery anode materials. Physical Review B 87, 184114, doi:10.1103/PhysRevB.87.184114 (2013).

10 Tipton, W. W. \& Hennig, R. G. A grand canonical genetic algorithm for the prediction of multi-component phase diagrams and testing of empirical potentials. Journal of Physics: Condensed Matter 25, 495401, doi:10.1088/0953-8984/25/49/495401 (2013).

11 Dong, R., Chen, X., Wang, X. \& Lu, W. Structural transition of hexagonal tube to rocksalt for $(\mathrm{MgO})_{3 \mathrm{n}}, 2 \leqslant \mathrm{n} \leqslant 10$. The Journal of Chemical Physics 129, 044705, doi:10.1063/1.2956508 (2008).

12 Choi, I.-G. et al. Energetically-favorable distribution of oxygen vacancies and metal atoms in perovskite $\mathrm{BaCe}_{\mathrm{x}} \mathrm{Zr}_{0.85-\mathrm{x}} \mathrm{Y}_{0.15} \mathrm{O}_{2.925}$ solid solutions using a genetic algorithm and lattice $\begin{array}{lllll}\text { statics. } & \text { Comp } & \text { Mater } & \text { 170, }\end{array}$ doi:https://doi.org/10.1016/j.commatsci.2019.109184 (2019).

13 Collins, S. P., Daff, T. D., Piotrkowski, S. S. \& Woo, T. K. Materials design by evolutionary optimization of functional groups in metal-organic frameworks. Science Advances 2, e1600954, doi:10.1126/sciadv.1600954 (2016).

14 Deaven, D. M. \& Ho, K. M. Molecular-Geometry Optimization with a Genetic Algorithm. Phys Rev Lett 75, 288-291 (1995).

15 Kresse, G. \& Furthmuller, J. Efficiency of ab-initio total energy calculations for metals and semiconductors using a plane-wave basis set. Comp Mater Sci 6, 15-50 (1996).

16 Kresse, G. \& Furthmuller, J. Efficient iterative schemes for ab initio total-energy calculations using a plane-wave basis set. Physical Review B 54, 11169-11186 (1996).

17 Kresse, G. \& Hafner, J. Abinitio Molecular-Dynamics for Liquid-Metals. Physical Review $B$ 47, 558-561 (1993). 
18 Plimpton, S. Fast Parallel Algorithms for Short-Range Molecular Dynamics. J Comput Phys 117, 1-19, doi:https://doi.org/10.1006/jeph.1995.1039 (1995).

19 Piotrowski, M. J. et al. Theoretical Study of the Structural, Energetic, and Electronic Properties of 55-Atom Metal Nanoclusters: A DFT Investigation within van der Waals Corrections, Spin-Orbit Coupling, and $\mathrm{PBE}+\mathrm{U}$ of 42 Metal Systems. The Journal of Physical Chemistry C 120, 28844-28856, doi:10.1021/acs.jpcc.6b10404 (2016).

20 Novikov, I. S., Gubaev, K., Podryabinkin, E. V. \& Shapeev, A. V. The MLIP package: moment tensor potentials with MPI and active learning. Machine Learning: Science and Technology 2, 025002, doi:10.1088/2632-2153/abc9fe (2021).

21 Broyden, C. G. The Convergence of a Class of Double-rank Minimization Algorithms 1. General Considerations. IMA Journal of Applied Mathematics 6, 76-90, doi:10.1093/imamat/6.1.76 (1970).

22 Fletcher, R. A new approach to variable metric algorithms. The Computer Journal 13, 317 322, doi:10.1093/comjnl/13.3.317 (1970).

23 Goldfarb, D. A family of variable-metric methods derived by variational means. Mathematics of computation 24, 23-26 (1970).

24 Shanno, D. F. Conditioning of quasi-Newton methods for function minimization. Mathematics of computation 24, 647-656 (1970).

25 Manna, S. et al. A Database to Enable the Design and Discovery of Atomically Precise Nanoclusters. (Manuscript in preparation).

26 Doye, J. P. K. A model metal potential exhibiting polytetrahedral clusters. The Journal of Chemical Physics 119, 1136-1147, doi:10.1063/1.1574797 (2003).

27 Aguado, A. \& López, J. M. Structures and stabilities of $\mathrm{Al}_{\mathrm{n}}^{+}, \mathrm{Al}_{\mathrm{n}}$, and $\mathrm{Al}_{\mathrm{n}}{ }^{-}(\mathrm{n}=13-34)$ clusters. The Journal of Chemical Physics 130, 064704, doi:10.1063/1.3075834 (2009).

28 Tuo, P., Ye, X. B. \& Pan, B. C. A machine learning based deep potential for seeking the low-lying candidates of Al clusters. The Journal of Chemical Physics 152, 114105, doi:10.1063/5.0001491 (2020).

29 Momma, K. \& Izumi, F. VESTA 3 for three-dimensional visualization of crystal, volumetric and morphology data. Journal of Applied Crystallography 44, 1272-1276, doi:10.1107/s0021889811038970 (2011).

30 BIOVIA, Dassault Systèmes, Materials Studio (San Diego: Dassault Systèmes).

31 Patra, A., Bates, J. E., Sun, J. \& Perdew, J. P. Properties of real metallic surfaces: Effects of density functional semilocality and van der Waals nonlocality. Proceedings of the National Academy of Sciences 114, E9188, doi:10.1073/pnas. 1713320114 (2017).

32 Kittel, C. Introduction to solid state physics. (Wiley, 2005).

33 Neal, C. M., Starace, A. K. \& Jarrold, M. F. Melting transitions in aluminum clusters: The role of partially melted intermediates. Physical Review B 76, 054113, doi:10.1103/PhysRevB.76.054113 (2007).

34 Aguado, A. \& Jarrold, M. F. Melting and Freezing of Metal Clusters. Annual Review of Physical Chemistry 62, 151-172, doi:10.1146/annurev-physchem-032210-103454 (2011). 


\section{Supplementary File: Atomic Coordinates for Clusters}

\section{for \\ "Accelerated Prediction of Atomically Precise Cluster Structures Using On-the-fly Machine Learning"}

\section{Table of Contents}

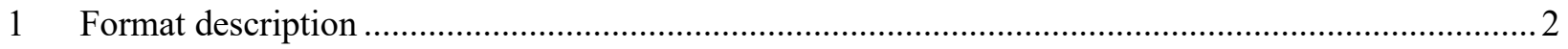

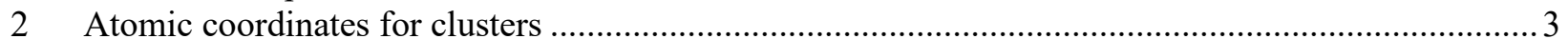

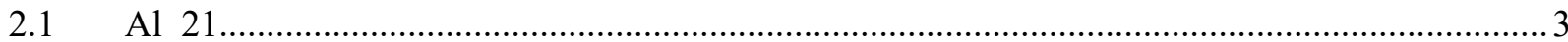

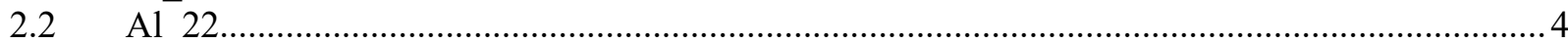

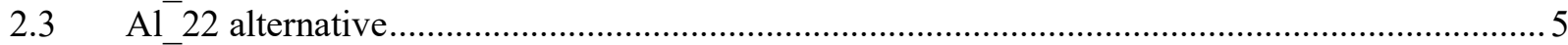

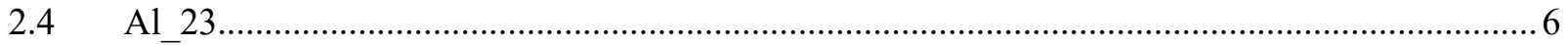

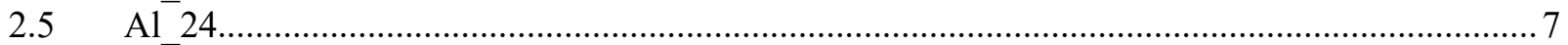

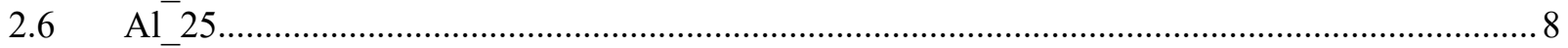

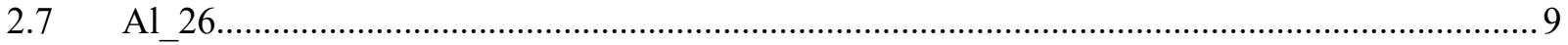

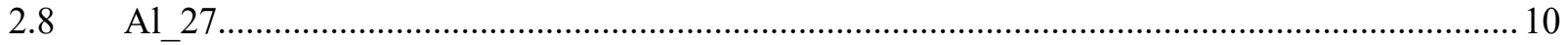

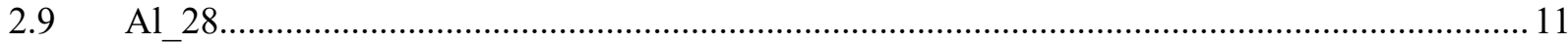

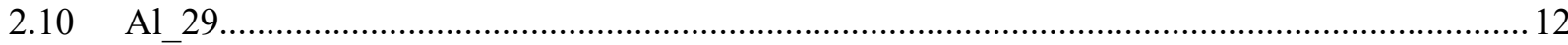

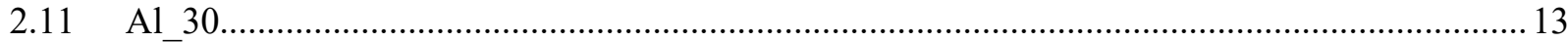

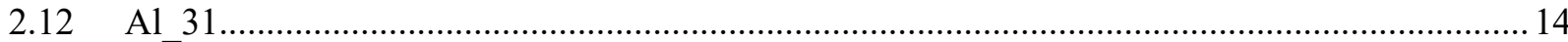

2.13 Al_32

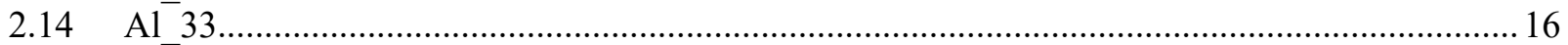

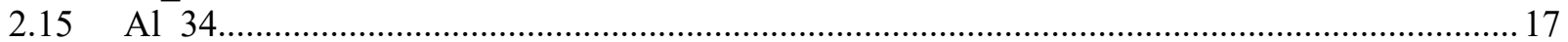

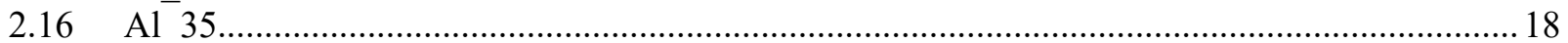

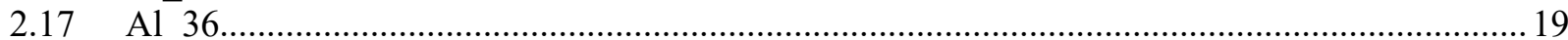

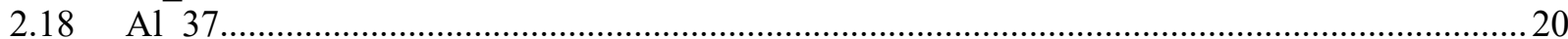

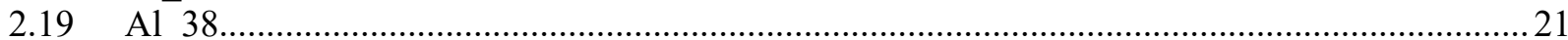

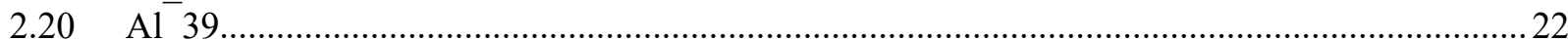

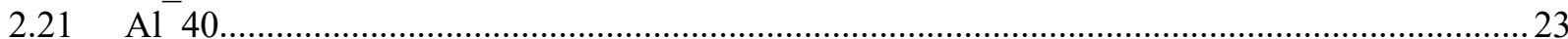

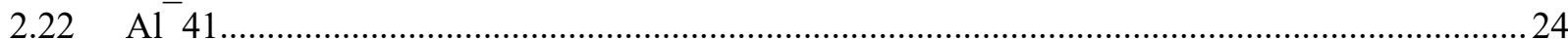

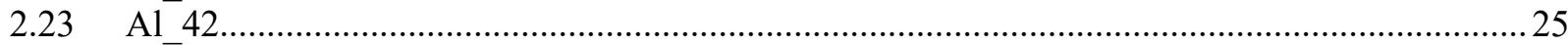

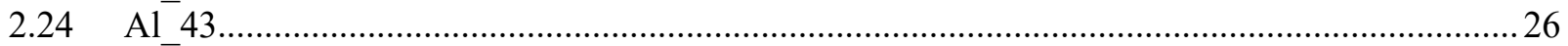

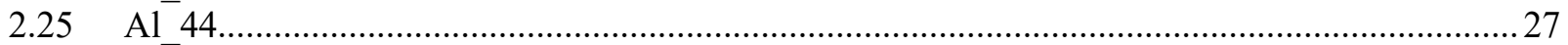

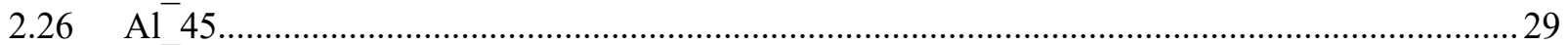

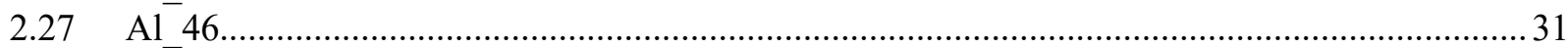

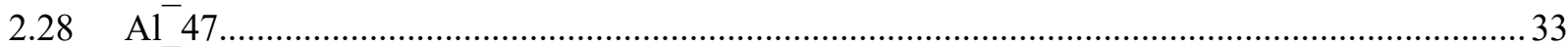

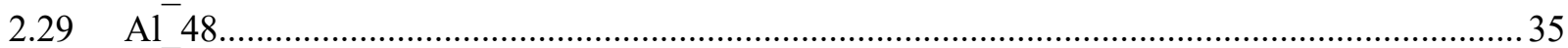

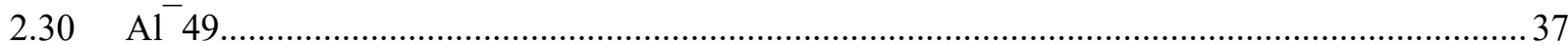

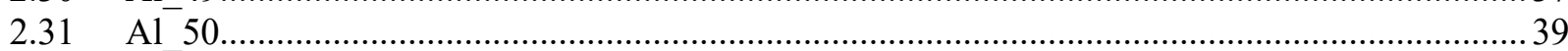

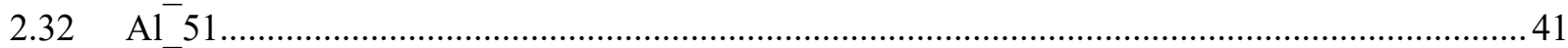

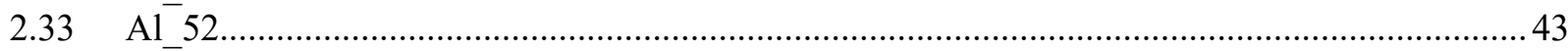

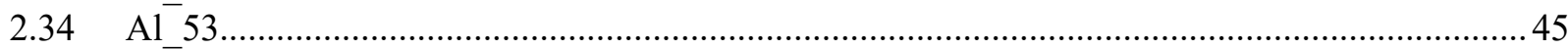

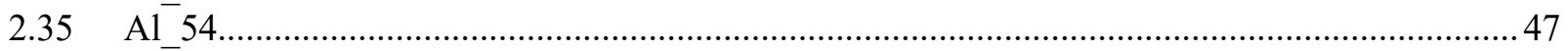

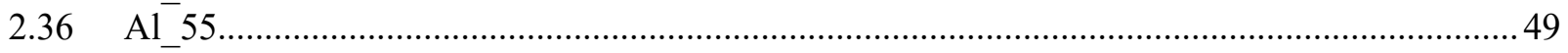




\section{Format description}

The following sections list the atomic coordinates of the lowest-energy structures discovered in this work and reported in literature for aluminum nanoclusters with 21 to 55 atoms. For clusters with 22 atoms, a cluster which has total energies close to the lowest-energy structure (difference below $0.1 \mathrm{meV} /$ atom) but is structurally dissimilar is also listed.

Clusters are provided in the XYZ format with the center of mass positioned at the origin. The first line provides the number of atoms of the cluster, with a trailing blank comment line. The following lines list the element name and atomic coordinates in the Cartesian coordinate system. 


\section{Atomic coordinates for clusters}

$\begin{array}{lrrr}\mathbf{2 . 1} & \text { Al_21 } & & \\ 21 & & & \\ & & & \\ \text { Al } & -0.596503690031571 & 0.159271003097976 & -3.637482070185255 \\ \text { Al } & 2.286585294345931 & 0.830951886913486 & -2.958567160431452 \\ \text { Al } & 1.033664713140647 & -1.711617999236393 & -2.828193465536144 \\ \text { Al } & -0.042429815500894 & 2.514381907574577 & -2.676371340719141 \\ \text { Al } & -1.994125385900171 & -0.685373895053791 & -1.472730847744709 \\ \text { Al } & 0.612813037104070 & 0.428119171309620 & -1.022610715335517 \\ \text { Al } & 1.833938631530993 & 3.051752633436998 & -0.952245932007926 \\ \text { Al } & 3.291204481516125 & 0.981503395903907 & -0.306840864540883 \\ \text { Al } & 2.789670119756137 & -1.552478690758089 & -0.867246860234246 \\ \text { Al } & -1.664565852575888 & 2.027852564097188 & -0.560851053283410 \\ \text { Al } & 0.009875492165804 & -2.207470007291898 & -0.318426825750153 \\ \text { Al } & -3.604814527091738 & 0.276220671025232 & 0.598594008319584 \\ \text { Al } & -2.522434942937107 & -2.342980190580137 & 0.716628552719046 \\ \text { Al } & -0.985441439688779 & -0.079146205215778 & 0.984734309665225 \\ \text { Al } & 0.542902588090966 & 2.164276391641030 & 1.265649863144239 \\ \text { Al } & 1.644517023945994 & -0.349686734470989 & 1.467023095161313 \\ \text { Al } & 2.126418896541706 & -3.156685964118831 & 1.350095917319861 \\ \text { Al } & -2.058244515402470 & 2.002878305992782 & 2.268744418261399 \\ \text { Al } & -0.197081901030137 & -2.178984135548673 & 2.442399216653241 \\ \text { Al } & -2.523049637430812 & -0.694121355932231 & 3.032404506840242 \\ \text { Al } & 0.017101429451174 & 0.521337247214026 & 3.475293247684695\end{array}$




\subsection{Al_22}

22

$\begin{array}{lrrr}\text { Al } & 0.849728635095090 & 0.305009796490726 & -3.641055973590975 \\ \text { Al } & -0.454824354095036 & 2.443823170741856 & -2.806899464901289 \\ \text { Al } & 0.200621843188900 & -2.141129322536001 & -2.552127948594587 \\ \text { Al } & 2.782544148441950 & -0.774659877732478 & -2.197050280925546 \\ \text { Al } & -2.394278740440318 & -2.130111060577140 & -1.676982107194558 \\ \text { Al } & -0.854519914911067 & 0.085643404371087 & -1.434072955075608 \\ \text { Al } & -2.644206095113693 & 2.085007797081076 & -1.389093002230260 \\ \text { Al } & 1.625569506058016 & 1.598954010301494 & -1.345107336727165 \\ \text { Al } & -3.752206017392702 & -0.164218898227926 & -0.568191139334245 \\ \text { Al } & -0.689465884416474 & -3.752523983064122 & -0.344462829080266 \\ \text { Al } & 1.259316911884746 & -1.841946607729521 & -0.183061918330887 \\ \text { Al } & -0.411116837144375 & 2.845396321557978 & -0.042262882594020 \\ \text { Al } & 3.220950536848502 & 0.151081614879093 & 0.366981695883849 \\ \text { Al } & -1.627935327892790 & -1.409536831307928 & 0.753515558904583 \\ \text { Al } & 0.461794221361892 & 0.421670607211276 & 0.952522431393229 \\ \text { Al } & 2.077040383287498 & 2.576923997608393 & 1.110497092794137 \\ \text { Al } & -2.366524639077721 & 1.077835212236092 & 1.359454454187127 \\ \text { Al } & 0.093044330099149 & -2.968377169100195 & 2.070980544702035 \\ \text { Al } & -0.467048104567413 & 2.399369999028631 & 2.677518521706466 \\ \text { Al } & 2.144295536701726 & -1.318854489262010 & 2.370380580588957 \\ \text { Al } & -0.747427286109438 & -0.477667969318798 & 3.149598326185417 \\ \text { Al } & 1.694647148193528 & 0.988310277348424 & 3.368918632233607\end{array}$




\subsection{Al 22 alternative}

22

$\begin{array}{lccc}\text { Al } & -0.273801078607146 & -1.340367421326714 & -3.539314417717854 \\ \text { Al } & 0.631529234117727 & 1.279665740298393 & -3.239023148413977 \\ \text { Al } & -2.444039637864109 & -1.927089695781956 & -2.177553151957000 \\ \text { Al } & 1.828964825895078 & -0.828965378053382 & -2.064753233220965 \\ \text { Al } & -1.297437102997620 & 0.356793147320385 & -1.596541602866242 \\ \text { Al } & 2.543496086157049 & 1.773103591436458 & -1.359611348464474 \\ \text { Al } & -0.143376031862703 & 2.799614407693678 & -1.124717451371499 \\ \text { Al } & -3.680389476081229 & -0.199129038131561 & -0.414782086796510 \\ \text { Al } & -0.180165326010709 & -2.204536632151846 & -0.636085018634361 \\ \text { Al } & 2.487504027315685 & -2.792688091329786 & -0.529716013261412 \\ \text { Al } & -2.333658271352760 & 1.995225721572432 & 0.329205761692021 \\ \text { Al } & 0.691084087782505 & 0.415975152092672 & 0.177439435331653 \\ \text { Al } & 3.365751811688295 & -0.344826198924196 & 0.239165595951697 \\ \text { Al } & -2.690379192424030 & -2.644302807473455 & 0.389749848834397 \\ \text { Al } & -0.784357279261744 & 4.114047510257121 & 1.071995641485788 \\ \text { Al } & 1.602456637337488 & 2.883314037886288 & 0.954819139639666 \\ \text { Al } & 2.154889262873093 & 0.695381808328641 & 2.507738222190516 \\ \text { Al } & 1.454132773891089 & -1.857863952216194 & 1.639150680508083 \\ \text { Al } & -1.572787833253606 & -0.449174592458125 & 1.286605771852454 \\ \text { Al } & -0.847037290909182 & -2.953433510123781 & 2.237982687877929 \\ \text { Al } & -0.476532277352966 & 1.827788708617755 & 2.351101666908820 \\ \text { Al } & -0.035847949080184 & -0.598532507532829 & 3.497143020431277\end{array}$




\subsection{Al 23}

23

$\begin{array}{lrrr}\text { Al } & -1.056193001906759 & 1.777458535036345 & -4.036878242070214 \\ \text { Al } & -1.662845417163185 & -0.872606028202219 & -3.488247009877295 \\ \text { Al } & 0.653104718818746 & 0.286429079172084 & -2.656690502518004 \\ \text { Al } & 0.089152507202098 & -2.255358054416752 & -1.927324851136791 \\ \text { Al } & 0.156320098678565 & 2.904917665603040 & -1.876940119742804 \\ \text { Al } & -1.932600585443816 & 1.091473573747804 & -1.626174040440808 \\ \text { Al } & 2.464785313255726 & 1.716796485701495 & -1.298765167302959 \\ \text { Al } & 2.318705617130146 & -0.951961493967749 & -1.129356311861211 \\ \text { Al } & -2.395356756729070 & -1.481344920235703 & -0.936044132261309 \\ \text { Al } & -0.589089941602092 & -2.806354368148527 & 0.714849590896245 \\ \text { Al } & 1.856699371701371 & -3.483994907661041 & -0.259740100527672 \\ \text { Al } & 0.191299236238606 & 0.430005959652693 & 0.145207098402887 \\ \text { Al } & -1.433579596687037 & 2.930858856737441 & 0.255628372498501 \\ \text { Al } & 3.275887497917266 & 0.592648304426140 & 0.971365198075032 \\ \text { Al } & 1.279634692163729 & 2.904809352806383 & 0.939964029986895 \\ \text { Al } & -2.562934555885653 & 0.568457707329271 & 0.817290499861969 \\ \text { Al } & 1.615349035816470 & -1.534377231181534 & 1.625564926792130 \\ \text { Al } & -2.992212418616184 & -1.973084496731546 & 1.674942847302498 \\ \text { Al } & 4.027255095758347 & -1.997848032972129 & 0.613465689831806 \\ \text { Al } & -0.684719772516469 & -0.817934721112872 & 2.544071910146068 \\ \text { Al } & -2.985833973130648 & 0.187957288239364 & 3.410903288896693 \\ \text { Al } & 1.395881851760645 & 0.849361539831863 & 2.835401906179976 \\ \text { Al } & -1.028709016760793 & 1.933689906346157 & 2.687505118868362\end{array}$




\subsection{Al_24}

24

$\begin{array}{lrrr}\text { Al } & -0.433675090612311 & -3.258981745336208 & -3.378502173492810 \\ \text { Al } & 0.975993444053721 & -1.141796800024916 & -2.714739942358611 \\ \text { Al } & -0.406944439062864 & 1.112899725983780 & -2.548662634934395 \\ \text { Al } & -1.795507514705186 & -1.229779723794793 & -2.355963455822907 \\ \text { Al } & 1.990309770384018 & -3.674831856940134 & -2.175416678832782 \\ \text { Al } & 1.154584705385499 & 3.257141858896498 & -1.762800392526183 \\ \text { Al } & 2.270405035823764 & 0.766150852993876 & -1.672058591354554 \\ \text { Al } & -2.746882840456522 & 0.781275028755681 & -1.059218370740322 \\ \text { Al } & -1.261365489727298 & 3.011745934298293 & -0.717479950215399 \\ \text { Al } & -0.298114585330627 & -2.991831670807974 & -0.768205229815478 \\ \text { Al } & 3.381195691765104 & 2.953934334762456 & -0.400716225806839 \\ \text { Al } & 2.085592426481522 & -1.670240935735511 & -0.210946215893742 \\ \text { Al } & -0.083385372808253 & 0.028157967689429 & -0.059502118294979 \\ \text { Al } & -2.254062459338492 & -1.600119730350939 & 0.305774694150244 \\ \text { Al } & -3.659666402918445 & 2.940422847274382 & 0.541119587657548 \\ \text { Al } & 0.968435432854637 & 2.632472455922597 & 0.860314543922312 \\ \text { Al } & 2.899900445572504 & 0.717613116960974 & 0.964069819991568 \\ \text { Al } & -3.662661194791258 & 0.445080110257445 & 1.469965630185792 \\ \text { Al } & -1.382412018774617 & 1.909540911259665 & 1.747158357600874 \\ \text { Al } & 0.113949285254231 & -2.363662275207487 & 1.655713522091370 \\ \text { Al } & -1.577724554743486 & -0.582082277879082 & 2.789060302438751 \\ \text { Al } & 0.781898802339503 & 0.773829264452365 & 2.691423146855286 \\ \text { Al } & 0.460320630913570 & -1.474161478643536 & 4.240447385427561 \\ \text { Al } & 2.479816292441303 & -1.342775914786874 & 2.559164989767694\end{array}$




$\begin{array}{llll}\mathbf{2 . 6} & \text { Al_25 } & & \\ \text { 25 } & & & \\ & & & \\ \text { Al } & 1.628563383619499 & -2.624387910024781 & -2.111071705976232 \\ \text { Al } & -0.821696822560490 & -1.446565006798433 & -2.336395021395763 \\ \text { Al } & 1.492074868923996 & 0.074386302774872 & -2.453532584112666 \\ \text { Al } & 3.826591781869309 & -1.227357935876961 & -2.637803243939576 \\ \text { Al } & -0.832161932449985 & 1.092825957232115 & -2.748175570065385 \\ \text { Al } & -2.690695100695137 & 0.105368653977994 & -0.921306739788555 \\ \text { Al } & -3.043567168900930 & 2.497247908695186 & -2.203179095981397 \\ \text { Al } & 3.840650452350468 & 1.032439502737924 & -1.237340847512977 \\ \text { Al } & 1.219726208090211 & 2.366301839941899 & -0.949605723206790 \\ \text { Al } & -0.472394767765564 & -4.116944395630505 & -1.606064581936121 \\ \text { Al } & -0.619433214951366 & 3.720962222388939 & -2.460165055431859 \\ \text { Al } & -1.421225692215415 & 2.715891969257697 & -0.116056125714143 \\ \text { Al } & -2.028719624739835 & -2.523330114241594 & -0.207692972251067 \\ \text { Al } & 2.705179925893926 & -1.111959412037868 & -0.087554927040285 \\ \text { Al } & -0.012458736166073 & -0.016658414538014 & -0.014930779166127 \\ \text { Al } & 0.669178464717639 & -2.732531845864647 & 0.504292626839518 \\ \text { Al } & 2.792092707439046 & 1.317997849142156 & 1.140935307668466 \\ \text { Al } & -3.643879173553937 & -1.364357759733855 & 1.489394563634551 \\ \text { Al } & 0.338576296641282 & 2.229903243699887 & 1.697248483423444 \\ \text { Al } & -2.124608813550182 & 0.989880163624363 & 1.630294576112416 \\ \text { Al } & 1.482257833396979 & -0.894685770593627 & 2.239271296685054 \\ \text { Al } & -1.066137956879192 & -1.659377157889812 & 2.186988800428161 \\ \text { Al } & 2.089700542531453 & 1.374332744449115 & 3.674168122345407 \\ \text { Al } & -0.405227625559105 & 0.546057464111200 & 3.700958047238545 \\ \text { Al } & -2.902385835486625 & -0.345440098803257 & 3.827323149143382\end{array}$




\subsection{Al_26 \\ 26}

$\begin{array}{lrrr}\text { Al } & 3.782683677001378 & -0.418392815828973 & -2.858410831689532 \\ \text { Al } & -0.486763736986296 & -1.521568889561746 & -2.132062776534922 \\ \text { Al } & -3.061260669639445 & -2.233174507576222 & -2.778958895199447 \\ \text { Al } & 1.278781780032805 & 0.386113413412104 & -2.724295337231490 \\ \text { Al } & -3.242467759016775 & 0.379278399713737 & -3.266240833033504 \\ \text { Al } & -0.942711248110358 & 1.422049810851082 & -2.086586893481969 \\ \text { Al } & 3.319608308251988 & 2.247295420261866 & -2.552728267623671 \\ \text { Al } & 2.130190478968743 & -1.950748659655162 & -1.403189018998420 \\ \text { Al } & -3.389396423456468 & 2.198437020405265 & -1.274109547490195 \\ \text { Al } & 3.126251146198289 & 0.463867834189687 & -0.470417668738532 \\ \text { Al } & -2.685925525552402 & -0.422228871048732 & -0.760175795720453 \\ \text { Al } & 1.223725954615784 & 2.458445439292221 & -0.710893364332282 \\ \text { Al } & -2.213486995810824 & -3.159129960584931 & -0.466013162577058 \\ \text { Al } & 0.193643523973424 & -0.039498752344796 & 0.064307418526331 \\ \text { Al } & -1.320413924249091 & 3.496414485451142 & -0.310319825099564 \\ \text { Al } & 0.361861763515163 & -2.884885236303719 & 0.253447002852095 \\ \text { Al } & 2.321575244991342 & -1.324110547363793 & 1.289829694318291 \\ \text { Al } & -2.139019341650411 & 1.338761436057421 & 1.123230054181722 \\ \text { Al } & 2.048569723587674 & 1.382816798016947 & 1.698110458513286 \\ \text { Al } & -1.868982742292058 & -1.244912965060674 & 1.580288612473559 \\ \text { Al } & -1.529732042616033 & -3.930359961363529 & 1.990232815865880 \\ \text { Al } & -0.077177051550805 & 3.097212082846887 & 1.921012754288045 \\ \text { Al } & 0.321795830027478 & -2.207514015756094 & 2.896444179387792 \\ \text { Al } & -0.402108715198768 & 0.492871207204681 & 2.911142031819303 \\ \text { Al } & 1.968936137725521 & -0.310944794414389 & 3.977115038993714 \\ \text { Al } & 1.281822607240141 & 2.283906629159727 & 4.089242156530998\end{array}$




$\begin{array}{lrrr}\text { 2.8 } & \text { Al_27 } & & \\ \text { 27 } & & & \\ \text { Al } & -0.729972903416934 & 0.051191731730603 & -3.652518869609882 \\ \text { Al } & -1.833601492497438 & -3.963868559194810 & -1.552264963279044 \\ \text { Al } & 0.787429330195128 & 2.140411459437814 & -3.924882817576236 \\ \text { Al } & -0.030504062560370 & -2.028324198600061 & -2.002115867469147 \\ \text { Al } & -2.476518902489318 & -1.937387226980463 & -3.322412346090994 \\ \text { Al } & 1.681421598559602 & -0.012004254169101 & -2.353872188603862 \\ \text { Al } & -0.755645024854486 & 2.153156256313910 & -1.768960625648887 \\ \text { Al } & 2.027147246239759 & 2.624991267270104 & -1.695692719389744 \\ \text { Al } & -2.263607004118568 & -0.071087674714011 & -1.378576862882770 \\ \text { Al } & -3.886850889775681 & -2.197415245355524 & -0.991950423476760 \\ \text { Al } & 0.482761122505865 & -4.040072456756921 & -0.157754474510627 \\ \text { Al } & 0.099545610586196 & -0.141363561940334 & 0.045166100906236 \\ \text { Al } & 2.252224045382750 & -2.008229998623601 & -0.532430764401912 \\ \text { Al } & 2.786889915606045 & 0.600497183894170 & -0.004183387493443 \\ \text { Al } & -1.573981316842049 & -2.313423569485248 & 0.549085497730886 \\ \text { Al } & -2.117787957227561 & 1.903061143883610 & 0.479457118969760 \\ \text { Al } & 0.521640748601779 & 2.529963038826029 & 0.597133853963797 \\ \text { Al } & 3.116606806898345 & 3.284654801795167 & 0.647416622644364 \\ \text { Al } & -3.554612629425213 & -0.468323062521366 & 1.011500568715098 \\ \text { Al } & 0.785393625130418 & -2.278326830322540 & 1.892975137038432 \\ \text { Al } & -1.279138156041713 & -0.556623444035065 & 2.535226852789702 \\ \text { Al } & 3.347333410204250 & -1.365698376039774 & 1.874424837265808 \\ \text { Al } & 1.241728062335412 & 0.317301387771710 & 2.546253771123414 \\ \text { Al } & 3.753614172717208 & 1.253111236453163 & 2.413741856753340 \\ \text { Al } & -3.288426491786304 & 1.391662622628694 & 2.802959446646666 \\ \text { Al } & -0.798148947708869 & 2.106080821911476 & 2.966368038424658 \\ \text { Al } & 1.705060083781749 & 3.026065506822356 & 2.975906607461102\end{array}$




$\begin{array}{llll}\mathbf{2 . 9} & \text { Al_28 } & & \\ \text { 28 } & & & \\ & & & \\ \text { Al } & 1.968660359780511 & 0.395161660166940 & -2.396916656190634 \\ \text { Al } & -2.954572153901250 & -1.495581647192072 & -3.221871477312488 \\ \text { Al } & -0.433525647363364 & -0.531407799583340 & -2.844129639158764 \\ \text { Al } & -2.713899064996837 & 0.992726404146676 & -2.437915021807872 \\ \text { Al } & -0.181782086499354 & 1.981203974506052 & -1.899460570026360 \\ \text { Al } & 1.265451687816853 & -2.019817778686984 & -1.372503130719915 \\ \text { Al } & -1.266876843967653 & -3.008971608051977 & -1.908254708331541 \\ \text { Al } & 4.295850936139832 & 1.337906003198112 & -1.612791141799505 \\ \text { Al } & -2.436230265589599 & 3.521076524194370 & -1.611430743411053 \\ \text { Al } & 2.239105088180612 & 2.961600074288233 & -1.085470351873552 \\ \text { Al } & 0.061769325135892 & 4.494514562508583 & -0.914310833041249 \\ \text { Al } & -2.655857772501116 & -1.033494023786936 & -0.553407953205239 \\ \text { Al } & 0.471388104615940 & -4.515931117105588 & -0.549865843842074 \\ \text { Al } & 3.684809854346396 & -1.039907804075224 & -0.554962939708741 \\ \text { Al } & -0.010726291876393 & -0.008674902489338 & -0.034959245393072 \\ \text { Al } & -2.276345578509611 & 1.512157671502719 & 0.247466692055621 \\ \text { Al } & 2.553002524879203 & 1.019753125895440 & 0.747248003852553 \\ \text { Al } & 0.393434299945612 & 2.564562711339734 & 0.961506579026395 \\ \text { Al } & -0.828773783030927 & -2.497617579770858 & 0.778149121464663 \\ \text { Al } & -1.859556067763900 & 4.061099479752142 & 1.049109123844209 \\ \text { Al } & 2.951105221028033 & -3.507111460348034 & 0.143742143410890 \\ \text { Al } & 1.836660614205726 & -1.437173530523022 & 1.491368833355313 \\ \text { Al } & -2.268855888248574 & -0.553474424936402 & 1.991534085598262 \\ \text { Al } & 1.027728302510035 & -3.945126503166433 & 2.108175259147171 \\ \text { Al } & 0.599299210747565 & 0.577177654361337 & 2.730167798101720 \\ \text { Al } & -1.612164969173708 & 2.109390586091989 & 2.851397541328126 \\ \text { Al } & -0.158291194239187 & -1.922231758967897 & 3.384485443322319 \\ \text { Al } & -1.690807921670738 & -0.011808493268211 & 4.513899631314819\end{array}$




\subsection{Al_29}

29

$\begin{array}{lrrr}\text { Al } & -2.128378260979966 & -0.440914962218839 & -3.786902108828111 \\ \text { Al } & 0.011462219120478 & 1.306890759017893 & -3.457402527940071 \\ \text { Al } & 2.195255852912073 & 2.825977567879489 & -3.004038395670577 \\ \text { Al } & -1.647739474319252 & -3.147029679954036 & -2.889292076345215 \\ \text { Al } & -4.422768355422162 & 0.301244684831222 & -2.408559885355727 \\ \text { Al } & 0.148466000302117 & -1.257135309050163 & -2.460794410761399 \\ \text { Al } & 0.840550503591222 & -4.005305806100899 & -2.096471344518847 \\ \text { Al } & 2.185636303886357 & 0.420825432261076 & -1.935132951653242 \\ \text { Al } & -2.083860347824498 & 1.405591862735605 & -1.698408207483228 \\ \text { Al } & -2.715151934304025 & -1.365676190247702 & -1.199219589380829 \\ \text { Al } & 0.162921585375157 & 2.652613694759740 & -1.109298450632182 \\ \text { Al } & 2.191281728597335 & -1.927489628931908 & -0.806307577389628 \\ \text { Al } & 2.856726453102976 & 2.710983552238535 & -0.402383279143240 \\ \text { Al } & -0.791219733002364 & -2.840138338693492 & -0.250628328853510 \\ \text { Al } & -0.132762927851731 & 0.001772033336593 & -0.055546454529715 \\ \text { Al } & -3.854185832073336 & 0.597185873293027 & 0.254000936655494 \\ \text { Al } & 1.473044474694188 & -4.115567230094621 & 0.522557710794771 \\ \text { Al } & 2.593294872389547 & 0.166704114507509 & 0.728433562437376 \\ \text { Al } & -1.684051544105290 & 2.198013923652795 & 0.822593438906102 \\ \text { Al } & 0.856778192414717 & 2.333137173666717 & 1.428302806103323 \\ \text { Al } & -2.117759275879060 & -1.320910653889927 & 1.473678771386941 \\ \text { Al } & 3.238134869448590 & -2.454519124645142 & 1.807538029786185 \\ \text { Al } & 0.559601763084926 & -1.905146123693266 & 1.850073110829110 \\ \text { Al } & 3.441634108578743 & 2.251463545971056 & 2.220090717695797 \\ \text { Al } & -3.125735659541575 & 0.892392378387145 & 2.835257451565498 \\ \text { Al } & -0.439320481032574 & 0.269792670819207 & 2.827002079519024 \\ \text { Al } & 2.284500748329343 & -0.344544834953746 & 3.318784503934596 \\ \text { Al } & 1.313371797066505 & 2.034531554864287 & 4.019636655539315 \\ \text { Al } & -1.209727646558421 & 2.755257060251825 & 3.452435813331979\end{array}$




\subsection{Al_30}

30

$\begin{array}{lrrr}\text { Al } & 0.881568511723474 & 0.070655135789744 & -3.878262665466415 \\ \text { Al } & -0.664233206619651 & 2.582378216448971 & -3.089607168828661 \\ \text { Al } & -1.726085868102536 & 0.456632912649159 & -4.224197547773762 \\ \text { Al } & 2.063630029699462 & 2.339981503311222 & -2.865073706024676 \\ \text { Al } & -3.449238064584084 & -0.955008445458171 & -2.770984526881142 \\ \text { Al } & 0.715014681373487 & -2.241953991938430 & -2.514019543321493 \\ \text { Al } & -1.954630327002677 & -2.922965910002024 & -1.844797005641716 \\ \text { Al } & -3.006333038042778 & 1.432297120808242 & -1.808450213615561 \\ \text { Al } & -0.860679098370364 & -0.205586380834726 & -1.600726806255257 \\ \text { Al } & 1.882236165291692 & -0.002109583912432 & -1.423159295472177 \\ \text { Al } & 0.059573934647661 & -4.477739716359872 & -1.065300335939657 \\ \text { Al } & -1.975214858761119 & 3.728708876114695 & -0.932527496238777 \\ \text { Al } & 0.348544692002207 & 2.298444715689380 & -0.604685658902058 \\ \text { Al } & 3.088351345345545 & 2.206710157208528 & -0.425509402143000 \\ \text { Al } & 1.795026720366222 & -2.326061081623907 & -0.056677888457273 \\ \text { Al } & -3.116218433053360 & -0.909465578988204 & -0.116092545201562 \\ \text { Al } & -0.910471254147824 & -2.438195660522726 & 0.570204382018700 \\ \text { Al } & -1.923030545791171 & 1.391772928860657 & 0.612507081286854 \\ \text { Al } & 0.360598506954162 & -0.008113381291775 & 0.956715791458738 \\ \text { Al } & 3.150513553010123 & -0.206266353997650 & 1.028421235931710 \\ \text { Al } & 0.729143610783305 & -4.313286513564929 & 1.592451821555532 \\ \text { Al } & -0.941875077700219 & 3.796197731116282 & 1.492319242764871 \\ \text { Al } & 1.524413818407353 & 2.908134957459119 & 1.796897225639610 \\ \text { Al } & 4.032759059921796 & 2.142867448813222 & 2.153662912362179 \\ \text { Al } & -2.034609296088661 & -0.806146029958185 & 2.378184310263860 \\ \text { Al } & 1.585495581309026 & -1.902456971998199 & 2.535947828651981 \\ \text { Al } & -0.720958047171461 & 1.487157283788720 & 3.038449318041092 \\ \text { Al } & 1.990500101290333 & 0.671749155328778 & 3.304389070030894 \\ \text { Al } & -0.865565554634687 & -3.070524807697399 & 3.338867307415706 \\ \text { Al } & -0.058227642055279 & -0.727807735238073 & 4.421054278741432\end{array}$




\subsection{Al_31}

31

$\begin{array}{lrrr}\mathrm{Al} & 0.708787611284013 & -1.230551523064696 & -2.469245962379290 \\ \mathrm{Al} & 2.575595354226735 & 0.433053351486643 & -3.334671682065337 \\ \mathrm{Al} & 0.254238203805901 & 1.439720984785383 & -2.409884436070056 \\ \mathrm{Al} & -1.329142538480056 & -2.937068578361913 & -1.946102388845816 \\ \mathrm{Al} & 2.533099984600616 & 2.805164816255616 & -1.882789494483561 \\ \mathrm{Al} & -1.689204600512022 & -0.234828698819417 & -1.697259789173422 \\ \mathrm{Al} & 4.672519509228687 & 0.760307917828008 & -1.655134385539768 \\ \mathrm{Al} & -2.081434162899650 & 2.455744799755889 & -1.530112106692224 \\ \mathrm{Al} & 0.052574940094798 & 4.029845046852921 & -1.496816686415030 \\ \mathrm{Al} & -3.902262802818930 & -1.758724493743015 & -1.547480564658239 \\ \mathrm{Al} & 3.345504506611737 & -1.667535994834702 & -1.484826363262331 \\ \mathrm{Al} & -4.230072236160512 & 0.862878333559438 & -1.228197515993820 \\ \mathrm{Al} & 1.276272458473375 & -3.371776568945472 & -0.901927855959258 \\ \mathrm{Al} & 1.920344921660744 & 0.403298517792779 & -0.506558450891474 \\ \mathrm{Al} & -0.707888100708479 & -4.669648407359550 & 0.260364098284301 \\ \mathrm{Al} & -0.529565641339952 & -1.656478078946630 & 0.311318583247488 \\ \mathrm{Al} & 3.780541265815447 & 2.248843958757385 & 0.493972932886445 \\ \mathrm{Al} & -0.793904520054939 & 1.087751031378575 & 0.531745804831779 \\ \mathrm{Al} & -2.895153198157868 & -3.219867206282081 & 0.502320325233757 \\ \mathrm{Al} & 1.228006827729304 & 2.955612954780394 & 0.695298790046010 \\ \mathrm{Al} & -3.042397432170222 & -0.515007610329318 & 0.771247714159035 \\ \mathrm{Al} & -1.354810298784599 & 3.855541346576864 & 0.903815606759490 \\ \mathrm{Al} & 4.271365927596479 & -0.371104381487978 & 0.880002858067568 \\ \mathrm{Al} & -3.481408401754265 & 2.175589383570328 & 1.139671099200285 \\ \mathrm{Al} & 2.093472484437257 & -1.748896068368079 & 1.395746888006864 \\ \mathrm{Al} & 2.102374040236345 & 0.916061459010731 & 2.164125897222496 \\ \mathrm{Al} & 0.188679231733689 & -3.306915209771034 & 2.415610233139345 \\ \mathrm{Al} & -0.024557290004356 & -0.564981837756369 & 2.699573837429719 \\ \mathrm{Al} & -2.243383093815300 & -2.073424514091212 & 2.855896696121919 \\ \mathrm{Al} & -0.235745890555968 & 2.213264630917990 & 2.928966171992744 \\ \mathrm{Al} & -2.462447059318009 & 0.684130638852494 & 3.141330145800362\end{array}$




\subsection{Al_32}

32

$\begin{array}{lrrr}\text { Al } & -0.091651783915736 & -3.488049905533137 & 2.080477719728476 \\ \text { Al } & -0.266510392201306 & -0.399717553765274 & 4.144445545897872 \\ \text { Al } & -1.877113473541417 & -1.996393878753715 & -4.574300448644244 \\ \text { Al } & -2.178337953248363 & 0.355515233285114 & -2.966586571131538 \\ \text { Al } & 2.199902756664265 & 2.762636292246338 & -1.236587248606588 \\ \text { Al } & -0.275460593007566 & 4.200840085463836 & 1.659386422288318 \\ \text { Al } & 0.208321330028213 & -3.035139311044789 & -3.088786581403158 \\ \text { Al } & 2.368057685505292 & -1.897895755303637 & -4.344576076656026 \\ \text { Al } & -0.203181961333707 & 4.182728799204483 & -1.221454231001324 \\ \text { Al } & -2.470693205831557 & 0.309339515492848 & 2.523109954577887 \\ \text { Al } & 2.197006682175518 & -2.185002187249372 & 1.043235584654418 \\ \text { Al } & -2.399743404915659 & -2.401369907424343 & 0.755701490436779 \\ \text { Al } & 2.064387349840056 & 0.302988955206734 & 2.730605735928131 \\ \text { Al } & 0.192606983211656 & -0.234179147713515 & -4.237385809466016 \\ \text { Al } & 0.071556008280700 & 2.034921283434564 & -2.846779434069552 \\ \text { Al } & 2.101983373314260 & 2.686197555039582 & 1.561964600305874 \\ \text { Al } & -2.255384218940092 & -2.175923786107688 & -1.868024094644724 \\ \text { Al } & 2.384334912712429 & -2.021863219562281 & -1.585733999502546 \\ \text { Al } & -2.449571914859204 & 2.659784234710573 & 1.240984190738317 \\ \text { Al } & 0.025559093804755 & -3.294864320295940 & -0.504900177485460 \\ \text { Al } & -0.237411962197962 & 1.967966630953981 & 3.043590166178552 \\ \text { Al } & 2.385184987531686 & 0.468281476851869 & -2.708053451547300 \\ \text { Al } & -2.318589877831055 & 2.610516551334575 & -1.492544044104644 \\ \text { Al } & -2.315859353631772 & 0.168134021288532 & -0.208861382680045 \\ \text { Al } & 2.202922839283959 & 0.345960065600883 & 0.017886943177253 \\ \text { Al } & -0.114796998452910 & 1.754721079937203 & 0.129855089698482 \\ \text { Al } & 0.027651739781433 & -0.593060548668300 & -1.470562282558872 \\ \text { Al } & -0.165391789055729 & -0.765156905362067 & 1.374498464595041 \\ \text { Al } & 1.818471430318890 & -2.177843621086925 & 3.634524193549007 \\ \text { Al } & -2.244788236096563 & -2.191673078965222 & 3.343068113093068 \\ \text { Al } & -0.297824209136740 & -3.117557355597104 & 4.787904223580187 \\ \text { Al } & 1.914364155744252 & 5.165158702382218 & 0.283897395074348\end{array}$




\subsection{Al_33}

33

$\begin{array}{lrrr}\mathrm{Al} & 0.650617869267137 & -0.252916929973676 & -4.240450873506258 \\ \mathrm{Al} & -0.503980320029875 & -2.667965498359179 & -3.776107139505211 \\ \mathrm{Al} & -0.880627735606124 & 1.740014396608689 & -3.262634579216585 \\ \mathrm{Al} & 2.682080014665997 & -0.896998546966751 & -2.561528216962873 \\ \mathrm{Al} & -2.258448759384247 & -0.857392800215715 & -2.900885039160990 \\ \mathrm{Al} & 1.557252641905301 & 1.514619018849791 & -2.109244871634606 \\ \mathrm{Al} & 1.432675754907310 & -3.242096638865539 & -1.996751197050518 \\ \mathrm{Al} & -2.510780789975382 & 3.429270046828128 & -2.008712955646382 \\ \mathrm{Al} & 0.146677220130405 & -0.885311978647454 & -1.543457646359300 \\ \mathrm{Al} & -1.436026794305230 & -3.183805202057383 & -1.316166925146238 \\ \mathrm{Al} & -3.820688662849577 & 1.055625774756663 & -1.910403277527191 \\ \mathrm{Al} & 0.017050231747145 & 3.498107679568834 & -1.127845260657473 \\ \mathrm{Al} & 3.637110622334049 & 0.767898159193777 & -0.577798151138072 \\ \mathrm{Al} & 2.421844993582495 & 3.221565576426201 & -0.016151787703857 \\ \mathrm{Al} & -1.390290622713624 & 1.062922977579509 & -0.615264312424788 \\ \mathrm{Al} & 2.415389156231830 & -1.577591600061815 & 0.044007408919585 \\ \mathrm{Al} & -3.117774263160652 & -1.288028804803579 & -0.363944368672358 \\ \mathrm{Al} & 0.818904876395138 & -3.728875974343579 & 0.547768730221916 \\ \mathrm{Al} & 1.114168773871500 & 0.860327339049492 & 0.653673084567346 \\ \mathrm{Al} & -2.256182366657323 & 3.299131832384074 & 0.657502611711042 \\ \mathrm{Al} & -2.246283400045062 & -3.543979971428013 & 1.166638954902140 \\ \mathrm{Al} & -0.546155494469399 & -1.366574541690029 & 1.089602805951936 \\ \mathrm{Al} & 4.764966798947498 & 2.374475585045991 & 1.253384746794977 \\ \mathrm{Al} & -3.765761457767328 & 1.041067692788474 & 0.795462416855665 \\ \mathrm{Al} & 2.513733294677431 & 2.634651347472573 & 2.683735404673561 \\ \mathrm{Al} & -3.007717487857729 & -1.225634831506701 & 2.329453496673363 \\ \mathrm{Al} & -1.496701637982474 & 1.041725762937613 & 2.216062044091220 \\ \mathrm{Al} & 0.128514423229447 & 3.187730631156370 & 1.790093041555529 \\ \mathrm{Al} & 3.386466539676645 & 0.091510748802319 & 2.111453269650431 \\ \mathrm{Al} & 1.756588296049950 & -1.939961839596496 & 2.640383742616658 \\ \mathrm{Al} & -0.261899401608501 & -3.656554593563964 & 2.971787411921857 \\ \mathrm{Al} & 0.892396694392090 & 0.717539045303500 & 3.438178448557107 \\ \mathrm{Al} & -0.837119007598830 & -1.224493862672119 & 3.938158982648362 \\ & & & \\ & & \end{array}$




\subsection{Al_34}

34

Al $\quad-0.208909008009144$

0.618185771775696

$-3.644036212665020$

Al $\quad 2.398911892151920$

1.106880562712949

$-3.867445022702866$

Al -0.639977770573052

$-2.116173017653392$

$-2.937458109104309$

Al $\quad-2.715316169714209$

$-0.013559461461334$

$-3.138184800786533$

Al 1.916852639748829

$-1.519072833484232$

$-3.462418171970583$

Al 3.892208014630352

1.262822467799028

$-1.632141094473233$

Al $\quad-3.109716903546364$

$-2.561274135082980$

$-2.179400105529874$

Al 1.069353995105372

2.832901726798966

$-2.303283168898118$

Al -1.537300684342097

2.229574505464495

$-1.898375627883601$

Al $\quad-5.129803670049934$

$-0.821183496819854$

$-1.943720984488730$

Al 1.067612062059260

$-2.489768156264651$

Al 3.480909304344374

$-1.359194882670282$

$-0.853080672919907$

Al 1.305485484118682

0.262256397661233

$-1.232284377820326$

Al -0.181222679044643

4.370495307115613

$-1.307109896607296$

Al -3.657909255133466

1.075336175450865

$-0.520772210106852$

Al -1.331602703596987

$-0.471034610582974$

$-0.726342485508255$

Al 2.612123717268494

2.762098922848590

$-0.823950367652809$

Al 2.776269349673115

$-2.411131085749831$

0.163016129360226

1.237812916640372

Al -1.451746659329038

$-3.247381313153071$

$-0.279269081891620$

Al $\quad 0.046680608657896$

1.789195849503178

Al $\quad-2.310027471566589$

3.137050976041213

Al $\quad-3.672791665497447$

$-1.507927242155453$

0.522205074042452

0.551287244992292

0.312481725912248

Al 1.073670481719182

4.164663443529951

0.231067121371717

Al 3.155283636770033

$-3.616268100292602$

1.853629931428063

0.954275225741668

Al 0.301265739404364

$-0.838895505357543$

1.649936425149383

Al 0.467367170827432

0.639772017821270

1.322401535279754

Al $\quad-2.284888325035650$

$-3.551478464254196$

1.559058449064354

Al 2.249920963298626

$-1.938526287209921$

3.619933544885225

Al $\quad-1.962745126711837$

Al 2.213065602484894

$-0.748706273450843$

Al -1.028929343288308

2.869000203320775

Al 1.772290061072392

1.753509234966506

Al -0.539967888725929

0.325424752255644

2.265014083170207

3.425001320475536

2.945357295084650

2.736795932585570

3.620525514872110

Al -0.036415399170535

$-2.218660570794492$

4.010540042325822 


\subsection{Al_35}

35

\begin{tabular}{|c|c|c|}
\hline-0.538233276045817 & 0.971734771859058 & \\
\hline 1.910592060712272 & 0.029393907380268 & -3.727666691275817 \\
\hline-2.658556368460377 & -0.587062517234557 & -2.490405752474440 \\
\hline-1.101408318013016 & 3.655134346409216 & -2.055483021882206 \\
\hline-0.162195938834316 & -1.649071433347116 & -2.834367260867475 \\
\hline-2.956830651331162 & 2.003255513014961 & -3.059567353531734 \\
\hline 4.519450674584887 & -0.885747919975602 & -3.136785511796102 \\
\hline 1.336305911592508 & 2.627194891784582 & -2.221677303442179 \\
\hline 2.404565877916733 & -2.214499404861276 & -2.106104634194828 \\
\hline-2.301189446946436 & -3.06 & -1. \\
\hline 3.712688037181611 & 1.501322702791988 & -2.247214157835963 \\
\hline-1.255870268407383 & 560 & -0.8 \\
\hline 0.380283145666175 & -3.702714 & -1.1241 \\
\hline 1.283535076147189 & 02 & \\
\hline 3.9560 & -0.5 & -0.4 \\
\hline-1.548965947116013 & 243620 & -0.1 \\
\hline-3.5 & -0.9 & \\
\hline 5917772210 & -1.427289 & -0.0 \\
\hline 2.8937 & 2160624 & $0.1^{\prime}$ \\
\hline 0.4248066027 & 01 & 0.2 \\
\hline-2.313946653579444 & & \\
\hline 1.800954632518170 & 91316388 & 0.54900 \\
\hline-4.010000124532089 & 1.514743467952041 & -0.644989 \\
\hline-2.938729584489874 & -3.3 & 0.9 \\
\hline 0.288779837371607 & 0.611792507467333 & 1.520822 \\
\hline-2.530466859013276 & 1.00 & 1.717005925050561 \\
\hline-0.261529731435482 & -3.670824458602731 & 1.615812642969532 \\
\hline 2.967596612040520 & 955280442996 & 1.976042822171440 \\
\hline 1.919507352956119 & 2.570351093690748 & 2.557902707970038 \\
\hline-1.999637079122836 & -1.5097792988 & 2.55009 \\
\hline-0.883943313010812 & 2.951058983532166 & 2.652861278305817 \\
\hline 0.775993953316663 & -1.697207446485848 & 3.037650556955755 \\
\hline-0.973656391885891 & 0.442201606438058 & 3.960428479211942 \\
\hline 1.784311070419434 & 0.389077499603683 & 4.269144598750845 \\
\hline 0.480935137218552 & 2.602342464122041 & 4.938484571958774 \\
\hline
\end{tabular}




\subsection{Al 36}

36

Al 1.303807163274493

Al 1.459504743658870

Al 0.787080591996421

Al -0.921798964782944

Al -1.482877600585736

Al $\quad-2.007207450164802$

Al 0.259940506441929

Al $\quad-2.055767891868165$

Al 2.693935416261315

Al 2.592064431252284

Al 2.092468103154268

Al -2.363663231459701

Al 0.192036771246094

Al $\quad-2.989524335612063$

Al 0.093520614214453

Al -0.398336866053093

Al -2.790206844526590

Al $\quad-0.215989779094635$

Al 3.844983810979846

Al 3.806189339182444

Al 1.459234986866681

Al $\quad-3.720986076398541$

Al 1.451620832661689

Al -1.251023764204939

Al $\quad-1.222989097855477$

Al 1.493231545684962

Al -3.608346626030078

Al -1.155251190547848

Al $\quad 5.127913231801390$

Al 3.151889005835837

Al $\quad-4.532082597523872$

Al 2.633743846685162

Al -2.491085102382153

Al 0.612199179353871

Al 0.082728689640613

Al -1.930955391102045
1.450344834560378

4.239303428488414

$-1.099992826376580$

3.074796154788009

0.506889240626307

$-2.050570066362720$

$-3.638929070686460$

$-4.747676705496916$

0.014149990720879

2.757627942281479

$-2.611732950843209$

2.380675180513609

1.261321146082452

$-0.267993299906173$

4.019318982752749

$-1.439501770596832$

$-2.925665643468113$

$-4.125460735361177$

$-1.491279975103964$

1.353209823575153

$-0.203033845383413$

1.594972365842391

2.647678872301631

0.378018516867440

3.171013579346775

$-2.939075148008362$

$-1.184082038053667$

$-2.395502135380662$

$-0.007049745608876$

$-1.641777786500340$

0.516073061990141

1.079203360702888

2.207006621430088

$-1.064443588943105$

1.660944612370349

$-0.478780383160490$
$-3.646114239940347$ $-3.397987634687738$ $-3.319737024825664$ $-3.356593957120663$ $-3.226703572066469$ $-2.976854258701393$ $-2.871895731806346$ $-2.209352957983109$ $-1.719154908167373$ $-1.470709813702607$ $-1.287144984727581$ $-1.267201644577314$ $-1.122147852868622$ $-1.088842005448093$ $-0.958661667900763$ $-0.812962433572313$ $-0.383633576564549$ $-0.186418327382096$ 0.310373154747328 0.418436391270795 0.848726301844847 0.834610929265176 1.036219163690395 1.085937960201269 1.144776010525309 1.410247710163736 1.436114511982943 1.771966659111534 2.314414021387838 2.988455428376264 3.292831037004868 2.997771807734338 3.240129910634360 3.367488790253537 3.178932747555665 3.624684056292825 


\subsection{Al_37}

37

\begin{tabular}{|c|c|c|c|}
\hline Al & 1467587870505 & -1.762430639158910 & \\
\hline Al & 0.065827186230139 & 0.468478512221811 & -3.638620198300393 \\
\hline 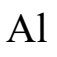 & 3.714931006947596 & -2.485513423250991 & -3.508581880708847 \\
\hline $\mathrm{Al}$ & 2.884740321916066 & 0.134650726564411 & -2.967487460769646 \\
\hline A & -1.201932445006975 & 2.624056153539014 & -2.840933907974335 \\
\hline & -0.960401630249494 & -2.221563364366165 & -2.959662921589810 \\
\hline $\mathrm{Al}$ & 1.585170166421609 & 2.260436065104095 & -2.094676307487558 \\
\hline & -2.170235320498493 & 0.081461546873109 & -2.166390150113759 \\
\hline & 1.512522855051238 & -3.371544567406096 & -1.995686401063604 \\
\hline $\mathrm{A}$ & -2.397532252730887 & 4.798996519021300 & -1.708717153086521 \\
\hline $\mathrm{Al}$ & 0.170951084839961 & 4.292533804336996 & -1.078480601171355 \\
\hline A & 0.848040945138179 & -0.767186740272866 & $-1.32139394 \mathrm{C}$ \\
\hline 1 & -3.150610803370349 & 536617409 & 285 \\
\hline $\mathrm{Al}$ & 3.562418954767988 & -1.777134039199479 & -0.9479422 \\
\hline 1 & -3.2006617 & 2.3166 & 5602 \\
\hline $\mathrm{Al}$ & 3.115501242428769 & 0.811771816025487 & $-0.30088 c$ \\
\hline $\mathrm{Al}$ & -0.492832435119674 & 671457269 & -0.40056 \\
\hline $\mathrm{Al}$ & -0.686674859437518 & -3.622905977451593 & -0.56612 \\
\hline $\mathrm{Al}$ & -4.024309986543248 & -0.06706940514 & -0.11871 \\
\hline Al & 1.7606624 & 2.884 & 0.57224 \\
\hline A & -1.385849118981140 & -1.052628927225761 & 0.251397337268282 \\
\hline $\mathrm{Al}$ & -1.65 & 3.708 & 0.84180 \\
\hline $\mathrm{Al}$ & -5.117541597955740 & -2.405308281154284 & 0.53733 \\
\hline $\mathrm{Al}$ & 313551278560 & -2.669175746271323 & 0.66384 \\
\hline $\mathrm{Al}$ & 0.970720548633128 & -0.004686273359514 & 1.37498 \\
\hline Al & -2.850871314434176 & -3.665371574775271 & 0.956024253149044 \\
\hline $\mathrm{Al}$ & 3.586236213266021 & -1.171426860038359 & 1.648239268439527 \\
\hline $\mathrm{Al}$ & 0.544060183645847 & 4.921165285267364 & 1.85817158 \\
\hline $\mathrm{Al}$ & -2.416260797283754 & 1.272736038372424 & 1.652920465844170 \\
\hline $\mathrm{Al}$ & -0.717644039844323 & -2.863313439621681 & 1568 \\
\hline $\mathrm{Al}$ & 1.481212738858947 & -1.954102713672244 & 3.351935479993184 \\
\hline $\mathrm{Al}$ & -0.993768635720219 & -0.288484281863141 & 3.360157058169435 \\
\hline $\mathrm{Al}$ & -0.020012038383467 & 2.269441140365279 & 2.608805260646617 \\
\hline 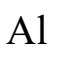 & 1.300846334027581 & 0.620839843015583 & 4.282631297975000 \\
\hline $\mathrm{Al}$ & 3.075852221696016 & 1.463468203448807 & 2.435815909498006 \\
\hline $\mathrm{A}$ & 3.692764275228754 & -0.601726291236176 & 4.302563573443244 \\
\hline$\Delta$ & -3.226562197925146 & -1.273434202804102 & 2.316767135090416 \\
\hline
\end{tabular}




\subsection{Al_38}

38

Al 1.971522221702559

Al -2.670097641729762

Al -0.694168376173190

Al 1.214726539651430

Al $\quad-0.105583422825454$

Al 3.849960174420533

Al $\quad-2.350979298597703$

Al 2.303832787574386

Al -4.608896606206741

Al 0.198733675387947

Al 3.634554294277457

Al -3.919244966188391

Al -2.165773867935512

Al 0.579998428607889

Al -0.422371541674540

Al -2.057554114814183

Al -1.316285724546615

Al 2.259754362616018

Al 2.602783696803526

Al 0.334235424187989

Al 3.942271671768715

Al 1.157355029536795

Al $\quad-3.531879100680072$

Al $\quad-3.412670445985589$

Al 2.355034470793113

Al -1.945414231081504

Al -0.910276726504138

Al 0.747425922487672

Al -0.568839854483425

Al $\quad 4.229667375038487$

Al $\quad 1.585952819401610$

Al 2.924768442532789

Al -2.312943472331325

Al 0.348243568074940

Al $\quad-3.028254823738735$

Al 1.432582123015584

Al -0.716503831536246

Al -0.935664980846335
1.261204940437686

0.455430818923874

2.146866741558634

3.840622363377786

$-0.481245747587389$

3.395051879480169

$-2.094706561081195$

$-1.122736862751662$

$-3.012430449051241$

$-2.688199434353888$

1.148545975199896

$-0.342291489278971$

1.712323156040998

1.178583713034907

3.758745302242804

$-3.750619090370882$

$-0.735462592542445$

3.355273531940917

$-3.336104557607677$

$-4.715343460329046$

$-0.992631549787198$

$-1.156423358015045$

$-1.941301660934175$

0.799224319336984

1.165866846850060

3.181078400461523

$-2.599889440905611$

3.270402221147213

0.899584022508083

$-3.222793860888467$

$-3.319410242953187$

$-0.950650604448338$

$-0.805620870126148$

$-1.277962053733159$

1.855130294678517

1.265219928575659

3.198195272670654

0.658474158279338
$-3.541348054873320$

$-3.775636298489649$

$-3.459895497154189$

$-3.038877427980128$

$-3.244403541431219$

$-2.870521709711163$

$-2.937823109513090$

$-2.233675350521561$

$-1.572915047794443$

$-1.874703371709781$

$-1.420788336017967$

$-1.466956954485677$

$-1.217562401414812$

$-1.025682957256473$

$-0.897734401165781$

$-0.796638803054559$

$-0.569081996680866$

$-0.578063046254380$

$-0.699175948269911$

$-0.016176626326546$

0.060055668998130

0.420641507670998

0.718961923950632

0.961874257747210

1.049535566721326

1.223346978549268

1.400164936507029

1.615321577548713

1.728795093511250

1.515956928089613

2.055536648944482

2.657555977472100

2.878648898351432

3.355344866869762

3.390789730876010

3.582230566472639

3.867251090098227

4.755648661726676 


\subsection{Al_39}

39

Al $\quad 0.769185795776082$

Al 0.562365813929331

Al 0.643248010713240

Al -1.628073725303466

Al -1.761421919849509

Al 2.122335186339868

Al $\quad-3.913342616384819$

Al 2.938181349555569

Al 2.552162903823822

Al $\quad 0.560659245976680$

Al -0.447806144532604

Al $\quad-1.554874496808978$

Al 0.424791291355314

Al 2.530480692410402

Al 4.992318998751617

Al -3.774327665599226

Al -2.751980143538001

Al $\quad-5.992256690819710$

Al 0.118095829841973

Al 4.741858270058747

Al $\quad-2.012346902642344$

Al 1.846786544721137

Al $\quad-4.986839962046621$

Al 2.780345800460218

Al 0.387736588439658

Al $\quad-0.637427840808844$

Al $\quad-2.025999591563062$

Al $\quad 2.356842193504370$

Al $\quad-4.165371999736382$

Al 4.796081406915514

Al 0.237271973362985

Al $\quad-2.943432157901888$

Al 1.667279508450672

Al 0.121822633362614

Al $\quad-2.050207464566105$

Al 2.562749380729741

Al -0.816937858275296

Al 0.175090162058591

Al $\quad 1.574957599838797$
$-2.436949627720938$

0.146926749796366

2.692477138664451

$-1.500481679520792$

1.168847573880502

$-4.418387037875296$

$-0.466341011293498$

$-1.794086406386130$

0.898445052842343

5.226402121689068

$-3.777826861642934$

3.621709036922855

$-1.225727562634903$

3.604423632298900

$-0.316723677173243$

2.201019397437260

$-2.848716936582888$

0.632166929725912

1.457366817294233

2.352972380194885

$-0.200708282316436$

$-3.507690202165733$

$-1.675301528356135$

$-1.010652222836788$

3.927074618835308

$-2.963590256443212$

2.440228265586830

1.849711217369730

0.748960889713517

0.621298467732354

$-0.325039204862932$

$-1.925034013574107$

$-2.641885101895272$

2.271037103561680

0.534205651986737

0.005011595862085

$-1.999388189318214$

0.416207764974587

$-1.781962603770186$
$-3.715233198288293$

$-3.450914997573470$

$-2.885606623633043$

$-2.889293993410174$

$-2.585864975340890$

$-2.576290476607337$

$-2.081657760830405$

$-2.074668441041549$

$-1.664505835909798$

$-1.779205512350982$

$-1.598367038009821$

$-1.373921050208356$

$-1.004542865326867$

$-1.073074598905455$

$-1.048719710459787$

$-1.039503351792050$

$-0.705986858119765$

$-0.651457046928334$

$-0.497046800254711$

$-0.473260448221499$

$-0.074365510281636$

$-0.047727421088618$

0.224718587322169

0.552678149996543

0.656407132213266

0.984445648257365

1.058188642769142

1.064345057351730

1.401894464655907

1.651615406297559

1.590479673593398

1.955107374014286

2.441065430152506

2.655518879667515

2.956837043654431

3.095044946596079

3.516004516351201

4.486150470731562

5.000713090958191 


\subsection{Al_40}

40

Al $\quad 2.779760244010900$

Al 0.856452628679085

Al 0.182768601801994

Al -1.740992738761616

Al 2.134977352907550

Al -0.448845116907357

Al -2.426134842938548

Al 4.782133513269347

Al -4.721930908181282

Al 1.564520922037506

Al 4.147662996982632

Al $\quad-3.090258256848147$

Al -2.744720693557891

Al -0.463083817784925

Al 2.256638010283908

Al -1.110055555830222

Al -0.793090600783486

Al $\quad 1.535793752348145$

Al 4.206792604358883

Al $\quad-5.138617086001998$

Al 0.985046765045894

Al 1.164063323391998

Al 3.609079707041031

Al $\quad-3.175800408276068$

Al $\quad-3.451459492047508$

Al 2.986146629892723

Al -1.853902316147620

Al $\quad-1.415687949540178$

Al 0.918789363419947

Al $\quad-1.213844892084301$

Al 0.293136700653736

Al 2.945297264007591

Al 0.621224972546573

Al $\quad-3.847257521224689$

Al 2.471302589824484

Al -2.103130207350411

Al -1.900835894720746

Al $\quad-0.403870058915967$

Al $\quad-0.081020425130353$

Al 1.682950840529390
1.810312376512039

3.647808092758849

1.031213852987015

2.870497356747606

$-0.711736174983121$

$-1.580037721985109$

0.267285249815760

0.143051645971447

0.364160404675776

$-3.295429432726158$

$-2.387179477924892$

2.532815319042284

$-1.429426517596605$

3.354441850922829

4.064881628826795

0.721006201273347

$-3.175901592332344$

1.449812753015776

2.319851857758865

$-1.390578725782760$

$-1.154079999658503$

$-4.896774136279277$

$-0.287403473543240$

$-3.199540522927824$

0.842414500460107

$-2.899541935510702$

3.022216503965060

$-1.033548495245503$

3.656437234434717

$-4.904923062798523$

1.037876235892941

1.843707169816497

$-2.804224568666316$

$-1.071735708608673$

$-0.848481677779866$

1.114935083328156

$-2.823780920682037$

3.190633258576060

$-0.703481915488146$

1.312447483737708
$-3.168246320466817$

$-3.792136898090773$

$-3.129275414999467$

$-3.771992945851408$

$-2.409678975419629$

$-2.625829011071579$

$-3.245631772373326$

$-2.344149393315284$

$-1.746305031879164$

$-1.783924024278667$

$-1.609601325211175$

$-1.418190766295842$

$-1.145673934387824$

$-1.302651942334609$

$-1.393600563058298$

$-0.743335116355055$

$-0.432537326691126$

$-0.653165280768849$

$-0.674350701610534$

0.278894132827505

0.027712622771583

0.406485425266318

0.198394409222885

0.862721837398196

0.726933087676903

0.810232869711390

1.008243301117307

1.345028509284012

1.051732952673307

1.706442359425619

1.719544184712245

1.802045600832436

2.220061674376410

2.748350831167015

2.598295463101714

3.106228479958876

3.455850107680314

3.350729190566126

3.845177053344639

4.121172651344658 


\subsection{Al_41}

41

\begin{tabular}{|c|c|c|}
\hline-1.058794427570684 & -1.020294426625927 & -3.278050712099232 \\
\hline-0.329113028953811 & 1.707779064209960 & -3.895370954607547 \\
\hline-0.542724141473277 & 4.321538346879098 & -2.959395666017612 \\
\hline 0.908117579863818 & -2.536008625950129 & -2.050497259517187 \\
\hline-1.615826124189292 & -3.549794721751199 & -2.434693644984424 \\
\hline-2.815988811896961 & 0.780003678233296 & -4.298515249502582 \\
\hline 0.336491321494302 & -5.151320557074119 & -1.342865064704416 \\
\hline 1.540750664617224 & -0.001180977683276 & -2.892514479966295 \\
\hline 1.320730921098804 & 2.620301785326880 & 3695735 \\
\hline 1.060103709168301 & 5.290480394263469 & -1.0244 \\
\hline-3.288799328472281 & -0.165997718093312 & -1.74903 \\
\hline 2.848251463628957 & -4.158428527400914 & -0.927492649944513 \\
\hline-0.733062385997469 & 0.891746089087544 & -1.242971 \\
\hline-0.996138932161289 & 3.51084974385 & -0.3311 \\
\hline-1.742659170182439 & -4.44038407223 & 3814010 \\
\hline 3.460252649116379 & -1.5 & -1.6675 \\
\hline-3.778131373289575 & -2.732471848253920 & -0.9527 \\
\hline 74572 & 1.0 & -0.9 \\
\hline-1.229526155705624 & -1.672098424152386 & -0.4239 \\
\hline 4.7978884 & 2.0 & 977 \\
\hline-3.064015920028441 & 1.842912707175449 & 66281022 \\
\hline 3.312155067776571 & -2.40806023274 & 1361966 \\
\hline 0.927335586661608 & 1.793980055039427 & 175852 \\
\hline-0.998736065707625 & 0.0885328485 & \\
\hline 5.089757327372745 & -0.530696744520679 & 9987210 \\
\hline 2.953028074746543 & 3.672526178196408 & -0.032305022084669 \\
\hline-3.505669 & -0.75557752906 & 7081906 \\
\hline 1.281109881406254 & -0.772952263050302 & 0.006027 \\
\hline-3.846234170062171 & -3.416507150985084 & 1.702764973001084 \\
\hline 2.601456556713062 & 2.853951595434005 & 2.686286171607009 \\
\hline 0.709276925720511 & 4.561325093276182 & 1.620301103386057 \\
\hline-1.227537260759284 & 2.805108476546261 & 2.337052619246514 \\
\hline 0.792647625673425 & -3.376646732556365 & 8400292 \\
\hline-3.224035869845046 & 1.151989406816838 & 2.823451848166032 \\
\hline 2.943157075391843 & 0.220757107210494 & 1.943406211126810 \\
\hline-2.605309735694560 & 2.590239913781335 & -2.316016358010636 \\
\hline 0.760669287764600 & 1.098431136392412 & 3.499229945914575 \\
\hline-3.399437868588243 & -1.435411822474325 & 3.635757871817574 \\
\hline-1.257488938950106 & -2.571614540496094 & 2.252497671271158 \\
\hline-0.828430198898220 & -0.863899551591164 & 4.261736456295989 \\
\hline 1.303158355508156 & -1.704179434491326 & 2.866669929818761 \\
\hline
\end{tabular}




\subsection{Al_42}

42

Al $\quad 1.288398893562944$

Al 1.921561393695340

Al -1.220129979512958

Al -0.579439678219911

Al 4.445711012291271

Al $\quad-0.005582929719898$

Al 3.024816527311433

Al -3.082409482521229

Al 2.520198725281015

Al -2.465457049919564

Al $\quad 0.514058338385730$

Al -1.954721856884078

Al 1.097441749451017

Al 5.091973001113722

Al $\quad-5.030798007684562$

Al -2.074505318970127

Al 0.648787713190135

Al -4.431978414591816

Al 3.659633414121885

Al -1.432471845258521

Al 3.203771347021972

Al $\quad-0.907587623044535$

Al 2.318206141683810

Al $\quad-4.085348431419762$

Al $\quad-1.258875348606125$

Al 1.650430014257651

Al -0.313377492949801

Al $\quad-3.416799048879047$

Al 1.305478413251910

Al -2.855360489151562

Al 4.320524782040005

Al 0.176600290025233

Al -2.972735582482513

Al -0.271683464517050

Al -2.422899195503280

Al -1.916168093847169

Al 2.855576476692358

Al -1.185002875834508

Al 1.370332210810739

Al 2.364267456134833

Al -0.777045881847418

Al 0.882610191042394
2.592648670819079

$-0.040359743926663$

3.575548706198660

0.874152657325247

$-0.138153628398884$

$-1.770615368838017$

2.128406590135910

1.880091802110142

$-1.851818505198406$

$-0.743750263883555$

3.079337792456862

$-3.363091678335216$

0.433124214804124

$-1.878953987355663$

0.303757325623410

4.094069453585281

$-3.461710470287516$

$-2.314346791374370$

0.400976442137823

1.357885173657127

$-3.579664903868397$

$-1.292907784071296$

2.677676013518825

2.367597534039311

$-5.029664842255768$

$-1.310996963332983$

3.498603575081624

$-0.301099764234266$

$-5.134302793088906$

$-2.919841596087765$

$-1.453291744294749$

0.867231138734331

4.422464822431429

$-2.979257972856122$

1.685518733085278

$-0.965633787698920$

0.735811467541419

3.687827602871097

2.913086652119761

$-3.139972118613164$

1.061735205529837

$-0.968116865805985$
$-4.198846286137164$

$-3.729622616447640$

$-3.688759688511284$

$-3.159723713706938$

$-2.707528336794345$

$-2.738859866271747$

$-2.097460909440416$

$-2.636972264195935$

$-1.769736133745569$

$-2.108727295661118$

$-1.563940060048909$

$-1.575474210219395$

$-1.051985172655803$

$-0.709927178026026$

$-1.558360329180930$

$-1.110524523814439$

$-0.731844426081318$

$-1.013017993672388$

$-0.044940098254390$

$-0.478601368610677$

0.236693299917992

0.064464127716933

0.497768595785763

0.014540424338115

0.463165048148229

0.938691221696459

0.956165949929110

0.630784662938435

1.391074440341526

1.178524095694257

1.920485698405649

1.658108848815003

1.478040326741281

2.144701370487272

2.135864552902781

2.861272914638096

2.615036401100163

3.566384095877780

2.999625621401467

3.004669017223137

4.200795412060705

3.717996345316200 


\subsection{Al_43}

43

Al $\quad-1.610274542508295$

Al -3.496337682687113

Al 0.379858867374196

Al 2.443833264379908

Al 0.401254384796006

Al 0.405431091729627

Al 3.077866636194173

Al -1.558573394912305

Al -1.525585389754539

Al $\quad 0.543353475566871$

Al 1.171620397955174

Al $\quad-3.329531307446007$

Al 3.902299579131876

Al 2.616402566996076

Al 3.220816600824975

Al -1.392952845009196

Al $\quad-5.231384514395317$

Al 0.551476985366115

Al $\quad-3.402190680789429$

Al -1.465360688798560

Al -3.243678352004157

Al $\quad-0.601309795057789$

Al -1.343481691809181

Al -5.104870715964935

Al 2.116005496489826

Al 1.305448294750354

Al 0.640236402568542

Al 4.085337965378365

Al 3.297871245628134

Al 2.606809402834086

Al $\quad-3.250946158260596$

Al -1.410744690445231

Al $\quad 0.554094178230518$

Al -2.338435317661048

Al $\quad-3.141930932533098$

Al $\quad 0.285047850835076$

Al -0.498882858840526

Al -1.293522851034648

Al 2.166410382556247

Al 1.369227259611019

Al 0.676053875787440

Al $\quad 4.096110584091898$

Al $\quad 3.327127620835418$
0.048282533709465

1.874079670315009

0.415105119036982

0.214033487340355

$-1.878406459132842$

2.108611555494415

$-2.480556112583783$

3.854585307481337

$-2.396163158857656$

$-0.286532185486449$

$-2.982110597143964$

$-0.470032238318462$

$-3.627432665507710$

1.843097137384067

$-0.883000416448988$

1.500191517454422

1.411904521011367

3.502199758619993

3.373650453625723

5.265360697272451

$-2.768362514478348$

$-3.412384912371658$

$-0.772781759744509$

$-0.851621068085597$

$-4.172064213373657$

$-1.389544966483658$

1.258244025497179

$-2.154051689343354$

0.580056823319248

3.221459524243874

1.134293543802153

3.017661360771720

4.941493275442040

$-3.821582470661784$

$-1.187003167518858$

$-4.545538728513559$

$-1.840307318230899$

0.799947730872615

$-2.577404370855043$

0.072772098977278

2.721710782624809

$-0.641732943910558$

1.979873032754897
$-3.447237721096299$

$-2.827385487700468$

$-5.300625317200580$

$-3.596316254148393$

$-3.907385188624088$

$-3.141940894256632$

$-3.516199079633680$

$-2.535927600398002$

$-2.044106695124850$

$-1.648487597172295$

$-1.576277478786158$

$-1.367476126561240$

$-1.202978762391286$

$-1.412134550124435$

$-1.263064763399512$

$-0.944764138743070$

$-0.734290292424326$

$-0.779275958688482$

$-0.331328811538976$

$-0.167118307834446$

0.116365563825722

0.367872255799773

0.522730573683853

0.770205054119348

0.761947522526990

0.765894739178009

0.823665545703008

1.064747218545124

0.964456378797061

1.007323490617063

1.220809188877871

1.499228728022311

1.628228257175570

2.510396171369369

2.650441142580094

2.754041927212606

2.936223953642076

3.120349027531798

3.094636443742298

3.220360904974093

3.230000796476551

3.339541592473241

3.374854548973277 


\subsection{Al_44}

44

Al $\quad 0.979638404963167$

Al -1.754260860955373

Al 3.733372186249419

Al -2.471620217294497

Al 2.786919582135075

Al 0.145153931116425

Al -0.621697642667852

Al -0.605257537018469

Al $\quad-4.474955428541820$

Al 2.099876041885077

Al $\quad-3.356235365624672$

Al 2.011706334703689

Al -2.431392305684351

Al 0.517141603562122

Al 4.018794164810121

Al 1.292686491553924

Al -1.439156214121768

Al -4.172779494012408

Al 3.484079099187143

Al -2.470916644937628

Al -0.901874573595489

Al 0.587835871684810

Al 3.038362217419168

Al -1.235851377701582

Al -0.446789940557279

Al 2.582412080478145

Al -3.487223594565118

Al 3.076029174606399

Al -2.539644511228778

Al 0.957124992832727

Al $\quad-2.714300135392881$

Al 0.217743728712572

Al -0.982742236823697

Al 1.218330874725423

Al 2.458981361403881

Al -1.771699664099536

Al 1.468600539454684

Al $\quad-1.091197324849947$

Al $\quad 0.967962315943080$

Al 2.090829423007706

Al -1.141421261922217

Al -0.482312325289444

Al 0.122391027282278
0.778785655607951

0.148098127596057

1.358963324046426 $-2.224787538299033$

$-1.013114322151681$

$-1.519850507329439$

1.991334714188998

$-4.120032471506920$

$-0.532855009747417$

2.642074040563786

1.383569084699390

$-3.516496221009179$

3.492776067133626

4.172474434443423

0.894512077434985

0.306690248917892

$-0.323644227785813$

$-0.995331726050313$

$-1.698090162631997$

$-3.072888957873801$

$-5.119141947071699$

$-2.680800622845537$

$-4.210404644530770$

5.450803069666136

2.058934655936968

2.768369311767884

1.367988038541450

0.243770023209148

3.524422892347596

4.330735664201834

$-1.091759624741155$

$-0.561341600360857$

$-3.103703029546143$

$-4.790254569011045$

$-2.309660354826573$

1.033569265505649

1.780376763944034

5.609922740448699

$-3.094268995196819$

$-0.598307261093170$

$-1.344210120810159$

3.176237353958197

0.867136925637174
$-4.159801839175485$

$-3.972202482991848$

$-4.282955124860052$

$-2.946659646587122$

$-3.311037599651656$

$-2.976227245861579$

$-2.355680185720557$

$-2.489080562354324$

$-3.717169094689660$

$-2.575020842670470$

$-2.198551476580469$

$-2.668722263820335$

$-0.962192170245284$

$-1.165789779716068$

$-1.559496297074288$

$-1.185371168828008$

$-0.996359344789047$

$-0.995043258866808$

$-0.838229223693553$

$-0.427414329458344$

0.002470684087571

$-0.449560988603475$

$-0.268824158334539$

0.306835971073163

0.408303923806638

0.137196880814265

0.555242131906169

1.009874706108228

1.783867222815408

1.542392853645577

1.408878833416292

1.284261263675422

1.764372360447663

1.629041644290606

1.527667519663611

2.629355362362340

2.405788640899692

2.938515627620530

3.691992153448684

3.542281018524806

3.764310025853581

3.627010885737649

4.693475262012111 
Al $\quad 0.737357209167746 \quad-1.460600565377815 \quad 5.848254112362975$ 


\subsection{Al_45}

45

Al $\quad-2.250555778190532$

Al 0.183052194619018

Al 2.587018132303134

Al 0.235045520479462

Al $\quad-2.363581089418928$

Al 1.711041090077662

Al 2.310689174607930

Al $\quad-3.711324830645768$

Al -2.850263573489304

Al 0.898631846565362

Al 3.248006094932155

Al -1.480352810548572

Al 3.930536112533515

Al -1.807794918181379

Al $\quad-0.313396274340509$

Al 1.034120409387905

Al $\quad-3.106392385545186$

Al -1.109207798332744

Al 0.601243795386980

Al $\quad-4.316581886918838$

Al 3.470921365191044

Al 4.234101283618145

Al 1.599152172501046

Al $\quad-0.848494770265864$

Al $\quad-0.549933258737374$

Al 2.246950483879154

Al $\quad-3.848640381213069$

Al -1.802788082115672

Al $\quad 4.864461958916161$

Al $\quad-3.079652626881389$

Al 1.696136029388761

Al $\quad-1.995090962044629$

Al 0.106233819402130

Al 0.429047089690856

Al $\quad-4.148530683257718$

Al $\quad 0.565215748652086$

Al 2.757293688303090

Al 3.261586368267446

Al -0.721994971409773

Al -2.471511852858684

Al -2.008949595192425

Al 2.274793194673684

Al $\quad-0.370291249257539$
$-0.715219243784238$

0.920730435491574

$-0.489623102959847$

$-1.815589557535087$

1.959121418760869

4.208315596362636

1.997703095498029

$-1.353939937318291$

3.032006736627318

$-3.779864722621939$

$-2.505352748561187$

0.581395803009421

0.132342820166173

$-3.190423729204567$

3.258683292382443

$-0.638880324264456$

$-3.653214184975233$

$-1.785506112201188$

1.784838897833675

0.973724922566758

2.430880650617228

$-2.059063099338476$

$-2.842597328199934$

4.310623689525675

$-4.564291141462033$

$-0.136114732876736$

$-1.500625620075068$

0.593106050702071

0.472750412138067

3.207690523004111

3.846524116168109

$-3.266048100085281$

$-1.321907325041771$

1.340630416891901

0.859067611360251

$-4.151641167945160$

$-2.616030440365062$

1.911172640519936

3.925763523843209

$-0.986312495477783$

1.836310307205457

$-0.266245521011792$

$-2.604798420168708$
$-4.080387017295015$ $-4.239303168184971$ $-4.038310943456543$ $-4.302078672386088$ $-3.956204008080005$ $-1.354934071948167$ $-2.886753598405523$

$-1.819433795873218$ $-1.507494688353491$ $-2.547308317806882$ $-2.281219257644935$ $-1.810706173775055$ $-1.733572431520802$ $-2.788620440007318$ $-2.800819264070413$ $-1.810051296070815$ $-0.454727109901576$ $-0.464781850451921$ $-0.576370021920994$ $-0.471280074479075$ $-0.399962907986637$ 0.196394118252348 $-0.068135657907931$ $-0.216464320738769$ $-0.384077446496905$ 0.616625661132721 1.010498150332882 0.843997765263497 0.813523977637436 1.203892340363296 1.342426783439244 1.987646084660250 1.880806667144142 2.005905230205212 2.241928664933194 2.083363256998977 2.403834773324679 2.429821973460175 2.467712540994967 3.326955437496681 3.578442089931864 3.695546239084704 4.141624164796623 
Al $\quad 1.143932002598152 \quad 2.586499234898120 \quad 4.176894030197543$

Al $\quad-0.233879797128854 \quad 0.073406859900853 \quad 4.545156585112538$ 


\subsection{Al_46}

46

Al $\quad-0.210768845157771$

Al 2.313425607740385

Al $\quad-2.356346270195679$

Al $\quad 0.075205860490657$

Al $\quad-2.574375905075281$

Al 2.053251600350890

Al $\quad-0.089309448163688$

Al -0.536715432775196

Al 3.800427088454493

Al $\quad-4.022694614106545$

Al 2.078857411438289

Al 2.080006696202904

Al $\quad-2.568143077406280$

Al -3.010349816113492

Al -1.299874894507134

Al 2.445467301966124

Al 3.825446942946877

Al $\quad 0.571553311417770$

Al $\quad-0.281620378570356$

Al 4.143553348397839

Al 0.835026511581152

Al $\quad-4.468926311781731$

Al 1.405181506338934

Al $\quad-4.205411883152169$

Al $\quad-0.966748646036679$

Al -1.710185401709086

Al -1.471100546920674

Al 2.125265459219852

Al 1.425366620432506

Al $\quad-3.205051788436822$

Al 0.640284487719164

Al 3.225570960835395

Al 3.416018556476400

Al $\quad 5.022491687928758$

Al 0.879308920990225

Al $\quad-2.570954571931389$

Al $\quad-3.999749329798337$

Al 1.567129630451708

Al -2.591343363120085

Al -0.853771157593561

Al $\quad-3.008832241217779$

Al 3.470557282134827

Al -0.201603683014531
$-4.256117333551958$

$-0.127124463825430$

$-0.221835931040829$

1.298268183864307

2.290909597784891

$-2.885914454026133$

$-1.585519668046018$

3.560286107262543

$-1.600790513505604$

0.332594905552524

$-3.709517051377015$

2.568441340299801

$-2.209515750501053$

2.861191027014486

0.455103111006922

0.169951944953850

$-2.256776525356632$

1.836739900989931

$-3.679874835802828$

1.010064540373728

$-1.103234637471141$

1.075639040916485

4.479606887696113

$-1.838480086221330$

4.134686211647363

1.014932125562762

$-1.589391422938503$

$-4.009081486566014$

3.719977208335667

3.238785655032048

0.815481079987142

$-0.593793781767248$

2.834216086132280

0.285537766997187

$-1.747110095496176$

$-4.022028789144343$

$-1.025943332228961$

2.426320037513669

$-3.324527977529081$

3.096633793583452

1.187631920040223

1.949362046303667

$-3.451133746096237$
0.617954167411254

$-3.772936917639191$

$-4.445258110331390$

$-4.211139966180753$

$-3.673940491892535$

$-3.234194768188364$

$-4.098561500881339$

$-2.518748204991903$

$-1.656974598322391$

$-2.202947549726371$

$-0.653500003939319$

$-2.946230837356803$

$-2.658223393164537$

$-1.090112130904837$

$-1.875504263697623$

0.163935184548516

0.955919479795002

$-0.547416890750078$

$-2.116646662717552$

$-2.146591174523815$

$-1.446845098169755$

0.462655024109157

$-0.971924558473614$

$-0.447325211019100$

0.283173147796999

0.861980013950941

0.064502550072556

2.065729923419578

1.627029562494279

1.565841027151674

2.120802244445319

2.954574251813163

$-0.326653143263034$

0.505902257267197

1.344157605548636

$-0.667274083097215$

2.150019934798632

4.047373150452627

1.970184225128163

2.878252259172941

3.347814836994914

2.212462231295913

3.295148453792857 

Al $\quad-1.551927951695307$
$-1.141342938854784$
3.273231341165229
Al $\quad-0.610110375573141 \quad 0.956462273297721$
4.638718215426254
Al $\quad 0.966519140537677 \quad-1.219767970801353$
4.301588471179610 


\subsection{Al_47}

47

Al $\quad 3.620623126506262$

Al 1.046189484953246

Al -1.591578720461746

Al 0.818769383675976

Al -1.611688581411601

Al 3.126024480061442

Al 5.263521346432732

Al 0.775393363084049

Al -1.285409531622332

Al -3.770501172399071

Al 3.100628814110076

Al $\quad-0.054312803727118$

Al 2.554461317735623

Al -2.656434633143631

Al -0.306591717633149

Al 2.160090405562054

Al -1.000471135741781

Al $\quad-5.914942420287647$

Al $\quad-3.421676089769724$

Al $\quad 4.588794649450506$

Al $\quad 1.549118803148970$

Al 5.049926397098121

Al 0.313592018562757

Al $\quad-2.233736095399829$

Al $\quad-4.850074202855096$

Al 2.792078445909894

Al 1.401928756113715

Al $\quad-1.272499953479546$

Al 1.052043013377610

Al $\quad-3.865371240212734$

Al 3.623711895964714

Al $\quad-4.501838681791082$

Al $\quad-2.037896641098964$

Al 4.006256560221424

Al 0.441116544717175

Al -0.863386592123973

Al -3.457202544413455

Al 1.643190992637590

Al 0.120062337411131

Al 2.481154400018399

Al $\quad-2.540586421800000$

Al 2.754291872381256

Al $\quad-3.106963787272701$
3.553611262250820

2.682832457514971

$-0.793661899877870$

$-2.328991987942253$

$-3.466862315194031$

$-1.098054741393824$

0.493706624699836

0.449804078382028

1.452922131747121

0.282318694314096

1.748379314473432

$-0.810218570682261$

0.605132772752702

$-2.178768648198742$

$-3.581192692156244$

$-2.336206732670323$

3.815674914053682

1.482955564561781

2.604304724988395

$-1.201363295753383$

4.691355730188750

1.611762027774825

1.408882949679537

0.205984447552527

$-0.888439136965751$

2.560551495744797

$-0.915006191611676$

$-2.213476389703755$

$-3.679270898371462$

$-3.220557833115215$

$-2.527227097789766$

1.430822919491639

2.545807495557868

0.221604746404417

3.714877901915910

0.107708765384883

$-0.924047454762198$

1.245117196557135

$-2.379434487122207$

$-3.756855205764256$

$-3.317782074915248$

$-1.101392417986293$

1.329736522123758
$-1.941561348898237$ $-2.514604698508089$ $-4.145354153233464$ $-3.814702748361261$ $-3.950985405854373$ $-3.538022424087788$ $-3.011411580664004$ $-4.169624288768762$ $-2.590809594337205$ $-2.698028405497102$ $-3.971506371527921$ $-1.689439745715369$ $-1.446230682724391$ $-1.774644196095529$ $-1.543268055233378$ $-1.339181579179435$ $-0.936186927433230$ $-1.424344770527856$ $-1.169552508725777$ $-0.998890388884485$ $-0.701846460152920$ $-0.586528439987896$ $-0.122393696442664$ $-0.291454693235208$ $-0.444594671171245$ 0.516917761521869 0.842800231716275 0.642084179094653 0.815990930263739 0.558941482861364 1.056975630391012 1.038477535582917 1.279636762467003 1.441237312316840 1.566974224054171 2.089043344538254 1.953515593473215 2.494149623091133 3.048196217564010 3.155261607401071 2.859168966562477 3.399007353216231 3.460375685674832 

Al $\quad-0.615365832470395$
2.403527946893654
3.702087274119979
Al -2.083978566819741
$-1.088608773427511$
4.357469216407797
Al 0.446389151068480
$-0.020454592618856$
4.622437504091748
Al -1.686850194267889
1.178490753014568
5.914419398837010 


\subsection{Al_48}

48

Al $\quad 0.301899832630578$

Al $\quad-2.094437008155129$

Al -0.578651490473435

Al $\quad-3.863445483974847$

Al 2.491633976449856

Al $\quad-2.793877927716262$

Al $\quad-0.209412358092742$

Al 1.307754661438928

Al -0.058514519092414

Al -1.249715104313260

Al 4.731568536093752

Al 3.193107352378883

Al $\quad-2.678956964526054$

Al 2.049572158693778

Al 1.684463525324643

Al -2.529996900672481

Al $\quad 0.562048759480209$

Al $\quad-3.486776561325799$

Al 5.221846460310607

Al 0.042626626076498

Al $\quad-0.523159214542098$

Al $\quad-4.573248068197347$

Al -0.946455623504871

Al $\quad 4.046372687018346$

Al $\quad-2.021043783987814$

Al 3.854791809470623

Al 2.626132722580813

Al $\quad-3.152016962793120$

$\mathrm{Al} \quad 1.566539512873112$

Al 2.203676401580720

Al $\quad-2.198604064565086$

Al $\quad 1.034208192610960$

Al $\quad-3.247092358620990$

Al -0.024872370322786

Al $\quad-4.224386914382967$

Al $\quad-0.966779366295901$

Al 4.485030876094704

Al -0.611063682833608

Al $\quad-5.172435292173324$

Al 3.348638452618717

Al -1.612357975841414

Al 2.969992661698914

Al $\quad-2.637476771964597$
$-0.694184775728370$

$-1.829088175388051$

1.863611833032760

$-0.345717386334329$

$-1.379824357375840$

2.279973626571991

3.306541080867484

1.081818524075352

$-2.294213478183410$

0.173960879043564

$-2.129742776658348$

0.192290758473327

$-2.740341469893638$

$-2.751967442391063$

2.399983768561237

3.801616575824474

$-0.402244328119609$

1.253884561316120

$-0.456995965630879$

4.549808200715621

$-3.129060404219906$

$-1.262194871944065$

1.852820832879008

$-3.423361456755083$

$-0.793821542314726$

1.788988795099138

$-1.113571505364106$

$-3.397487695882241$

$-4.115946648913416$

3.808023671014853

5.279523447081830

1.191359299905240

2.811191150837731

$-1.479007375481856$

0.359296695621262

$-4.094223854084825$

$-0.268498804273571$

3.390853616303119

$-2.147386263777646$

$-2.829905494267975$

0.809924163494934

1.870479778093440

$-1.716679980874742$
$-5.360218486047732$

$-4.588863826173792$

$-4.956402485978772$

$-3.218208415916992$

$-3.754173558960298$

$-3.480492268388654$

$-2.690865602889600$

$-3.270708134928171$

$-2.969105961469340$

$-2.819161576546527$

$-2.225111204823458$

$-1.604775778282923$

$-2.125593985396371$

$-1.408302222027313$

$-0.951845241095977$

$-1.268461103119257$

$-0.897452368873646$

$-1.024200993796777$

$-0.073152704427452$

$-0.356988982165928$

$-0.490703075769344$

$-0.758117500462946$

$-0.238544984430760$

0.160615273687521

0.022414908429804

0.582056160159288

0.738037243724600

0.429274297325854

0.986588533209769

1.333863158221279

1.005733535885360

1.461860731249324

1.258869967677303

1.695572799301985

1.514279576221352

1.991607416429266

2.504939413349883

2.209122679042956

1.768447113465744

2.711341647021396

2.398059213454331

3.226638602006483

2.705715112075650 


$\begin{array}{lccc}\text { Al } & 1.876477262380041 & -0.601652960277930 & 3.424360930175862 \\ \text { Al } & 1.402773291642795 & 3.980094490172380 & 3.915230958905374 \\ \text { Al } & 0.823679492305212 & -3.138597980018146 & 3.747911070820013 \\ \text { Al } & 0.325284374553394 & 1.506506543017700 & 4.235149608304999 \\ \text { Al } & -0.695342857937764 & -1.016835297848745 & 4.503760511826590\end{array}$




\subsection{Al_49}

49

Al $\quad 1.292534131588038 \quad-4.569740489149821$

Al $\quad-2.166279724438157 \quad-1.543489843898820$

Al 0.353998472683346

Al 1.370263010346392

Al -1.399603722304636

Al -3.986971450442901

Al -0.462863868995134

Al -3.216982647474322

Al 0.018051981884323

Al -2.665463794261791

Al 2.471966207477228

Al 3.161384461383356

Al $\quad-4.601172679379957$

Al 0.548013330673527

Al -1.948740497098017

Al 2.353969217435742

Al 0.623154251073515

Al 4.287055995572041

Al -4.006543237938067

Al -1.324915441708729

Al -2.084876668533745

Al 1.885166489449650

Al -0.598745046795051

Al -0.048727604778408

Al $\quad-2.590183356662320$

Al 3.653672128605587

Al 2.325994649704779

Al 3.971599398227005

Al 2.152881589500108

Al 1.253733990539743

Al 4.855097982667038

Al -4.738296528245702

Al -2.143281684263579

Al 6.095196555065225

Al -3.299397258078974

Al -0.647090470655732

Al $\quad-0.753530715800236$

Al 1.782564913104677

Al 0.270525625239840

Al 4.125469673551649

Al 2.952398397913873

Al 1.010966402895674

Al -2.931113776140881
$-0.669409049146827$

1.540294845437771

1.035644975544361

0.053047784669436

3.280650305142974

2.495144996888477

$-2.747718620606441$

$-3.466865308804201$

$-1.398875422706848$

0.781834352486413

$-1.891164707506983$

$-0.202497934125130$

$-0.935680991201076$

3.580980005495029

4.928846148882592

$-1.736641090853965$

0.682656998803376

1.582295422360719

4.441735060988133

$-3.045573051921080$ $-4.272035169504821$

$-1.861811485352460$

$-2.962655379477723$

$-3.278497935433442$

$-0.720421377104129$

2.936598211595665

4.199895274922744

2.105652236392995

0.440498793433767

$-1.403359155295274$

$-0.288418764850933$

$-2.020466437672194$

2.958614220748254

3.839283584977412

$-3.799876727663764$

$-2.540740166348531$

0.698538480808415

$-1.059730513785333$

1.517755089572473

2.870058437283490

$-2.429386886197893$
0.858005198332940

$-4.288139326829584$

$-4.829739136312480$

$-3.875861688184359$

$-3.667316847197740$

$-3.101291708208019$

$-2.781415953413322$

$-2.255838675107464$

$-3.097381558807429$

$-2.450644540408364$

$-3.417428258123216$

$-1.932869430733758$

$-1.269457314552088$

$-1.817265687953929$

$-1.448090471083948$

$-2.451830716031960$

$-0.846441708092842$

$-1.440173010988117$

$-0.361700275531998$

$-0.479257845875814$

$-0.885259839995879$

$-1.315008775674649$

$-0.924311066544401$

0.458042831004223

0.420434533514969

0.771943542734421

0.328519679825394

$-0.332875487246868$

1.273574602520812

$-0.385373339611368$

0.181696351875615

1.375475917451775

1.457714284769944

0.694529205065809

0.894920521991448

1.361922296560275

2.333483726800951

2.620737786185355

1.768926678251383

2.418047397930312

1.835352053274525

3.321413382506293

3.188530847531911 


$\begin{array}{lrrr}\text { Al } & -2.625938990236324 & -0.010706313150841 & 4.330543096068372 \\ \text { Al } & -1.820124502559329 & 2.326760903260793 & 3.096175052501172 \\ \text { Al } & -0.349043478199157 & -1.433800214350192 & 3.606088336976267 \\ \text { Al } & 2.047580863349786 & -0.076102866695168 & 3.793920457067172 \\ \text { Al } & -4.316138302600486 & 1.065773622296375 & 2.489356909759753 \\ \text { Al } & -0.137214272340529 & 1.003106150812231 & 4.775617972008520\end{array}$




\subsection{Al_50}

50

Al $\quad-0.082844433460918$

Al $\quad-0.026507432458194$

Al 1.869834107182733

Al -2.055853622834928

Al -0.174009695008172

Al 0.111138096936301

Al $\quad-2.259372540405078$

Al 2.114663021712163

Al 3.970818150271093

Al -4.087197874723653

Al $\quad-0.081295355220712$

Al 1.879297555740775

Al $\quad-2.261491221367962$

Al -0.224733469704656

Al 6.317002397396328

Al 4.410401244329744

Al -4.219057907808481

Al 2.499961494503935

Al 0.453799371751359

Al 2.389327874543662

Al 4.314080620640731

Al -1.954491946376764

Al $\quad-1.965249662521327$

Al 0.205805954499132

Al $\quad-4.234540446612972$

Al 0.004918099060465

Al 2.117447176799850

Al $\quad-2.399762163369214$

Al $\quad-0.168071399309087$

Al $\quad 4.818297036686884$

Al 3.059982797671466

Al $\quad-3.730796687705934$

Al 3.113092879708471

Al 0.952153227144354

Al $\quad-3.819990311047840$

Al 1.124013460483939

Al $\quad-4.036547171263448$

Al -1.868244046104213

Al -1.549713793674961

Al 3.461328780517144

Al -1.484954984812852

Al 1.458732888318799

Al -3.134807628279924
$-0.155634593848422$

1.876017854006001

$-0.180601760263160$

$-0.044972343971271$

$-2.055787210895982$

3.499686778520575

2.146369864734554

1.701339447974345

$-0.273697175395302$

0.075879457553063

0.045148311929676

$-2.013677662635965$

$-1.962225271530802$

$-3.788465808982154$

$-0.329222621622254$

1.725216624936202

1.840556642147142

3.611965047233388

1.768540216386315

$-0.226203499323971$

$-2.209043944007666$

3.323306179579049

0.218059930307248

4.875210255054606

$-1.451339739262597$

$-1.802938396892698$

$-3.669567807224671$

$-3.452648745068608$

$-4.878336374290167$

$-0.168473725930774$

1.897039883844302

3.041166370867035

$-2.213899128596584$

$-0.139972807633670$

0.245018113070490

3.552415554900815

$-2.632129256421955$

$-4.226113847410716$

$-1.357571144695052$

$-0.028142944207245$

4.485169419139368

$-1.624069772569163$

1.602983335924737
$-6.429235475905539$

$-4.522909667643668$

$-4.425082839921967$

$-4.509123610318191$

$-4.343415350002210$

$-2.407331104858631$

$-2.861155935517250$

$-2.456459471322296$

$-2.695227539999180$

$-2.721590470637221$

$-2.428964402977744$

$-2.413774159519313$

$-2.550597937577418$

$-2.267975366234273$

$-1.168380922823021$

$-0.823772908659905$

$-0.763135030922324$

$-0.508216319620789$

$-0.210168920682394$

$-0.384202635834299$

$-0.703033122429449$

$-0.565157740388040$

$-0.591576487300516$

$-0.174922300654581$

$-0.612927070026002$

$-0.349408389826275$

$-0.303742855195338$

$-0.443741629197913$

0.135639634289568

1.140201356379350

1.569038035353822

1.608167802458341

1.826108289898807

1.825624396053680

1.487136873446230

2.001565940116627

1.783267852081911

2.161562878142616

1.719080539729820

3.479913356979997

1.926235583095274

4.123954109882439

3.865107074478580 


$\begin{array}{lrrr}\text { Al } & -0.782491494430040 & 3.005126749550653 & 4.094061017669031 \\ \text { Al } & -1.288600719706166 & 1.578605020033597 & 1.743978055512841 \\ \text { Al } & 1.526301987359375 & 1.661772069677468 & 3.915991168774630 \\ \text { Al } & -3.264201404045243 & -1.099620583511919 & 3.932561641214265 \\ \text { Al } & 0.732145574027708 & -3.227592781773637 & 2.059398525922477 \\ \text { Al } & -1.032555545113265 & -2.673573985836143 & 4.193056446730171 \\ \text { Al } & -0.717160839920327 & 0.108929806431721 & 4.043579087785263\end{array}$




\subsection{Al_51}

51

Al $\quad-0.142616012793992$

Al 0.567400145539004

Al 1.050585693991319

Al $\quad 2.226787158020080$

Al -2.136253590019738

Al -1.330981336772554

Al -1.370208412131422

Al 1.096466612306532

Al 3.326389001675565

Al $\quad-0.173811168353467$

Al $\quad-4.118727056686962$

Al 2.600720947281772

Al -0.002043570805501

Al -3.561733512464423

Al 4.101177980199044

Al 1.026490763583858

Al $\quad-2.532167798669901$

Al 2.129132016855952

Al 1.621922117081112

Al $\quad-0.777291812056447$

Al 3.052887495653767

Al -3.235189227644581

Al 0.581186915482121

Al $\quad-5.868385993338515$

Al 4.664409705886039

Al $\quad-2.219010649178038$

Al 1.981245631204173

Al $\quad-4.973563313014687$

Al -1.387150167865132

Al 3.287849125633715

Al -4.349180909403045

Al $\quad-0.315176255526460$

Al 1.168582283546597

Al -1.423083274908080

Al 4.308131888583599

Al 2.949575852421070

Al $\quad-0.414562914654468$

Al $\quad-4.011356985079884$

Al 2.069995961448821

Al 0.781529365625149

Al $\quad-3.209889761157984$

Al -2.397656836931478

Al 3.288384107518617
0.574094847716967

$-1.842765983232809$

2.858355506529918

0.422898198423891

$-0.560974883399636$

1.984921671240009

$-2.733547932754288$

$-4.006978602790666$

2.876434346571797

4.444847319795910

$-1.610840964729542$

$-1.736717912057628$

$-0.292135716157929$

1.122272504284471

0.517490947494336

2.054443517552164

3.667995618439372

4.625339516680237

$-6.215626375631315$

$-4.789862412521345$

$-3.885347320816062$

$-3.628329991583255$

$-2.388246500507395$

$-2.705596880211009$

$-1.743190025659496$

$-1.039632638855004$

$-0.191337507760879$

$-0.133866117017014$

1.443573996687300

2.270452353661412

2.457117516090308

3.975460071820814

$-4.570368561007476$

$-3.122143936530410$

0.029867591120551

$-2.435672279738565$

$-0.549658461375284$

$-2.088966680988502$

4.207913161285397

1.762214249634207

0.516703262786407

3.136343127123443

2.031364419379390
$-5.059622238432010$ $-4.115068606654530$ $-4.165763535445381$ $-3.630860097106033$ $-3.736044699492635$ $-3.043634481473505$ $-2.372978221586701$ $-2.557025366225426$ $-2.642579855662493$ $-2.360007722371531$ $-2.288061319547653$ $-2.256313389255649$ $-1.922456318071289$ $-2.045894857908905$ $-1.638881479395758$ $-1.305202631941285$ $-1.257789358476996$ $-0.885242646379488$ $-0.832084541238711$ $-0.650516100150613$ $-0.614749268442058$ $-0.538931232750690$ $-0.274505240830106$ $-0.384711390879882$ $-0.422564148005318$ $-0.377432932128000$ 0.087852433805637 $-0.067024406027210$ $-0.119068444398410$ 0.261081316008822 0.291602304161284 0.401332402462955 1.361914473322178 1.445989000144646 1.545527444769897 1.712615008937991 1.625733837177155 1.648209091749324 1.795315582710911 1.635120681886267 1.927792540543887 1.905697929798698 3.021850723964256 


$\begin{array}{lrrr}\text { Al } & -0.239330019619912 & 3.734351978589604 & 3.311187963123885 \\ \text { Al } & 2.028241074681356 & -0.407931850147028 & 3.049801715439978 \\ \text { Al } & 0.594262635163217 & -2.710596772949779 & 3.181861795971324 \\ \text { Al } & -2.172303756564954 & -1.469218097522228 & 3.540305372877221 \\ \text { Al } & -1.445290967579915 & 1.216160751237007 & 3.673636809061264 \\ \text { Al } & 2.203860704878890 & 4.115814009146233 & 4.599400840338486 \\ \text { Al } & 1.071340373215357 & 1.623413920111268 & 4.614444523044114 \\ \text { Al } & 0.028409745744874 & -0.810289997457886 & 4.926740738978033\end{array}$




\subsection{Al_52}

52

Al $\quad 0.069398330593453$

Al -1.868934680015398

Al 1.960967448860835

Al 0.561732901722699

Al -0.458355522856357

Al $\quad-3.991739043822356$

Al 3.953859956155179

Al -2.339759918586731

Al -1.602424810196066

Al 1.528521550574258

Al 2.657796282030533

Al 0.185119916317047

Al 1.014016450577854

Al -0.970403243787500

Al $\quad 6.176259695646836$

Al $\quad-3.575664816289660$

Al -4.221773262963421

Al 3.802119003434589

Al 4.746627064156847

Al 1.341835352720805

Al -1.615259071521141

Al 2.305607054707654

Al -0.983185141596959

Al $\quad-2.955995274997784$

Al 3.350712132726295

Al 1.039498560868953

Al $\quad-0.304434836760176$

Al 1.433182146830822

Al -1.111328176632703

Al 4.751644793581097

Al -3.536997339957281

Al -2.834662557354036

Al 2.599774718446488

Al -4.443904478800501

Al 3.275604461327717

Al $\quad-0.715453579223338$

Al $\quad-1.775694787296302$

Al 1.959762857584598

Al 0.880452002870877

Al -0.277315437332133

Al $\quad-2.605783318096574$

Al 0.046994543338759

Al 3.670656429570505
0.272818317057853

$-0.289152270899992$

0.702375224685785

$-1.740324345792839$

2.077978317874550

$-0.841373706512696$

1.229842287070337

1.355130746981562

$-2.433223410538321$

2.574827890727144

$-1.207242142526542$

$-0.029674817882865$

$-3.327437234370407$

3.762329986032391

1.766710857660390

$-2.562039940855414$

0.432501456976251

3.166107394140420

$-0.677170014343140$

4.097023155175437

$-0.693196932099948$

0.843504807349614

$-3.503600130505667$

2.919956998773772

$-2.984432787554295$

$-1.552910469711692$

1.793221085888378

$-4.918417111507217$

5.022621493831432

1.230820582630157

$-1.085909033172314$

$-3.763192705253909$

2.760211091117615

1.738616143206283

$-1.049711860313547$

$-1.819600034185905$

1.005771715997501

$-3.246492642652202$

0.453459608689695

$-4.779425049585509$

3.837864405218143

3.197441789011279

0.736433072258810
$-6.518979328472231$

$-4.670400059040214$

$-4.509528661778723$

$-4.649857550115422$

$-4.385358653005807$

$-3.137768832750380$

$-2.744524090963880$

$-2.543048927987261$

$-3.015938541595722$

$-2.523968052829421$

$-2.610652819614398$

$-2.508085962143586$

$-2.566636935164400$

$-2.323747620418764$

$-1.140634492075339$

$-1.063882991580519$

$-0.820087290102636$

$-0.701256109049940$

$-0.874508290318229$

$-0.322337128211549$

$-0.719107006180607$

$-0.546651890203021$

$-0.738435709659418$

$-0.571252996693175$

$-0.597018817570094$

$-0.348356212357929$

$-0.552707978649879$

$-0.483473103475793$

0.089101601841330

1.134019748217973

1.283380686251682

1.251646779701950

1.793696244822746

1.484013232371693

1.395050853938749

1.583632305093662

1.471970834862836

1.794334584403009

1.620857376398174

1.569540261000794

1.966388177029883

1.886590198620597

3.531287921253842 


$\begin{array}{lrrr}\text { Al } & -2.578197355398043 & -2.268578783170662 & 3.588757790496194 \\ \text { Al } & -3.336530804440145 & 0.336690938522151 & 3.665274692652812 \\ \text { Al } & -4.234438735669542 & 2.961708801301642 & 3.983598097903920 \\ \text { Al } & 1.865984273716942 & -1.202354550967820 & 3.708408602434583 \\ \text { Al } & -0.650074686001908 & -0.281371203864863 & 3.851040444038231 \\ \text { Al } & 0.080316466422648 & -3.164090648950358 & 3.866679005598407 \\ \text { Al } & -2.010909926631186 & -5.001819234564740 & 3.675911668328705 \\ \text { Al } & 1.195044512128543 & 1.784356185207891 & 3.984087503419785 \\ \text { Al } & -1.454268100685551 & 2.362416708396418 & 4.008937441326751\end{array}$




\subsection{Al_53}

53

Al $\quad-0.993331550774563$

Al 1.262403569022364

Al -3.012884619750364

Al 3.658488913371389

Al -0.961918711568323

Al 1.195458525058037

Al 3.519299997334505

Al 1.476972490958955

Al 3.659815351181125

Al $\quad-2.755559116088355$

Al $\quad-0.731324655726473$

Al 3.983872679211911

$\mathrm{Al} \quad 1.651239487177955$

Al $\quad-4.636667797078345$

Al -0.800736176358701

Al -2.853349982520220

Al 1.369615886824230

Al $\quad-0.382718728280652$

Al $\quad 3.769227414842875$

Al 1.699056572876058

Al $\quad-0.259487954035652$

Al 3.940897332834666

Al $\quad-2.505790133879720$

Al 1.956946068316752

Al $\quad-4.510804243694994$

Al 4.078145224375678

Al -6.450731191771673

Al -2.576044144857885

Al -0.677860846362845

Al $\quad-4.486395201900579$

Al 1.569532122891244

Al -0.178159412706703

Al -2.184875523311915

Al 1.785861672281019

Al 3.955317989362866

Al $\quad-0.026124662435084$

Al 1.994806365215320

Al $\quad-0.592934062577887$

Al -2.625521018637788

Al 3.970041093440313

Al 1.785929976377600

Al -2.716563560368378

Al -0.425283153953654
0.816206676753513

2.145994378500854

$-0.841945740558888$

3.532384060582640

$-2.558692882290209$

$-0.738097713155046$

0.810800201332668

$-3.465561480567882$

$-1.808533165886656$

1.417076370884340

2.987767720672192

$-4.620540019401575$

4.215888727543661

$-0.557177295993798$

$-0.627620517426609$

$-2.669565281154631$

1.115766698538549

$-3.428408293914368$

2.724246215964337

$-1.580763198186304$

5.155383597522619

0.013158480532061

3.570288043674219

$-4.785609001619214$

1.749384104123576

$-2.990258080670345$

$-0.265462962413865$

$-0.308955901104497$

1.543309364672654

$-2.220763821995915$

3.204343635302958

$-1.503712122713790$

$-3.586421630448618$

0.430051533408703

2.142682067944168

$-5.213796455406342$

$-3.268651719348917$

3.867601718947805

2.135992620502948

$-1.343911288468354$

2.772673735627546

2.800339331826880

0.611614438529278
$-4.311541231305792$ $-4.355559785184588$ $-3.999722437974528$ $-4.226521627361563$

$-3.799101555437234$

$-3.764789999425780$

$-3.462571267540489$

$-3.237520218611013$

$-2.955309034456249$

$-2.329265535747679$

$-2.613583695369335$

$-2.766122318696048$

$-2.468127933932282$

$-1.897981344081337$

$-1.846096801396394$

$-1.780851138850670$

$-1.748339945251161$

$-1.213843690977994$

$-1.524675852932948$

$-0.998047863924146$

$-0.840740398232050$

$-0.826052271044704$

$-0.604014356941967$

$-0.872046517222470$

$-0.275183002536362$

$-0.505283081227493$

0.105633280182371

0.101369464435287

$-0.167745344517018$

0.454703509642506

0.058565477531026

0.845169450226431

0.769685971131601

0.935841624058495

1.048981066728793

0.915697481392092

1.324655088905041

1.499964731701052

1.757204929892611

1.529169903917479

2.653788326925573

5.769606496052202

2.438379092884007 


$\begin{array}{lrrr}\text { Al } & 3.717052823438131 & 0.632053073674893 & 3.243323255866045 \\ \text { Al } & 1.769401542615549 & -1.436571361057490 & 3.123252279311380 \\ \text { Al } & -2.410711881994962 & -1.587056579239910 & 2.545738185784696 \\ \text { Al } & -0.273216064214880 & -3.331202853589402 & 2.889972910918873 \\ \text { Al } & -4.516859221757181 & 0.152862507087297 & 2.047070334855469 \\ \text { Al } & -0.661569601665757 & 2.866256112336689 & 3.990804698495655 \\ \text { Al } & 1.442975174025030 & 0.863379097359607 & 4.595156893549476 \\ \text { Al } & -2.725091688771545 & 0.664083405790363 & 4.101524366624318 \\ \text { Al } & -0.538922380300606 & -1.227339095315251 & 4.563172274078182 \\ \text { Al } & -0.740920985687914 & 1.025030542290933 & 6.082207155088685\end{array}$




\subsection{Al_54}

54

Al $\quad 3.846903071859114$

Al 0.267990697836572

Al 2.105296323987137

Al -2.276158920584832

Al 2.251903057323627

Al -0.556414787618241

Al -3.183761736865180

Al 1.325080855170299

Al -1.455540221713832

Al $\quad 0.427854585428804$

Al 0.213284688275184

Al 4.319056231066941

Al 4.149075182579766

Al 2.122431874589621

Al $\quad-2.975946778218441$

Al 3.395363674270195

Al $\quad-0.408396478084299$

Al -2.252354915929850

Al 1.418460958079585

Al 2.583829544675766

Al -1.244143235771880

Al 0.607686664632972

Al $\quad-6.683426549044088$

Al $\quad-4.994059586941233$

Al 2.274907111714644

Al 0.601795388819795

Al $\quad-3.270310970403359$

Al -1.428894730122513

Al -4.827954044221902

Al 1.531020631723127

Al -0.340627241191111

Al 3.484130055083604

Al 4.190871337161992

Al $\quad-4.717044151817463$

Al 2.797317920973994

Al -1.078293979669153

Al 0.775253162353586

Al $\quad-4.879922943433137$

Al $\quad-2.896263789427342$

Al 0.661069124686424

Al -3.241661130452658

Al -1.417071883602491

Al 3.687404278155736
$-1.951078618296101$ $-2.041540843353296$

0.122315889996429

$-1.598089104787558$

$-4.072193509923145$

0.519578034393612

1.116004001618348

2.519656363237095

3.064158075206056

4.951365455770260

$-4.083780450364181$

$-3.667924077602309$

$-0.147637975606543$

$-1.998857546383134$

$-0.886764326022591$

2.385188322850222

$-1.452165206946878$

$-3.537263511675163$

0.500056756890809

4.849975380241668

1.122679402013929

6.754319837613528

$-1.147093073687916$

1.000391788839709

$-4.068253981318415$

3.012288949957398

3.002700247790987

4.907637056089857

$-1.001461782873195$

$-1.416602731655425$

$-3.515025079844722$

0.590556898715935

$-2.041564012973167$

$-2.998167208650282$

3.125495983224162

$-0.842235199813265$

1.160987638925041

$-0.999787149029649$

$-3.012250674355288$

4.932609762100267

1.184276635947000

3.148434422545209

$-1.336517684052303$
$-4.378042347509639$

$-4.253141873920666$

$-4.182430735454237$

$-3.396995095066855$

$-4.036293932355752$

$-3.338582543741318$

$-2.711392012817772$

$-3.371763372604466$

$-2.709771500140242$

$-2.757611110311263$

$-2.233284880089290$

$-2.314540887160350$

$-2.200683570016963$

$-2.169183191935337$

$-0.588857316606342$

$-1.498688531682545$

$-1.344710451500788$

$-1.382234472325541$

$-1.362191823943245$

$-0.805467955550446$

$-0.548623714096410$

$-0.770723246687778$

$-0.746031085257894$

$-0.556185681844863$

$-0.290467464380734$

$-0.645997071813021$

$-0.461154473115355$

$-0.555687526255220$

$-2.716733568764012$

0.562978692866231

0.549640209912916

0.521857766542643

$-0.111274049499833$

$-0.569892672292919$

1.279323232177266

1.427191152148989

1.396461980786512

1.416597816364142

1.408326912490715

1.331568151623086

1.596050828014512

1.583794501610894

2.435086699667952 


$\begin{array}{lrrr}\text { Al } & -0.923549045494946 & -3.019681543381736 & 3.227154184375822 \\ \text { Al } & 0.735823151270944 & 3.138630781260868 & 3.313517672269978 \\ \text { Al } & -3.030095231423577 & -0.927684431389457 & 3.364583517520158 \\ \text { Al } & 0.957147295426328 & -0.822725988747338 & 3.301504349641242 \\ \text { Al } & 1.706140873519693 & -3.546986505372843 & 2.350556458590940 \\ \text { Al } & -1.264216336492762 & 1.210070745762733 & 3.510699222943860 \\ \text { Al } & -1.090097987250434 & -1.084364159178444 & 5.166510561147019 \\ \text { Al } & 2.977789421778112 & 1.252620339807279 & 3.186873086827909 \\ \text { Al } & 1.259749037422131 & -1.081030005614764 & 6.331651896411017 \\ \text { Al } & 2.702443820039777 & -2.525094163344062 & 4.598166327703350 \\ \text { Al } & 1.059126655869198 & 1.251821775444782 & 5.148542937103935\end{array}$




\subsection{Al_55}

55

Al $\quad 3.934905779360893$

Al 0.485549701030763

Al 2.984282696397269

Al -2.229205196184175

Al -3.262080084231968

Al 0.699666563993356

Al $\quad-0.716159126440626$

Al 1.859585941248795

Al 4.642490424293483

Al 1.109824660180628

Al $\quad-2.444675497954455$

Al $\quad-4.005064448095078$

Al 2.524857101846438

Al -3.391725967034937

Al -1.535382258888053

Al 0.375391028666360

Al 3.666244945325611

Al $\quad 6.190094920721796$

Al 1.655246422405781

Al 2.189588947021418

Al $\quad 5.262445756501714$

Al 1.429073069745620

Al $\quad-4.597863800607556$

Al 4.388948973862103

Al $\quad-0.383119123537044$

Al $\quad-2.294343328367706$

Al -3.6248699748668600

Al -1.126140927241863

Al 0.077728236492888

Al -1.763004969262280

Al 1.718637072457675

Al -2.460445440802975

Al $\quad-4.786915995004590$

Al $\quad-2.945735366491119$

Al -1.327009332723417

Al 2.880692944515355

Al 0.349968758029297

Al -1.733056535270441

Al 0.999038600739809

Al $\quad-2.197372828008907$

Al -1.060621700309801

Al -0.950510101996727

Al -0.513652853374305
0.597980439290851

3.544033104524324

$-1.398671094942120$

3.264799122894329

1.258612096024649

6.663705133418102

$-2.277914513027532$

1.105305856342310

$-2.082048779733134$

$-0.721328372982747$

3.370565211456231

$-1.099084630908543$

4.592805271378740

$-1.564371315722864$

$-0.042189556265836$

3.210947478862392

$-3.915599535695276$

$-2.712132646566671$

0.962125157861353

$-3.503587363894724$

$-0.043721150548812$

2.542605961031333

$-0.808083227571180$

$-1.901678485447004$

1.731692658525375

0.117779543536166

1.320448332406512

5.281000001501834

$-1.464880470878926$

$-1.030898926549813$

5.482396589079972

$-1.257820384780938$

$-0.776563102261122$

1.080051193158475

2.448700672435852

3.046466484035696

0.223969311513212

$-3.832124928533348$

$-4.028078699853318$

$-3.201691184041815$

$-0.805652115306437$

$-2.605464446103412$

$-3.476003564380932$
$-1.175848085658066$

$-2.598609360316672$

$-3.013772034409740$

$-2.003944321153009$

$-0.5699433666741201$

0.956115216168966

4.103318526696153

$-2.808562127471793$

$-1.044598187937712$

$-4.827774363209912$

0.960575659600122

$-2.029680743288345$

1.342251322275853

$-4.643734708725373$

$-5.951970548272983$

0.166879694291138

1.088003590427054

1.107961263917302

0.459449402626355

$-1.125096105933608$

1.097204031146976

2.777146424827453

0.610124966581102

2.967905209393914

$-4.282130284162642$

4.521592407359179

2.256234007491615

$-0.599805513205439$

1.398066077525682

$-0.487463818405441$

$-1.252546665324566$

2.189505941965443

3.579380778743186

$-3.476812843614598$

3.557603726029349

$-1.131104480102863$

3.629630646668321

2.030936819300935

1.571464886112764

$-2.700190393891157$

$-3.200141273265366$

$-5.376359854925058$

$-0.579353779790752$ 


$\begin{array}{lrcc}\text { Al } & -0.570757330153484 & 1.199193984519894 & -1.459091247734047 \\ \text { Al } & 4.036394012900624 & 2.349968791424930 & 1.286738501725916 \\ \text { Al } & -4.606788555316356 & -3.187583239024848 & 2.169049373975543 \\ \text { Al } & 3.032267982380112 & 0.415532974881657 & 2.967397995156974 \\ \text { Al } & -3.306688939956581 & -2.868495918060900 & 4.467187084470224 \\ \text { Al } & 0.906580582973914 & -1.038414381003273 & -1.264296779251540 \\ \text { Al } & -3.376357723384070 & -3.243234630528031 & -0.247904626729898 \\ \text { Al } & 1.823125476584949 & -2.099304590837788 & 3.403255036724147 \\ \text { Al } & 0.617918687560332 & -3.073368605422088 & -3.212091288926269 \\ \text { Al } & 2.734396430501761 & -1.383069037946578 & 0.770995886482332 \\ \text { Al } & -0.974834932559357 & 1.011985516102369 & 1.283506277437917 \\ \text { Al } & -0.390563379673910 & 4.620388012613418 & 2.343346047326131\end{array}$

